\title{
Leading singularities and off-shell conformal integrals
}

\author{
James Drummond, ${ }^{a, b}$ Claude Duhr, ${ }^{c, d}$ Burkhard Eden, ${ }^{e}$ Paul Heslop, ${ }^{f}$ \\ Jeffrey Pennington ${ }^{g}$ and Vladimir A. Smirnov ${ }^{e, h}$ \\ ${ }^{a}$ PH-TH, CERN, Case C01600, CH-1211 Geneva 23, Switzerland \\ ${ }^{b}$ LAPTH, CNRS et Université de Savoie, \\ F-74941 Annecy-le-Vieux Cedex, France \\ ${ }^{c}$ Institut für Theoretische Physik, ETH Zürich, \\ Wolfgang-Pauli-Str. 27, CH-8093, Switzerland \\ ${ }^{d}$ Institute for Particle Physics Phenomenology, University of Durham, \\ Science Laboratories, South Rd., Durham, DH1 3LE, U.K. \\ ${ }^{e}$ Institut für Mathematik, Humboldt-Universität, \\ Zum großen Windkanal 6, 12489 Berlin, Germany \\ ${ }^{f}$ Dept. of Mathematical Sciences, Durham University, \\ Science Laboratories, South Rd., Durham DH1 3LE, U.K. \\ ${ }^{g}$ SLAC National Accelerator Laboratory, Stanford University, Stanford, CA 94309, U.S.A. \\ ${ }^{h}$ Lomonosov Moscow State University, Skobeltsyn Institute of Nuclear Physics, \\ Leninskie gory, Moscow 119992, Russia \\ E-mail: drummond@cern.ch, duhrc@itp.phys.ethz.ch, \\ eden@math.hu-berlin.de, paul.heslop@durham.ac.uk, \\ jpennin@stanford.edu, smirnov@theory.sinp.msu.ru
}

ABSTRACT: The three-loop four-point function of stress-tensor multiplets in $\mathcal{N}=4$ super Yang-Mills theory contains two so far unknown, off-shell, conformal integrals, in addition to the known, ladder-type integrals. In this paper we evaluate the unknown integrals, thus obtaining the three-loop correlation function analytically. The integrals have the generic structure of rational functions multiplied by (multiple) polylogarithms. We use the idea of leading singularities to obtain the rational coefficients, the symbol - with an appropriate ansatz for its structure - as a means of characterising multiple polylogarithms, and the technique of asymptotic expansion of Feynman integrals to obtain the integrals in certain limits. The limiting behaviour uniquely fixes the symbols of the integrals, which we then lift to find the corresponding polylogarithmic functions. The final formulae are numerically confirmed. The techniques we develop can be applied more generally, and we illustrate this by analytically evaluating one of the integrals contributing to the same four-point function at four loops. This example shows a connection between the leading singularities and the entries of the symbol.

KEYwORDS: Supersymmetric gauge theory, Scattering Amplitudes, Extended Supersymmetry

ArXiv EPRINT: 1303.6909

In memory of Francis Dolan. 


\section{Contents}

1 Introduction 2

2 Conformal four-point integrals and single-valued polylogarithms $\quad 8$

2.1 The symbol 8

2.2 Single-Valued Harmonic Polylogarithms (SVHPLs) 10

$\begin{array}{ll}2.3 \text { The } \bar{x} \rightarrow 0 \text { limit of SVHPLs } & 12\end{array}$

3 The short-distance limit $\quad 13$

4 The Easy integral $\quad 16$

$\begin{array}{ll}4.1 \text { Residues of the Easy integral } & 16\end{array}$

$\begin{array}{ll}4.2 \text { The symbol of } E(x, \bar{x}) & 18\end{array}$

$\begin{array}{ll}\text { 4.3 The analytic result for } E(x, \bar{x}) \text { : uplifting from the symbol } & 19\end{array}$

4.4 The analytic result for $E(x, \bar{x})$ : the direct approach 20

$\begin{array}{lll}4.5 & \text { Numerical consistency tests for } E & 21\end{array}$

5 The Hard integral $\quad 21$

5.1 Residues of the Hard integral 21

5.2 The symbols of $H^{(a)}(x, \bar{x})$ and $H^{(b)}(x, \bar{x}) \quad 24$

5.3 The analytic results for $H^{(a)}(x, \bar{x})$ and $H^{(b)}(x, \bar{x}) \quad 25$

$\begin{array}{lll}5.4 & \text { Numerical consistency checks for } H & 29\end{array}$

$\begin{array}{lll}6 & \text { The analytic result for the three-loop correlator } & 29\end{array}$

7 A four-loop example $\quad 30$

$\begin{array}{lll}7.1 & \text { Asymptotic expansions } & 31\end{array}$

7.2 A differential equation 33

$\begin{array}{lll}7.3 \text { An integral solution } & 35\end{array}$

$\begin{array}{lll}7.4 & \text { Expression in terms of multiple polylogarithms } & 39\end{array}$

7.5 Numerical consistency tests for $I^{(4)} \quad 42$

8 Conclusions $\quad 43$

A Asymptotic expansions of the Easy and Hard integrals $\quad 45$

B An integral formula for the Hard integral 46

$\begin{array}{lll}\text { B.1 Limits } & 47\end{array}$

B.2 First non-trivial example (weight three) 48

$\begin{array}{lll}\text { B.3 Weight five example } & 48\end{array}$

B.4 The function $H^{(a)}$ from the Hard integral 49 


\section{Introduction}

The work presented in this paper is motivated by recent progress in planar $\mathcal{N}=4$ super Yang-Mills (SYM) theory in four dimensions, although the methods that we exploit and further develop should be of much wider applicability.

$\mathcal{N}=4$ SYM theory has many striking properties due to its high degree of symmetry; for instance it is conformally invariant, even as a quantum theory [1-8], and the spectrum of anomalous dimensions of composite operators can be found from an integrable system [9-11]. Most strikingly perhaps, it is related to IIB string theory on $\operatorname{AdS}_{5} \times \mathrm{S}^{5}$ by the AdS/CFT correspondence [12-14]. This is a weak/strong coupling duality in which the same physical system is conveniently described by the field theory picture at weak coupling, while the string theory provides a way of capturing its strong coupling regime. The strong coupling limit of scattering amplitudes in the model has been elaborated in ref. [15] from a string perspective. The formulae take the form of vacuum expectation values of polygonal Wilson loops with light-like edges.

This duality between amplitudes and Wilson loops remains true at weak coupling [1619], extending to the finite terms in $\mathcal{N}=4 \mathrm{SYM}$ previously known relations between the infrared divergences of scattering amplitudes and the ultra-violet divergences of (light-like) Wilson lines in QCD [20-25]. Furthermore, it was recently discovered that both sides of this correspondence can be generated from $n$-point correlation functions of stress-tensor multiplets by taking a certain light-cone limit [26-28].

The four-point function of stress-tensor multiplets was intensely studied in the early days of the AdS/CFT duality, in the supergravity approximation [29-32] as well as at weak coupling. The one-loop [33-35] and two-loop [36, 37] corrections are given by conformal ladder integrals.

A Feynman-graph based three-loop result has never become available because of the formidable size and complexity of multi-leg multi-loop computations. Already the two parallel two-loop calculations [36, 37] drew heavily upon superconformal symmetry. However, a formulation on a maximal ('analytic') superspace [38, 39] makes it apparent that the loop corrections to the lowest $x$-space component are given by a product of a certain polynomial with linear combinations of conformal integrals, cf. ref. [40-43]. Then in ref. [44, 45], using a hidden symmetry permuting integration variables and external variables, the problem of finding the three-loop integrand was reduced down to just four unfixed coefficients without any calculation and further down to only one overall coefficient after a little further analysis. This single overall coefficient can then easily be fixed e.g. by comparing to the MHV four-point three-loop amplitude [46] via the correlator/amplitude duality or by requiring the exponentiation of logarithms in a double OPE limit [44]. 
Beyond the known ladder and the 'tennis court', the off-shell three-loop four-point correlator contains two unknown integrals termed 'Easy' and 'Hard' in ref. [44]. In this work we embark on an analytic evaluation of the Easy and Hard integrals postulating that

- the integrals are sums $\sum_{i} R_{i} F_{i}$, where $R_{i}$ are rational functions and $F_{i}$ are pure functions, i.e. $\mathbb{Q}$-linear combinations of logarithms and multiple polylogarithms [47],

- the rational functions $R_{i}$ are given by the so-called leading singularities (i.e. residues of global poles) of the integrals [48],

- the symbol of each $F_{i}$ can be pinned down by appropriate constraints and then integrated to a unique transcendental function.

The principle of uniform transcendentality, innate to the planar $\mathcal{N}=4$ SYM theory, implies that the symbols of all the pure functions are tensors of uniform rank six. Our strategy will be to make an ansatz for the entries that can appear in the symbols of the pure functions and to write down the most general tensor of uniform rank six of this form. We then impose a set of constraints on this general tensor to pin down the symbols of the pure functions. First of all, the tensor needs to satisfy the integrability condition, a criterion for a general tensor to correspond to the symbol of a transcendental function. Next the symmetries of the integrals induce additional constraints, and finally we equate with single variable expansions corresponding to Euclidean coincidence limits. The latter were elaborated for the Easy and Hard integrals in ref. [49, 50] using the method of asymptotic expansion of Feynman integrals [51]. This expansion technique reduces the original higher-point integrals to two-point integrals, albeit with high exponents of the denominator factors and complicated numerators.

To be specific, up to three loops the off-shell four-point correlator is given by [33-37, 44]

$$
G_{4}(1,2,3,4)=G_{4}^{(0)}+\frac{2\left(N_{c}^{2}-1\right)}{\left(4 \pi^{2}\right)^{4}} R(1,2,3,4)\left[a F^{(1)}+a^{2} F^{(2)}+a^{3} F^{(3)}+O\left(a^{4}\right)\right],
$$

Here $N_{c}$ denotes the number of colors and $a$ is the 't Hooft coupling. $G_{4}^{(0)}$ represents the tree-level contribution and $R(1,2,3,4)$ is a universal prefactor, in particular taking into account the different SU(4) flavours which can appear (see ref. [44, 45] for details). Our focus here is on the loop corrections. These can be written in the compact form (exposing the hidden $S_{4+\ell}$ symmetry) as

$$
F^{(\ell)}\left(x_{1}, x_{2}, x_{3}, x_{4}\right)=\frac{x_{12}^{2} x_{13}^{2} x_{14}^{2} x_{23}^{2} x_{24}^{2} x_{34}^{2}}{\ell !\left(\pi^{2}\right)^{\ell}} \int d^{4} x_{5} \ldots d^{4} x_{4+\ell} \hat{f}^{(\ell)}\left(x_{1}, \ldots, x_{4+\ell}\right),
$$

where

$$
\begin{aligned}
& \hat{f}^{(1)}\left(x_{1}, \ldots, x_{5}\right)=\frac{1}{\prod_{1 \leq i<j \leq 5} x_{i j}^{2}}, \\
& \hat{f}^{(2)}\left(x_{1}, \ldots, x_{6}\right)=\frac{\frac{1}{48} x_{12}^{2} x_{34}^{2} x_{56}^{2}+S_{6} \text { permutations }}{\prod_{1 \leq i<j \leq 6} x_{i j}^{2}},
\end{aligned}
$$




$$
\hat{f}^{(3)}\left(x_{1}, \ldots, x_{7}\right)=\frac{\frac{1}{20}\left(x_{12}^{2}\right)^{2}\left(x_{34}^{2} x_{45}^{2} x_{56}^{2} x_{67}^{2} x_{73}^{2}\right)+S_{7} \text { permutations }}{\prod_{1 \leq i<j \leq 7} x_{i j}^{2}} .
$$

Writing out the sum over permutations in the above expressions, these are written as follows

$$
\begin{aligned}
F^{(1)}= & g_{1234}, \\
F^{(2)}= & h_{12 ; 34}+h_{34 ; 12}+h_{23 ; 14}+h_{14 ; 23} \\
& +h_{13 ; 24}+h_{24 ; 13}+\frac{1}{2}\left(x_{12}^{2} x_{34}^{2}+x_{13}^{2} x_{24}^{2}+x_{14}^{2} x_{23}^{2}\right)\left[g_{1234}\right]^{2}, \\
F^{(3)}= & {\left[L_{12 ; 34}+5 \text { perms }\right]+\left[T_{12 ; 34}+11 \text { perms }\right] } \\
& +\left[E_{12 ; 34}+11 \text { perms }\right]+\frac{1}{2}\left[x_{14}^{2} x_{23}^{2} H_{12 ; 34}+11 \text { perms }\right] \\
& +\left[(g \times h)_{12 ; 34}+5 \text { perms }\right],
\end{aligned}
$$

which involve the following integrals:

$$
\begin{aligned}
g_{1234} & =\frac{1}{\pi^{2}} \int \frac{d^{4} x_{5}}{x_{15}^{2} x_{25}^{2} x_{35}^{2} x_{45}^{2}}, \\
h_{12 ; 34} & =\frac{x_{34}^{2}}{\pi^{4}} \int \frac{d^{4} x_{5} d^{4} x_{6}}{\left(x_{15}^{2} x_{35}^{2} x_{45}^{2}\right) x_{56}^{2}\left(x_{26}^{2} x_{36}^{2} x_{46}^{2}\right)} .
\end{aligned}
$$

At three-loop order we encounter

$$
\begin{aligned}
(g \times h)_{12 ; 34} & =\frac{x_{12}^{2} x_{34}^{4}}{\pi^{6}} \int \frac{d^{4} x_{5} d^{4} x_{6} d^{4} x_{7}}{\left(x_{15}^{2} x_{25}^{2} x_{35}^{2} x_{45}^{2}\right)\left(x_{16}^{2} x_{36}^{2} x_{46}^{2}\right)\left(x_{27}^{2} x_{37}^{2} x_{47}^{2}\right) x_{67}^{2}}, \\
L_{12 ; 34} & =\frac{x_{34}^{4}}{\pi^{6}} \int \frac{d^{4} x_{5} d^{4} x_{6} d^{4} x_{7}}{\left(x_{15}^{2} x_{35}^{2} x_{45}^{2}\right) x_{56}^{2}\left(x_{36}^{2} x_{46}^{2}\right) x_{67}^{2}\left(x_{27}^{2} x_{37}^{2} x_{47}^{2}\right)}, \\
T_{12 ; 34} & =\frac{x_{34}^{2}}{\pi^{6}} \int \frac{d^{4} x_{5} d^{4} x_{6} d^{4} x_{7} x_{17}^{2}}{\left(x_{15}^{2} x_{35}^{2}\right)\left(x_{16}^{2} x_{46}^{2}\right)\left(x_{37}^{2} x_{27}^{2} x_{47}^{2}\right) x_{56}^{2} x_{57}^{2} x_{67}^{2}}, \\
E_{12 ; 34} & =\frac{x_{23}^{2} x_{24}^{2}}{\pi^{6}} \int \frac{d^{4} x_{5} d^{4} x_{6} d^{4} x_{7} x_{16}^{2}}{\left(x_{15}^{2} x_{25}^{2} x_{35}^{2}\right) x_{56}^{2}\left(x_{26}^{2} x_{36}^{2} x_{46}^{2}\right) x_{67}^{2}\left(x_{17}^{2} x_{27}^{2} x_{47}^{2}\right)}, \\
H_{12 ; 34} & =\frac{x_{34}^{2}}{\pi^{6}} \int \frac{d^{4} x_{5} d^{4} x_{6} d^{4} x_{7} x_{57}^{2}}{\left(x_{15}^{2} x_{25}^{2} x_{35}^{2} x_{45}^{2}\right) x_{56}^{2}\left(x_{36}^{2} x_{46}^{2}\right) x_{67}^{2}\left(x_{17}^{2} x_{27}^{2} x_{37}^{2} x_{47}^{2}\right)} .
\end{aligned}
$$

Here $g, h, L$ are recognised as the one-loop, two-loop and three-loop ladder integrals, respectively, the dual graphs of the off-shell box, double-box and triple-box integrals. Off-shell, the 'tennis court' integral $T$ can be expressed as the three-loop ladder integral $L$ by using the conformal flip properties ${ }^{1}$ of a two-loop ladder sub-integral [52]. The only new integrals are thus $E$ and $H$ (see figure 1).

Conformal four-point integrals are given by a factor carrying their conformal weight, say, $\left(x_{13}^{2} x_{24}^{2}\right)^{n}$ times some function of the two cross ratios

$$
u=\frac{x_{12}^{2} x_{34}^{2}}{x_{13}^{2} x_{24}^{2}}=x \bar{x}, \quad v=\frac{x_{14}^{2} x_{23}^{2}}{x_{13}^{2} x_{24}^{2}}=(1-x)(1-\bar{x}) .
$$

\footnotetext{
${ }^{1}$ Such identities rely on manifest conformal invariance and will be broken by the introduction of most regulators. For instance, the equivalence of $T$ and $L$ is not true for the dimensionally regulated on-shell integrals.
} 


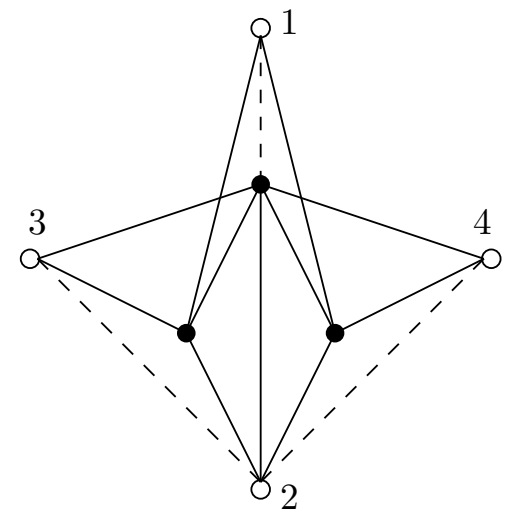

$E_{12 ; 34}$

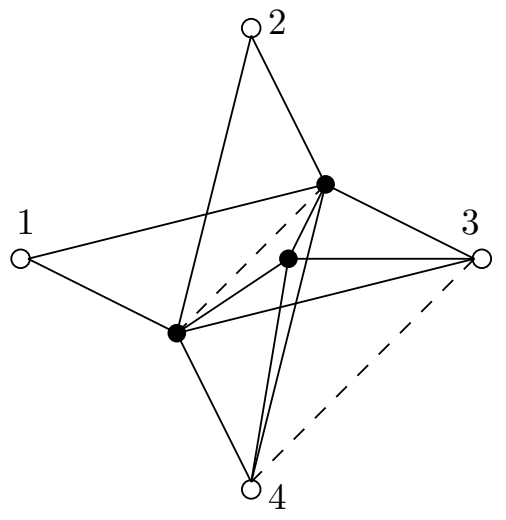

$H_{12 ; 34}$

Figure 1. The Easy and Hard integrals contributing to the correlator of stress tensor multiplets at three loops.

Ladder integrals are explicitly known for any number of loops, see ref. [53, 54] where they are very elegantly expressed as one-parameter integrals. Integration is simplified by the change of variables from the cross-ratios $(u, v)$ to $(x, \bar{x})$ as defined in the last equation. The unique rational prefactor, $x_{13}^{2} x_{24}^{2}(x-\bar{x})$, is common to all cases and can be computed by the leading singularity method as we illustrate shortly. This is multiplied by pure polylogarithm functions which fit with the classification of single-valued harmonic polylogarithms (SVHPLs) in ref. [55]. The associated symbols of the ladder integrals are then tensors composed of the four letters $\{x, \bar{x}, 1-x, 1-\bar{x}\}$.

On the other hand, for generic conformal four-point integrals (of which the Easy and Hard integrals are the first examples) there are no explicit results. Fortunately, in recent years a formalism has been developed in the context of scattering amplitudes to find at least the rational prefactors (i.e. the leading singularities), which are given by the residues of the integrals [48]. There is one leading singularity for each global pole of the integrand and it is obtained by deforming the contour of integration to lie on a maximal torus surrounding the pole in question, i.e. by computing the residue at the global pole. As an illustration, ${ }^{2}$ let us apply this technique to the massive one-loop box integral $g_{1234}$ defined in eq. (1.9). Its leading singularity is obtained by shifting the contour to encircle one of the global poles of the integrand, where all four terms in the denominator vanish. To find this let us consider a change of coordinates from $x_{5}^{\mu}$ to $p_{i}=x_{i 5}^{2}$. The Jacobian for this change of variables is

$$
J=\operatorname{det}\left(\frac{\partial p_{i}}{\partial x_{5}^{\mu}}\right)=\operatorname{det}\left(-2 x_{i 5}^{\mu}\right), \quad J^{2}=\operatorname{det}\left(4 x_{i 5} \cdot x_{j 5}\right)=16 \operatorname{det}\left(x_{i j}^{2}-x_{i 5}^{2}-x_{j 5}^{2}\right),
$$

where the second identity follows by observing that $\operatorname{det}(M)=\sqrt{\operatorname{det}\left(M M^{T}\right)}$. Using this

\footnotetext{
${ }^{2}$ The massless box-integral (i.e. the same integral in the limit $x_{i, i+1}^{2} \rightarrow 0$ ) is discussed in ref. [56] in terms of twistor variables as the simplest example of a 'Schubert problem' in projective geometry. The off-shell case that we discuss here was also recently discussed by S. Caron-Huot (see [57]).
} 
change of variables the massive box becomes

$$
g_{1234}=\frac{1}{\pi^{2}} \int \frac{d^{4} p_{i}}{p_{1} p_{2} p_{3} p_{4} J} .
$$

To find its leading singularity we simply compute the residue around all four poles at $p_{i}=0$ (divided by $2 \pi i$ ). We obtain

$$
g_{1234} \rightarrow \frac{1}{4 \pi^{2} \lambda_{1234}}, \quad \lambda_{1234}=\sqrt{\operatorname{det}\left(x_{i j}^{2}\right)_{i, j=1 . .4}}=x_{13}^{2} x_{24}^{2}(x-\bar{x})
$$

in full agreement with the analytic result $[53,54]$.

Note that we do not consider explicitly a contour around the branch cut associated with the square root factor $\mathrm{J}$ in the denominator of (1.13). Because there is no pole at infinity, the residue theorem guarantees that such a contour is equivalent to the one we already considered. On the other hand, in higher-loop examples, Jacobians from previous integrations cannot be discarded in this manner. In all the examples we consider, these Jacobians always collapse to become simple poles when evaluated on the zero loci of the other denominators and thereby contribute non-trivially to the leading singularity.

The main results of this paper are the analytic evaluations of the Easy and Hard integrals. Due to Jacobian poles, the Easy integral has three distinct leading singularities, out of which only two are algebraically independent, though. The Hard integral has two distinct leading singularities, too. Armed with this information we then attempt to find the pure polylogarithmic functions multiplying these rational factors. Our main inputs for this are analytic expressions for the integrals in the limit $\bar{x} \rightarrow 0$ obtained from the results in [50]. Matching these asymptotic expressions with an ansatz for the symbol of the pure functions we obtain unique answers for the pure functions.

The pure functions contributing to the Easy integral are given by SVHPLs, corresponding to a symbol with entries drawn from the set $\{x, 1-x, \bar{x}, 1-\bar{x}\}$. In this case there is a very straightforward method for obtaining the corresponding function from its asymptotics, by essentially lifting HPLs to SVHPLs as we explain in the next section. However, the SVHPLs are not capable of meeting all constraints for the pure functions contributing to the Hard integral, so that we need to enlarge the set of letters. A natural guess is to include $x-\bar{x}$ (cf. ref. [58]) since it also occurs in the rational factors, and indeed this turns out to be correct. Ultimately, one of the pure functions is found to have a four-letter symbol corresponding to SVHPLs, but the symbol of the other function contains the new letter: the corresponding function cannot be expressed through SVHPLs alone, but it belongs to a more general class of multiple polylogarithms.

Let us stress that the analytic evaluation of the Easy and Hard integrals completes the derivation of the three-loop four-point correlator of stress-tensor multiplets in $\mathcal{N}=4$ SYM. The multiple polylogarithms that we find can be numerically evaluated to very high precision, which paves the way for tests of future integrable system predictions for the four-point function, or for instance for further analyses of the operator product expansion.

Finally, since our set of methods has allowed to obtain the analytic result for the Easy and Hard integrals in a relatively straightforward way (despite the fact that these are 


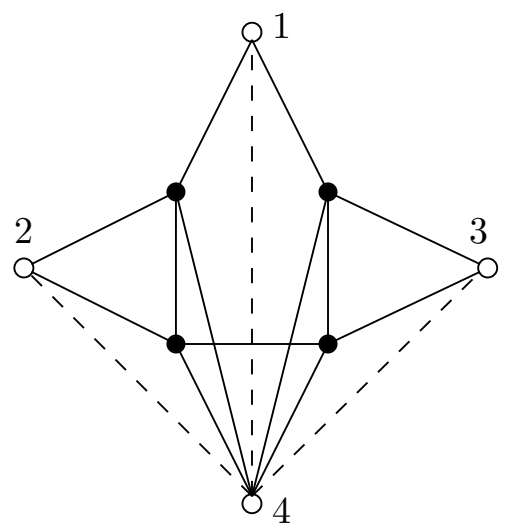

Figure 2. The four-loop integral $I_{14 ; 23}^{(4)}$ defined in eq. (1.15).

not at all simple to evaluate by conventional techniques) we wish to investigate whether this can be repeated to still higher orders. We examine a first relatively simple looking, but non-trivial, four-loop example from the list of integrals contributing to the four-point correlator at that order [45]:

$$
I_{14 ; 23}^{(4)}=\frac{1}{\pi^{8}} \int \frac{d^{4} x_{5} d^{4} x_{6} d^{4} x_{7} d^{4} x_{8} x_{14}^{2} x_{24}^{2} x_{34}^{2}}{x_{15}^{2} x_{18}^{2} x_{25}^{2} x_{26}^{2} x_{37}^{2} x_{38}^{2} x_{45}^{2} x_{46}^{2} x_{47}^{2} x_{48}^{2} x_{56}^{2} x_{67}^{2} x_{78}^{2}} .
$$

The computation of its unique leading singularity follows the same lines as at three loops. However, just as for the Hard integral, the alphabet $\{x, 1-x, \bar{x}, 1-\bar{x}\}$ and the corresponding function space are too restrictive. Interestingly, this integral is related to the Easy integral by a differential equation of Laplace type. Solving this equation promotes the denominator factor $1-u$ of the leading singularities of the Easy integral to a new entry in the symbol of the four-loop integral. Similarly we observe that $(x-\bar{x})$ appears as a leading singularity at one loop and two loops and then appears in the symbol of the Hard integral at three loops, but in this case we do not know any explicit differential equation.

The paper is organised as follows:

- In section 2, we give definitions of the concepts introduced here: symbols, harmonic polylogarithms, SVHPLs, multiple polylogarithms and so on.

- In section 3, we comment on the asymptotic expansion of Feynman integrals.

- In sections 4 and 5 we derive the leading singularities, symbols and ultimately the pure functions corresponding to the Easy and Hard integrals. We also present numerical data indicating the correctness of our results.

- In section 7, we perform a similar calculation for the four-loop integral, $I^{(4)}$.

- Finally we draw some conclusions. We include several appendices collecting some formulae for the asymptotic expansions of the integrals and alternative ways how to derive the analytic results. 


\section{Conformal four-point integrals and single-valued polylogarithms}

The ladder-type integrals that contribute to the correlator are known. More precisely, if we write

$$
\begin{aligned}
g_{13 ; 24} & =\frac{1}{x_{13}^{2} x_{24}^{2}} \Phi^{(1)}(u, v), \\
h_{13 ; 24} & =\frac{1}{x_{13}^{2} x_{24}^{2}} \Phi^{(2)}(u, v), \\
l_{13 ; 24} & =\frac{1}{x_{13}^{2} x_{24}^{2}} \Phi^{(3)}(u, v),
\end{aligned}
$$

then the functions $\Phi^{(L)}(u, v)$ are given by the well-known result [53, 54],

$$
\begin{aligned}
\Phi^{(L)}(u, v)=- & \frac{1}{L !(L-1) !} \int_{0}^{1} \frac{d \xi}{v \xi^{2}+(1-u-v) \xi+u} \log ^{L-1} \xi \\
& \times\left(\log \frac{v}{u}+\log \xi\right)^{L-1}\left(\log \frac{v}{u}+2 \log \xi\right) \\
= & -\frac{1}{x-\bar{x}} f^{(L)}\left(\frac{x}{x-1}, \frac{\bar{x}}{\bar{x}-1}\right),
\end{aligned}
$$

where the conformal cross ratios are given by eq. (1.11) and where we defined the pure function

$$
f^{(L)}(x, \bar{x})=\sum_{r=0}^{L} \frac{(-1)^{r}(2 L-r) !}{r !(L-r) ! L !} \log ^{r}(x \bar{x})\left(\operatorname{Li}_{2 L-r}(x)-\operatorname{Li}_{2 L-r}(\bar{x})\right) .
$$

At this stage, the variables $(x, \bar{x})$ are simply a convenient parametrisation which rationalises the two roots of the quadratic polynomial in the denominator of eq. (2.2). We note that $x$ and $\bar{x}$ are complex conjugate to each other if we work in Euclidean space while they are both real in Minkowski signature.

The particular combination of polylogarithms that appears in eq. (2.2) is not random, but it has a particular mathematical meaning: in Euclidean space, where $x$ and $\bar{x}$ are complex conjugate to each other, the functions $\Phi^{(L)}$ are single-valued functions of the complex variable $x$. In other words, the combination of polylogarithms that appears in the ladder integrals is such that they have no branch cuts in the complex $x$ plane. In order to understand the reason for this, it is useful to look at the symbols of the ladder integrals.

\subsection{The symbol}

One possible way to define the symbol of a transcendental function is to consider its total differential. More precisely, if $F$ is a function whose differential satisfies

$$
d F=\sum_{i} F_{i} d \log R_{i}
$$

where the $R_{i}$ are rational functions, then we can define the symbol of $F$ recursively by [59]

$$
\mathcal{S}(F)=\sum_{i} \mathcal{S}\left(F_{i}\right) \otimes R_{i}
$$


As an example, the symbols of the classical polylogarithms and the ordinary logarithms are given by

$$
\mathcal{S}\left(\operatorname{Li}_{n}(z)\right)=-(1-z) \otimes \underbrace{z \otimes \ldots \otimes z}_{(n-1) \text { times }} \text { and } \mathcal{S}\left(\frac{1}{n !} \ln ^{n} z\right)=\underbrace{z \otimes \ldots \otimes z}_{n \text { times }} .
$$

In addition the symbol satisfies the following identities,

$$
\begin{aligned}
\ldots \otimes(a \cdot b) \otimes \ldots & =\ldots \otimes a \otimes \ldots+\ldots \otimes b \otimes \ldots, \\
\ldots \otimes( \pm 1) \otimes \ldots & =0 \\
\mathcal{S}(F G) & =\mathcal{S}(F) \amalg \mathcal{S}(G),
\end{aligned}
$$

where $\amalg$ denotes the shuffle product on tensors. Furthermore, all multiple zeta values are mapped to zero by the symbol map. Conversely, an arbitrary tensor

$$
\sum_{i_{1}, \ldots, i_{n}} c_{i_{1} \ldots i_{n}} \omega_{i_{1}} \otimes \ldots \otimes \omega_{i_{n}}
$$

whose entries are rational functions is the symbol of a function only if the following integrability condition is fulfilled,

$$
\sum_{i_{1}, \ldots, i_{n}} c_{i_{1} \ldots i_{n}} d \log \omega_{i_{k}} \wedge d \log \omega_{i_{k+1}} \omega_{i_{1}} \otimes \ldots \otimes \omega_{i_{k-1}} \otimes \omega_{i_{k+2}} \otimes \ldots \otimes \omega_{i_{n}}=0
$$

for all consecutive pairs $\left(i_{k}, i_{k+1}\right)$.

The symbol of a function also encodes information about the discontinuities of the function. More precisely, the singularities (i.e. the zeroes or infinities) of the first entries of a symbol determine the branching points of the function, and the symbol of the discontinuity across the branch cut is obtained by dropping this first entry from the symbol. As an example, consider a function $F(x)$ whose symbol has the form

$$
\mathcal{S}(F(x))=\left(a_{1}-x\right) \otimes \ldots \otimes\left(a_{n}-x\right),
$$

where the $a_{i}$ are independent of $x$. Then $F(x)$ has a branching point at $x=a_{1}$, and the symbol of the discontinuity across the branch cut is given by

$$
\mathcal{S}\left[\operatorname{disc}_{a_{1}} F(x)\right]=2 \pi i\left(a_{2}-x\right) \otimes \ldots \otimes\left(a_{n}-x\right) .
$$

If $F$ is a Feynman integral, then the branch cuts of $F$ are dictated by Cutkosky's rules. In particular, for Feynman integrals without internal masses the branch cuts extend between points where one of the Mandelstam invariants becomes zero or infinity. As a consequence, the first entries of the symbol of a Feynman integral must necessarily be Mandelstam invariants [60]. In the case of the four-point position space integrals we are considering in this paper, the first entries of the symbol must then be distances between two points, $x_{i j}^{2}$ for $i, j=1 \ldots 4$. Combined with conformal invariance, this implies that the first entries of the symbols of conformally invariant four-point functions can only be cross ratios. As an example, consider the symbol of the one-loop four-point function,

$$
\mathcal{S}\left[f^{(1)}(x, \bar{x})\right]=u \otimes \frac{1-x}{1-\bar{x}}+v \otimes \frac{\bar{x}}{x}
$$


The first entry condition puts strong constraints on the transcendental functions that can contribute to a conformal four-point function. In order to understand this better let us consider a function whose symbol can be written in the form

$$
\mathcal{S}(F)=u \otimes S_{u}+v \otimes S_{v}=(x \bar{x}) \otimes S_{u}+[(1-x)(1-\bar{x})] \otimes S_{v},
$$

where $S_{u}$ and $S_{v}$ are tensors of lower rank. Let us assume we work in Euclidean space where $x$ and $\bar{x}$ are complex conjugate to each other. It then follows from the previous discussion that $F$ has potential branching points in the complex $x$ plane at $x \in\{0,1, \infty\}$. Let us compute for example the discontinuity of $F$ around $x=0$. Only the first term in eq. (2.13) can give rise to a non-zero contribution, and $x$ and $\bar{x}$ contribute with opposite signs. So we find

$$
\mathcal{S}\left[\operatorname{disc}_{0}(F)\right]=2 \pi i S_{u}-2 \pi i S_{u}=0 .
$$

The argument for the discontinuities around $x=1$ and $x=\infty$ is similar. We thus conclude that $F$ is single-valued in the whole complex $x$ plane. This observation puts strong constraints on the pure functions that might appear in the analytical result for a conformal four-point function. In particular, the ladder integrals $\Phi^{(L)}$ are related to the single-valued analogues of the classical polylogarithms,

$$
D_{n}(x)=\mathfrak{R}_{n} \sum_{k=0}^{n-1} \frac{B_{k} 2^{k}}{k !} \log ^{k}|x| \operatorname{Li}_{n-k}(x),
$$

where $\mathfrak{R}_{n}$ denotes the real part for $n$ odd and the imaginary part otherwise and $B_{k}$ are the Bernoulli numbers. For example, we have

$$
f^{(1)}(x, \bar{x})=4 i D_{2}(x) .
$$

\subsection{Single-Valued Harmonic Polylogarithms (SVHPLs)}

For more general conformal four-point functions more general classes of polylogarithms may appear. The simplest extension of the classical polylogarithms are the so-called harmonic polylogarithms (HPLs), defined by the iterated integrals ${ }^{3}[61]$

$$
H\left(a_{1}, \ldots, a_{n} ; x\right)=\int_{0}^{x} d t f_{a_{1}}(t) H\left(a_{2}, \ldots, a_{n} ; t\right), \quad a_{i} \in\{0,1\}
$$

with

$$
f_{0}(x)=\frac{1}{x} \text { and } f_{1}(x)=\frac{1}{1-x} .
$$

By definition, $H(x)=1$ and in the case where all the $a_{i}$ are zero, we use the special definition

$$
H\left(\overrightarrow{0}_{n} ; x\right)=\frac{1}{n !} \log ^{n} x .
$$

The number $n$ of indices of a harmonic polylogarithm is called its weight. Note that the harmonic polylogarithms contain the classical polylogarithms as special cases,

$$
H\left(\overrightarrow{0}_{n-1}, 1 ; x\right)=\operatorname{Li}_{n}(x) .
$$

\footnotetext{
${ }^{3}$ In the following we use the word harmonic polylogarithm in a restricted sense, and only allow for singularities at $x \in\{0,1\}$ inside the iterated integrals.
} 
In ref. [63] it was shown that infinite classes of generalised ladder integrals can be expressed in terms of single-valued combinations of HPLs. Single-valued analogues of HPLs were studied in detail in ref. [55], and an explicit construction valid for all weights was presented. Here it suffices to say that for every harmonic polylogarithm of the form $H(\vec{a} ; x)$ there is a function $\mathcal{L}_{\vec{a}}(x)$ with essentially the same properties as the ordinary harmonic polylogarithms, but in addition it is single-valued in the whole complex $x$ plane. We will refer to these functions as single-valued harmonic polylogarithms (SVHPLs). Explicitly, the functions $\mathcal{L}_{\vec{a}}(x)$ can be expressed as

$$
\mathcal{L}_{\vec{a}}(x)=\sum_{i, j} c_{i j} H\left(\vec{a}_{i} ; x\right) H\left(\vec{a}_{j} ; \bar{x}\right),
$$

where the coefficients $c_{i j}$ are polynomials of multiple $\zeta$ values such that all branch cuts cancel.

There are two natural symmetry groups acting on the space of SVHPLs. The first symmetry group acts by complex conjugation, i.e., it exchanges $x$ and $\bar{x}$. The conformal four-point functions we are considering are real, and thus eigenfunctions under complex conjugation, while the SVHPLs defined in ref. [55] in general are not. It is therefore convenient to diagonalise the action of this symmetry by defining

$$
\begin{aligned}
L_{\vec{a}}(x) & =\frac{1}{2}\left[\mathcal{L}_{\vec{a}}(x)-(-1)^{|\vec{a}|} \mathcal{L}_{\vec{a}}(\bar{x})\right], \\
\bar{L}_{\vec{a}}(x) & =\frac{1}{2}\left[\mathcal{L}_{\vec{a}}(x)+(-1)^{|\vec{a}|} \mathcal{L}_{\vec{a}}(\bar{x})\right],
\end{aligned}
$$

where $|\vec{a}|$ denotes the weight of $\mathcal{L}_{\vec{a}}(x)$. Note that we have apparently doubled the number of functions, so not all the functions $L_{\vec{a}}(x)$ and $\bar{L}_{\vec{a}}(x)$ can be independent. Indeed, one can observe that

$$
\bar{L}_{\vec{a}}(x)=\left[\text { product of lower weight SVHPLs of the form } L_{\vec{a}}(x)\right] .
$$

The functions $\bar{L}_{\vec{a}}(x)$ can thus always be rewritten as linear combinations of products of SVHPLs of lower weights. In other words, the multiplicative span of the functions $L_{\vec{a}}(x)$ and multiple zeta values spans the whole algebra of SVHPLs. As an example, in this basis the ladder integrals take the very compact form

$$
f^{(L)}(x, \bar{x})=(-1)^{L+1} 2 \underbrace{L_{0, \ldots, 0}, 0,1,0, \ldots, 0}_{L-1}(x) .
$$

While we present most of our result in terms of the $L_{\vec{a}}(x)$, we occasionally find it convenient to employ the $\bar{L}_{\vec{a}}(x)$ and the $\mathcal{L}_{\vec{a}}(x)$ to obtain more compact expressions.

The second symmetry group is the group $S_{3}$ which acts via the transformations of the argument

$$
\begin{array}{lll}
x \rightarrow x, & x \rightarrow 1-x, & x \rightarrow 1 /(1-x), \\
x \rightarrow 1 / x, & x \rightarrow 1-1 / x, & x \rightarrow x /(x-1) .
\end{array}
$$


This action of $S_{3}$ permutes the three singularities $\{0,1, \infty\}$ in the integral representations of the harmonic polylogarithms. In addition, this action has also a physical interpretation. The different cross ratios one can form out of four points $x_{i}$ are parametrised by the group $S_{4} /\left(\mathbb{Z}_{2} \times \mathbb{Z}_{2}\right) \simeq S_{3}$. The action (2.25) is the representation of this group on the cross ratios in the parametrisation (1.11).

\subsection{The $\bar{x} \rightarrow 0$ limit of SVHPLs}

We will be using knowledge of the asymptotic expansions of integrals in the limit $\bar{x} \rightarrow 0$ in order to constrain, and even determine, the integrals themselves. If the function lives in the space of SVHPLs there is a very direct and simple way to obtain the full function from its asymptotic expansion.

This direct procedure relies on the close relation between the series expansion of SVHPLs around $\bar{x}=0$ and ordinary HPLs. In the case where SVHPLs are analytic at $(x, \bar{x})=0$ (i.e. when the corresponding word ends in a ' 1 ') then

$$
\lim _{\bar{x} \rightarrow 0} \mathcal{L}_{w}(x)=H_{w}(x) .
$$

Similar results exist in the case where $\mathcal{L}_{w}(x)$ is not analytic at the origin. In that case the limit does strictly speaking not exist, but we can, nevertheless, represent the function in a neighbourhood of the origin as a polynomial in $\log u$, whose coefficients are analytic functions. More precisely, using the shuffle algebra properties of SVHPLs, we have a unique decomposition

$$
\mathcal{L}_{w}(x)=\sum_{p, w^{\prime}} a_{p, w^{\prime}} \log ^{p} u \mathcal{L}_{w^{\prime}}(x),
$$

where $a_{p, w^{\prime}}$ are integer numbers and $\mathcal{L}_{w^{\prime}}(x)$ are analytic at the origin $(x, \bar{x})=0$.

Conversely, if we are given a function $f(x, \bar{x})$ that around $\bar{x}=0$ admits the asymptotic expansion

$$
f(x, \bar{x})=\sum_{p, w} a_{p, w} \log ^{p} u H_{w}(x)+\mathcal{O}(\bar{x}),
$$

where the $a_{p, w}$ are independent of $(x, \bar{x})$ and $w$ are words made out of the letters 0 and 1 ending in a 1 , there is a unique function $f_{\mathrm{SVHPL}}(x, \bar{x})$ which is a linear combination of products of SVHPLs that has the same asymptotic expansion around $\bar{x}=0$ as $f(x, \bar{x})$. Moreover, this function is simply obtained by replacing the HPLs in eq. (2.28) by their single-valued analogues,

$$
f_{\mathrm{SVHPL}}(x, \bar{x})=\sum_{p, w} a_{p, w} \log ^{p} u \mathcal{L}_{w}(x) .
$$

In other words, $f(x, \bar{x})$ and $f_{\mathrm{SVHPL}}(x, \bar{x})$ agree in the limit $\bar{x} \rightarrow 0$ up to power-suppressed terms.

It is often the case that we find simpler expressions by expanding out all products, i.e. by not explicitly writing the powers of logarithms of $u$. More precisely, replacing $\log u$ by $\log x+\log \bar{x}$ in eq. (2.28) and using the shuffle product for HPLs, we can write eq. (2.28) in the form

$$
f(x, \bar{x})=\sum_{w} a_{w} H_{w}(x)+\log \bar{x} P(x, \log \bar{x})+\mathcal{O}(\bar{x}),
$$


where $P(x, \log \bar{x})$ is a polynomial in $\log \bar{x}$ whose coefficients are HPLs in $x$. From the previous discussion we know that there is a linear combination of SVHPLs that agrees with $f(x, \bar{x})$ up to power-suppressed terms. In fact, this function is independent of the actual form of the polynomial $P$, and is completely determined by the first term in the left-hand side of eq. (2.30),

$$
f_{\mathrm{SVHPL}}(x, \bar{x})=\sum_{w} a_{w} \mathcal{L}_{w}(x) .
$$

So far we have only described how we can always construct a linear combination of SVHPLs that agrees with a given function in the limit $\bar{x} \rightarrow 0$ up to power-suppressed terms. The inverse is obviously not true, and we will encounter such a situation for the Hard integral. In such a case we need to enlarge the space of functions to include more general classes of multiple polylogarithms. Indeed, while SVHPLs have symbols whose entries are all drawn from the set $\{x, \bar{x}, 1-x, 1-\bar{x}\}$, it was observed in ref. [58] that the symbols of three-mass three-point functions (which are related to conformal four-point functions upon sending a point to infinity) in dimensional regularisation involve functions whose symbols also contain the entry $x-\bar{x}$. Function of this type cannot be expressed in terms of HPLs alone, but they require more general classes of multiple polylogarithms, defined recursively by $G(x)=1$ and,

$$
G\left(a_{1}, \ldots, a_{n} ; x\right)=\int_{0}^{x} \frac{d t}{t-a_{1}} G\left(a_{2}, \ldots, a_{n} ; t\right), \quad G\left(\overrightarrow{0}_{p} ; x\right)=\frac{\log ^{p}(x)}{p !},
$$

where $a_{i} \in \mathbb{C}$. We will encounter such functions in later sections when constructing the analytic results for the Easy and Hard integrals.

\section{The short-distance limit}

In this section we sketch how the method of 'asymptotic expansion of Feynman integrals' can deliver asymptotic series for the $\bar{x} \rightarrow 0$ limit of the Easy and the Hard integral. These expansions contain enough information about the integrals to eventually fix ansätze for the full expressions.

In ref. $[49,50]$ asymptotic expansions were derived for both the Easy and Hard integrals in the limits where one of the cross ratios, say $u$, tends to zero. The limit $u \rightarrow 0, v \rightarrow 1$ can be described as a short-distance limit, $x_{2} \rightarrow x_{1}$. Let us assume that we have got rid of the coordinate $x_{4}$ by sending it to infinity and that we are dealing with a function of three coordinates, $x_{1}, x_{2}, x_{3}$, one of which, say $x_{1}$, can be set to zero. The short-distance limit we are interested in then corresponds to $x_{2} \rightarrow 0$, so that the coordinate $x_{2}$ is small (soft) and the coordinate $x_{3}$ is large (hard). This is understood in the Euclidean sense, i.e. $x_{2}$ tends to zero precisely when each of its component tends to zero. One can formalise this by multiplying $x_{2}$ by a parameter $\rho$ and then considering the limit $\rho \rightarrow 0$ upon which $u \sim \rho^{2}, v-1 \sim \rho$.

For a Euclidean limit in momentum space, one can apply the well-known formulae for the corresponding asymptotic expansion written in graph-theoretical language (see ref. [51] for a review). One can also write down similar formulae in position space. In practice, it is often more efficient to apply the prescriptions of the strategy of expansion by regions [51, 64] 
(see also Chapter 9 of ref. [65] for a recent review), which are equivalent to the graphtheoretical prescriptions in the case of Euclidean limits. The situation is even simpler in position space where we work with propagators $1 / x_{i j}^{2}$. It turns out that in order to reveal all the regions contributing to the asymptotic expansion of a position-space Feynman integral it is sufficient to consider each of the integration coordinates $x_{i}$ either soft (i.e. of order $x_{2}$ ) or hard (i.e. of order $x_{3}$ ). Ignoring vanishing contributions, which correspond to integrals without scale, one obtains a set of regions relevant to the given limit. One can reveal this set of regions automatically, using the code described in refs. [66, 67].

The most complicated contributions in the expansion correspond to regions where the internal coordinates are either all hard or soft. For the Easy and Hard integrals, this gives three-loop two-point integrals with numerators. In ref. [49], these integrals were evaluated by treating three numerators as extra propagators with negative exponents, so that the number of the indices in the given family of integrals was increased from nine to twelve. The integrals were then reduced to master integrals using integration-by-parts (IBP) identities using the c++ version of the code FIRE [68]. While this procedure is not optimal, it turned out to be sufficient for the computation in ref. [49]. In ref. [50], a more efficient way was chosen: performing a tensor decomposition and reducing the problem to evaluating integrals with nine indices by the well-known MINCER program [69], which is very fast because it is based on a hand solution of the IBP relations for this specific family of integrals. This strategy has given the possibility to evaluate much more terms of the asymptotic expansion.

It turns out that the expansion we consider includes, within dimensional regularisation, the variable $u$ raised to powers involving an amount proportional to $\epsilon=(4-d) / 2$. A characteristic feature of asymptotic expansions is that individual contributions may exhibit poles. Since the conformal integrals we are dealing with are finite in four dimensions, the poles necessarily cancel, leaving behind some logarithms. The resulting expansions contain powers and logarithms of $u$ times polynomials in $v-1$. Instead of the variable $v$, we turn to the variables $(x, \bar{x})$ defined in eq. (1.11). Note that it is easy to see that in terms of these variables the limit $u \rightarrow 0, v \rightarrow 1$ corresponds to both $x$ and $\bar{x}$ becoming small.

In fact, we only need the leading power term with respect to $u$ and all the terms with respect to $x$. The results of ref. [50] were presented in terms of infinite sums involving harmonic numbers, i.e., for each inequivalent permutation of the external points, it was shown that one can write

$$
I(u, v)=\sum_{k=0}^{3} \log ^{k} u f_{k}(x)+\mathcal{O}(u),
$$

where $I(u, v)$ denotes either the Easy or the Hard integral, and $v=1-x+\mathcal{O}(\bar{x})$. The coefficients $f_{k}(x)$ were expressed as combinations of terms of the form

$$
\sum_{s=1}^{\infty} \frac{x^{s-1}}{s^{i}} S_{\vec{\jmath}}(s) \text { or } \sum_{s=1}^{\infty} \frac{x^{s-1}}{(1+s)^{i}} S_{\vec{\jmath}}(s),
$$

where $S_{\vec{\jmath}}(s)$ are nested harmonic sums [70],

$$
S_{i}(s)=\sum_{n=1}^{s} \frac{1}{n^{i}} \text { and } S_{i \vec{\jmath}}(s)=\sum_{n=1}^{s} \frac{S_{\vec{\jmath}}(n)}{n^{i}} .
$$


To arrive at such explicit results for the coefficients $f_{k}(x)$ a kind of experimental mathematics suggested in ref. [62] was applied: the evaluation of the first terms in the expansion in $x$ gave a hint about the possible dependence of the coefficient at the $n$-th power of $x$. Then an ansatz in the form of a linear combination of nested sums was constructed and the coefficients in this ansatz were fixed by the information about the first terms. Finally, the validity of the ansatz was confirmed using information about the next terms. The complete $x$-expansion was thus inferred from the leading terms.

For the purpose of this paper, it is more convenient to work with polylogarithmic functions in $x$ rather than harmonic sums. Indeed, sums of the type (3.2) can easily be performed in terms of harmonic polylogarithms using the algorithms described in ref. [71]. We note, however, that during the summation process, sums of the type (3.2) with $i=0$ are generated. Sums of this type are strictly speaking not covered by the algorithms of ref. [71], but we can easily reduce them to the case $i \neq 0$ using the following procedure,

$$
\sum_{s=1}^{\infty} x^{s-1} S_{i \vec{\jmath}}(s)=\frac{1}{x} \sum_{s=1}^{\infty} x^{s} \sum_{n=1}^{s} \frac{1}{n_{1}^{i}} S_{\vec{\jmath}}(n)=\frac{1}{x} \sum_{s=0}^{\infty} x^{s} \sum_{n=0}^{s} \frac{1}{n^{i}} S_{\vec{\jmath}}(n),
$$

where the last step follows from $S_{\vec{\jmath}}(0)=0$. Reshuffling the sum by letting $s=n_{1}+n$, we obtain the following relation which is a special case of eq. (96) in ref. [62]:

$$
\sum_{s=1}^{\infty} x^{s-1} S_{i \vec{\jmath}}(s)=\frac{1}{x} \sum_{n_{1}=0}^{\infty} x^{n_{1}} \sum_{n=0}^{\infty} \frac{x^{n}}{n^{i}} S_{\vec{\jmath}}(n)=\frac{1}{1-x} \sum_{s=1}^{\infty} \frac{x^{s-1}}{s^{i}} S_{\vec{\jmath}}(s) .
$$

The last sum is now again of the type (3.2) and can be dealt with using the algorithms of ref. [71].

Performing all the sums that appear in the results of ref. [50], we find for example

$$
\begin{aligned}
x_{13}^{2} x_{24}^{2} E_{14 ; 23}= & \frac{\log u}{x}\left(H_{2,2,1}-H_{2,1,2}+H_{1,3,1}+2 H_{1,2,1,1}-H_{1,1,3}-2 H_{1,1,1,2}\right. \\
& \left.-6 \zeta_{3} H_{2}-6 \zeta_{3} H_{1,1}\right)-\frac{2}{x}\left(2 \zeta_{3} H_{2,1}-4 \zeta_{3} H_{1,2}+4 \zeta_{3} H_{1,1,1}+H_{3,2,1}\right. \\
& -H_{3,1,2}+H_{2,3,1}-H_{2,1,3}+2 H_{1,4,1}+2 H_{1,3,1,1}+2 H_{1,2,2,1}-2 H_{1,1,4} \\
& \left.-2 H_{1,1,2,2}-2 H_{1,1,1,3}-6 \zeta_{3} H_{3}\right)+\mathcal{O}(u) \\
x_{13}^{4} x_{24}^{4} H_{12 ; 34}= & \frac{4 \log u}{x^{2}}\left(H_{1,1,2,1}-H_{1,1,1,2}-6 \zeta_{3} H_{1,1}\right)-\frac{2}{x^{2}}\left(4 H_{2,1,2,1}-4 H_{2,1,1,2}\right. \\
& \left.+4 H_{1,1,3,1}-H_{1,1,2,1,1}-4 H_{1,1,1,3}+H_{1,1,1,2,1}-24 \zeta_{3} H_{2,1}+6 \zeta_{3} H_{1,1,1}\right) \\
& +\mathcal{O}(u),
\end{aligned}
$$

where we used the compressed notation, e.g., $H_{2,1,1,2} \equiv H(0,1,1,1,0,1 ; x)$. The results for the other orientations are rather lengthy, so we do not show them here, but we collect them in appendix A. Let us however comment about the structure of the functions $f_{k}(x)$ that appear in the expansions. The functions $f_{k}(x)$ can always be written in the form

$$
f_{k}(x)=\sum_{l} R_{k, l}(x) \times[\text { HPLs in } x]
$$


where $R_{k, l}(x)$ may represent any of the following rational functions

$$
\frac{1}{x^{2}}, \quad \frac{1}{x}, \quad \frac{1}{x(1-x)} .
$$

We note that the last rational function only enters the asymptotic expansion of $H_{13 ; 24}$.

The aim of this paper is to compute the Easy and Hard integrals by writing for each integral an ansatz of the form

$$
\sum_{i} R_{i}(x, \bar{x}) P_{i}(x, \bar{x})
$$

and to fix the coefficients that appear in the ansatz by matching the limit $\bar{x} \rightarrow 0$ to the asymptotic expansions presented in this section. In the previous section we argued that a natural space of functions for the polylogarithmic part $P_{i}(x, \bar{x})$ are functions that are singlevalued in the complex $x$ plane in Euclidean space. We however still need to determine the rational prefactors $R_{i}(x, \bar{x})$, which are not constrained by single-valuedness.

A natural ansatz would consist in using the same rational prefactors as those appearing in the ladder type integrals. For ladder type integrals we have

$$
R_{i}^{\text {ladder }}(x, \bar{x})=\frac{1}{(x-\bar{x})^{\alpha}}, \quad \alpha \in \mathbb{N},
$$

plus all possible transformations of this function obtained from the action of the $S_{3}$ symmetry (2.25). Then in the limit $u \rightarrow 0$ we obtain

$$
\lim _{\bar{x} \rightarrow 0} R_{i}^{\text {ladder }}(x, \bar{x})=\frac{1}{x^{\alpha}} .
$$

We see that the rational prefactors that appear in the ladder-type integrals can only give rise to rational prefactors in the asymptotic expansions with are pure powers of $x$, and so they can never account for the rational function $1 /(x(1-x))$ that appears in the asymptotic expansion of $H_{13 ; 24}$. We thus need to consider more general prefactors than those appearing in the ladder-type integrals. This issue will be addressed in the next sections.

\section{The Easy integral}

\subsection{Residues of the Easy integral}

The Easy integral is defined as

$$
E_{12 ; 34}=\frac{x_{23}^{2} x_{24}^{2}}{\pi^{6}} \int \frac{d^{4} x_{5} d^{4} x_{6} d^{4} x_{7} x_{16}^{2}}{\left(x_{15}^{2} x_{25}^{2} x_{35}^{2}\right) x_{56}^{2}\left(x_{26}^{2} x_{36}^{2} x_{46}^{2}\right) x_{67}^{2}\left(x_{17}^{2} x_{27}^{2} x_{47}^{2}\right)} .
$$

To find all its leading singularities we order the integrations as follows

$$
E_{12 ; 34}=\frac{x_{23}^{2} x_{24}^{2}}{\pi^{6}}\left[\int \frac{d^{4} x_{6} x_{16}^{2}}{x_{26}^{2} x_{36}^{2} x_{46}^{2}}\left(\int \frac{d^{4} x_{5}}{x_{15}^{2} x_{25}^{2} x_{35}^{2} x_{56}^{2}}\right)\left(\int \frac{d^{4} x_{7}}{x_{17}^{2} x_{27}^{2} x_{47}^{2} x_{67}^{2}}\right)\right] .
$$

First the $x_{7}$ and $x_{5}$ integrations: they are both the same as the massive box computed in the Introduction and thus give leading singularities (see eq. (1.14))

$$
\pm \frac{1}{4 \lambda_{1236}} \quad \pm \frac{1}{4 \lambda_{1246}}
$$


respectively. So we can move directly to the final $x_{6}$ integration

$$
\frac{1}{16 \pi^{6}} \int \frac{d^{4} x_{6} x_{16}^{2}}{x_{26}^{2} x_{36}^{2} x_{46}^{2} \lambda_{1236} \lambda_{1246}} .
$$

Here there are five factors in the denominator and we want to take the residues when four of them vanish to compute the leading singularity, so there are various choices to consider. The simplest option is to cut the three propagators $1 / x_{i 6}^{2}$. Then on this cut we have $\lambda_{1236 \mid \text { cut }}= \pm x_{16}^{2} x_{23}^{2}$ and $\lambda_{1246 \mid \text { cut }}= \pm x_{16}^{2} x_{24}^{2}$, where the vertical line indicates the value on the cut, and the integral reduces to the massive box. This simplification of the $\lambda$ factors is similar to the phenomenon of composite leading singularities [72]. Thus cutting either of the two $\lambda$ s will result in ${ }^{4}$

$$
\text { leading singularity } \# 1 \text { of } E_{12 ; 34}= \pm \frac{1}{64 \pi^{6} \lambda_{1234}} \text {. }
$$

The only other possibility is cutting both $\lambda^{\prime}$ 's. There are then three possibilities, firstly we could cut $x_{26}^{2}$ and $x_{36}^{2}$ as well as the two $\lambda^{\prime} s$. On this cut $\lambda_{1236}$ reduces to $\pm x_{16}^{2} x_{23}^{2}$ and one obtains residue \#1 again. Similarly in the second case where we cut $x_{26}^{2}, x_{46}^{2}$ and the two $\lambda$ s.

So finally we consider the case where we cut $x_{36}^{2}, x_{46}^{2}$ and the two $\lambda$ 's. In this case $\lambda_{1236 \mid \mathrm{cut}}= \pm\left(x_{16}^{2} x_{23}^{2}-x_{13}^{2} x_{26}^{2}\right)$ and $\lambda_{1246 \mid \mathrm{cut}}= \pm\left(x_{16}^{2} x_{24}^{2}-x_{14}^{2} x_{26}^{2}\right)$. Notice that setting $\lambda_{1236}=\lambda_{1246}=0$ means setting $x_{16}^{2}=x_{26}^{2}=0$. We then need to compute the Jacobian associated with cutting $x_{36}^{2}, x_{46}^{2}, \lambda_{1236}, \lambda_{1246}$

$$
\begin{aligned}
\operatorname{det} & \left.\left(\frac{\partial\left(x_{36}^{2}, x_{46}^{2}, \lambda_{1236}, \lambda_{1246}\right)}{\partial x_{6}^{\mu}}\right)\right|_{\text {cut }} \\
& = \pm\left. 16 \operatorname{det}\left(x_{36}^{\mu}, x_{46}^{\mu}, x_{16}^{\mu} x_{23}^{2}-x_{13}^{2} x_{26}^{\mu}, x_{16}^{\mu} x_{24}^{2}-x_{14}^{2} x_{26}^{\mu}\right)\right|_{\text {cut }} \\
& = \pm\left. 16 \operatorname{det}\left(x_{36}^{\mu}, x_{46}^{\mu}, x_{16}^{\mu}, x_{26}^{\mu}\right)\left(x_{23}^{2} x_{14}^{2}-x_{24}^{2} x_{13}^{2}\right)\right|_{\mathrm{cut}} \\
& = \pm 4 \lambda_{1234}\left(x_{23}^{2} x_{14}^{2}-x_{24}^{2} x_{13}^{2}\right),
\end{aligned}
$$

The result of the $x_{6}$ integral (4.4) is

$$
\left.\frac{1}{64 \pi^{6}} \frac{x_{16}^{2}}{x_{26}^{2} \lambda_{1234}\left(x_{23}^{2} x_{14}^{2}-x_{24}^{2} x_{13}^{2}\right)}\right|_{\text {cut }}
$$

At this point there is a subtlety, since on the cut we have simultaneously $x_{16}^{2} x_{23}^{2}-$ $x_{13}^{2} x_{26}^{2}=x_{16}^{2} x_{24}^{2}-x_{14}^{2} x_{26}^{2}=0$, i.e. $x_{16}^{2}=x_{26}^{2}=0$ and so $\frac{x_{16}^{2}}{x_{26}^{2}}$ is undefined. More specifically, the integral depends on whether we take $x_{16}^{2} x_{23}^{2}-x_{13}^{2} x_{26}^{2}=0$ first or $x_{16}^{2} x_{24}^{2}-x_{14}^{2} x_{26}^{2}=0$ first. So we get two possibilities (after multiplying by the external factors $x_{23}^{2} x_{24}^{2}$ in eq. (4.1)):

$$
\begin{aligned}
& \text { leading singularity } \# 2 \text { of } E_{12 ; 34}= \pm \frac{x_{13}^{2} x_{24}^{2}}{64 \pi^{6} \lambda_{1234}\left(x_{23}^{2} x_{14}^{2}-x_{24}^{2} x_{13}^{2}\right)} \\
& \text { leading singularity } \# 3 \text { of } E_{12 ; 34}= \pm \frac{x_{14}^{2} x_{23}^{2}}{64 \pi^{6} \lambda_{1234}\left(x_{23}^{2} x_{14}^{2}-x_{24}^{2} x_{13}^{2}\right)} .
\end{aligned}
$$

\footnotetext{
${ }^{4}$ With a slight abuse of language, in the following we use the word 'cut' to designate that we look at the zeroes of a certain denominator factor.
} 

form $^{5}$

We conclude that the Easy integral takes the 'leading singularity times pure function'

$$
E_{12 ; 34}=\frac{1}{x_{13}^{2} x_{24}^{2}}\left[\frac{E^{(a)}(x, \bar{x})}{x-\bar{x}}+\frac{E^{(b)}(x, \bar{x})}{(x-\bar{x})(v-1)}+\frac{v E^{(c)}(x, \bar{x})}{(x-\bar{x})(v-1)}\right] .
$$

We note that the $x_{3} \leftrightarrow x_{4}$ symmetry relates $E^{(b)}$ and $E^{(c)}$. Furthermore, putting everything over a common denominator it is easy to see that $E^{(a)}$ can be absorbed into the other two functions. We conclude that there is in fact only one independent function, and the Easy integral can be written in terms of a single pure function $E(x, \bar{x})$ as

$$
E_{12 ; 34}=\frac{1}{x_{13}^{2} x_{24}^{2}(x-\bar{x})(v-1)}\left[E(x, \bar{x})+v E\left(\frac{x}{x-1}, \frac{\bar{x}}{\bar{x}-1}\right)\right] .
$$

The function $E(x, \bar{x})$ is antisymmetric under the interchange of $x, \bar{x}$

$$
E(\bar{x}, x)=-E(x, \bar{x})
$$

to ensure that $E_{12 ; 34}$ is a symmetric function of $x, \bar{x}$, but it possesses no other symmetry.

The other two orientations of the Easy integral are then found by permuting various points and are given by

$$
\begin{aligned}
& E_{13 ; 24}=\frac{1}{x_{13}^{2} x_{24}^{2}(x-\bar{x})(u-v)}\left[u E\left(\frac{1}{x}, \frac{1}{\bar{x}}\right)+v E\left(\frac{1}{1-x}, \frac{1}{1-\bar{x}}\right)\right], \\
& E_{14 ; 23}=\frac{1}{x_{13}^{2} x_{24}^{2}(x-\bar{x})(1-u)}\left[E(1-x, 1-\bar{x})+u E\left(1-\frac{1}{x}, 1-\frac{1}{\bar{x}}\right)\right] .
\end{aligned}
$$

It is thus enough to have an expression for $E(x, \bar{x})$ to determine all possible orientations of the Easy integral. The functional form of $E(x, \bar{x})$ will be the purpose of the rest of this section.

\subsection{The symbol of $E(x, \bar{x})$}

In this subsection we determine the symbol of $E(x, \bar{x})$, and in the next section we describe its uplift to a function. This strategy seems over-complicated in the case at hand, because $E(x, \bar{x})$ can in fact directly be obtained in terms of SVHPLs of weight six from its asymptotic expansion using the method described in section 2.3. The two-step derivation (symbol and subsequent uplift) is included mainly for pedagogical purposes because it equally applies to the Hard integral and our four-loop example, where the functions are not writeable in terms of SVHPLs only so that a direct method yet has to be found.

Returning to the Easy integral, we start by writing down the most general tensor of rank six that

- has all its entries drawn from the set $\{x, 1-x, \bar{x}, 1-\bar{x}\}$,

- satisfies the first entry condition, i.e. the first factors in each tensor are either $x \bar{x}$ or $(1-x)(1-\bar{x})$

- is odd under an exchange of $x$ and $\bar{x}$.

\footnotetext{
${ }^{5} \mathrm{~A}$ similar form of the Easy leading singularities, as well as those of the Hard integral discussed in the next section, was independently obtained by S. Caron-Huot.
} 
This results in a tensor that depends on $2 \cdot 4^{5} / 2=1024$ free coefficients (which we assume to be rational numbers). Imposing the integrability condition (2.9) reduces the number of free coefficients to 28 , which is the number of SVHPLs of weight six that are odd under an exchange of $x$ and $\bar{x}$. The remaining free coefficients can be fixed by matching to the limit $u \rightarrow 0, v \rightarrow 1$, or equivalently $\bar{x} \rightarrow 0$.

In order to take the limit, we drop every term in the symbol containing an entry $1-\bar{x}$ and we replace $\bar{x} \rightarrow u / x$, upon which the singularity is hidden in $u$. As a result, every permutation of our ansatz yields a symbol composed of the three letters $\{u, x, 1-x\}$. This tensor can immediately be matched to the symbol of the asymptotic expansion of the Easy integral discussed in section 3. Explicitly, the limits

$$
\begin{aligned}
x_{13}^{2} x_{24}^{2} E_{12 ; 34} \rightarrow & -\frac{1}{x^{2}}\left[\lim _{\bar{x} \rightarrow 0} E(x, \bar{x})+\lim _{\bar{x} \rightarrow 0} E\left(\frac{x}{x-1}, \frac{\bar{x}}{\bar{x}-1}\right)\right] \\
& +\frac{1}{x} \lim _{\bar{x} \rightarrow 0} E\left(\frac{x}{x-1}, \frac{\bar{x}}{\bar{x}-1}\right) \\
x_{13}^{2} x_{24}^{2} E_{13 ; 24} \rightarrow & -\frac{1}{x} \lim _{\bar{x} \rightarrow 0} E\left(\frac{1}{1-x}, \frac{1}{1-\bar{x}}\right) \\
x_{13}^{2} x_{24}^{2} E_{14 ; 23} \rightarrow & \frac{1}{x} \lim _{\bar{x} \rightarrow 0} E(1-x, 1-\bar{x})
\end{aligned}
$$

can be matched with the asymptotic expansions recast as HPLs. All three conditions are consistent with our ansatz; each of them on its own suffices to determine all remaining constants. The resulting symbol is a linear combination of 1024 tensors with entries drawn from the set $\{x, 1-x, \bar{x}, 1-\bar{x}\}$ and with coefficients $\{ \pm 1, \pm 2\}$.

Note that the uniqueness of the uplift procedure for SVHPLs given in section 2.3 implies that each asymptotic limit is sufficient to fix the symbol.

\subsection{The analytic result for $E(x, \bar{x})$ : uplifting from the symbol}

In this section we determine the function $E(x, \bar{x})$ defined in eq. (4.11) starting from its symbol. As the symbol has all its entries drawn from the set $\{x, 1-x, \bar{x}, 1-\bar{x}\}$, the function $E(x, \bar{x})$ can be expressed in terms of the SVHPLs classified in [55]. Additional single-valued terms ${ }^{6}$ proportional to zeta values can be fixed by again appealing to the asymptotic expansion of the integral.

We start by writing down an ansatz for $E(x, \bar{x})$ as a linear combination of weight six of SVHPLs that is odd under exchange of $x$ and $\bar{x}$. Note that we have some freedom w.r.t. the basis for our ansatz. In the following we choose basis elements containing a single factor of the form $L_{\vec{a}}(x)$. This ensures that all the terms are linearly independent.

Next we fix the free coefficients in our ansatz by requiring its symbol to agree with that of $E(x, \bar{x})$ determined in the previous section. As we had started from SVHPLs with the correct symmetries and weight, all coefficients are fixed in a unique way. We arrive at

\footnotetext{
${ }^{6}$ In principle we cannot exclude at this stage more complicated functions of weight less than six multiplied by zeta values.
} 
the following expression for $E(x, \bar{x})$ :

$$
\begin{aligned}
E(x, \bar{x}) & =4 L_{2,4}-4 L_{4,2}-2 L_{1,3,2}+2 L_{2,1,3}-2 L_{3,1,2}+4 L_{3,2,0} \\
& -2 L_{2,2,1,0}+8 L_{3,1,0,0}+2 L_{3,1,1,0}-2 L_{2,1,1,1,0}
\end{aligned}
$$

For clarity, we suppressed the argument of the $L$ functions and we employed the compressed notation for HPLs, e.g., $L_{3,2,1} \equiv L_{0,0,1,0,1,1}(x, \bar{x})$. The asymptotic limits of the last expression correctly reproduce the terms proportional to zeta values in eq. (3.7) and the formulae in appendix A.

\subsection{The analytic result for $E(x, \bar{x})$ : the direct approach}

Here we quickly give the direct method for obtaining $E(x, \bar{x})$ explicitly from its asymptotics via the method outlined in section 2.3.

The asymptotic value of the Easy integral in the permutation $E_{12 ; 34}$ is given in appendix A. Comparing eq. (A.1) with eq. (4.15) and further writing $\log u=\log x+\log \bar{x}$ and expanding out products of functions we find for the asymptotic value of $E(x, \bar{x})$ :

$$
\begin{aligned}
E(x, \bar{x}) & =4 \zeta_{3} H_{2,1}+2 H_{2,4}-2 H_{4,2}+H_{1,2,3}-H_{1,3,2}-2 H_{1,4,0}+H_{2,1,3}-H_{3,1,2} \\
& +2 H_{3,2,0}-H_{1,3,1,0}+H_{2,1,2,0}-2 H_{2,2,0,0}-H_{2,2,1,0}+H_{3,1,1,0}+2 H_{1,2,0,0,0} \\
& +H_{1,2,1,0,0}-H_{2,1,1,0,0}-20 \zeta_{5} H_{1}+8 \zeta_{3} H_{3}+2 \zeta_{3} H_{1,2} \\
& +\log \bar{x} P(x, \log \bar{x})+\mathcal{O}(\bar{x}),
\end{aligned}
$$

where $P$ is a polynomial in $\log \bar{x}$ with coefficients that are HPLs in $x$. From the discussion in section 2.3 we know that there is a unique combination of SVHPLs with this precise asymptotic behavior, and so we find a natural ansatz for $E(x, \bar{x})$,

$$
\begin{aligned}
E(x, \bar{x}) & =4 \zeta_{3} \mathcal{L}_{2,1}+2 \mathcal{L}_{2,4}-2 \mathcal{L}_{4,2}+\mathcal{L}_{1,2,3}-\mathcal{L}_{1,3,2}-2 \mathcal{L}_{1,4,0}+\mathcal{L}_{2,1,3}-\mathcal{L}_{3,1,2}+2 \mathcal{L}_{3,2,0} \\
& -\mathcal{L}_{1,3,1,0}+\mathcal{L}_{2,1,2,0}-2 \mathcal{L}_{2,2,0,0}-\mathcal{L}_{2,2,1,0}+\mathcal{L}_{3,1,1,0}+2 \mathcal{L}_{1,2,0,0,0}+\mathcal{L}_{1,2,1,0,0} \\
& -\mathcal{L}_{2,1,1,0,0}-20 \zeta_{5} \mathcal{L}_{1}+8 \zeta_{3} \mathcal{L}_{3}+2 \zeta_{3} \mathcal{L}_{1,2} .
\end{aligned}
$$

We have lifted this function from its asymptotics in just one limit $\bar{x} \rightarrow 0$ while we also know two other limits of this function given in eq. (3.7) and appendix A. Remarkably, eq. (4.20) is automatically consistent with these two limits, giving a strong indication that it is indeed the right function. Furthermore, eq. (4.18) can then in turn be rewritten in a way that makes the antisymmetry under exchange of $x$ and $\bar{x}$ manifest, and we recover eq. (4.20). Note also that antisymmetry in $x \leftrightarrow \bar{x}$ was not input anywhere, and the fact that the resulting function is indeed antisymmetric is a non-trivial consistency check.

As an aside we also note here that the form of $E(x, \bar{x})$, expressed in the particular basis of SVHPLs we chose to work with, is very simple, having only coefficients \pm 1 or \pm 2 for the polylogarithms of weight six. Indeed other orientations of $E$ have even simpler forms, for instance

$$
\begin{aligned}
E(1 / x, 1 / \bar{x})= & \mathcal{L}_{2,4}-\mathcal{L}_{3,3}-\mathcal{L}_{1,2,3}+\mathcal{L}_{1,3,2}-\mathcal{L}_{1,4,0}-\mathcal{L}_{2,1,3}+\mathcal{L}_{3,1,2}-\mathcal{L}_{4,0,0}+\mathcal{L}_{4,1,0} \\
& +\mathcal{L}_{1,3,0,0}+\mathcal{L}_{1,3,1,0}-\mathcal{L}_{2,1,2,0}+\mathcal{L}_{2,2,1,0}+\mathcal{L}_{3,0,0,0}-\mathcal{L}_{3,1,1,0}-\mathcal{L}_{1,2,1,0,0} \\
& -\mathcal{L}_{2,1,0,0,0}+\mathcal{L}_{2,1,1,0,0}+8 \zeta_{3} \mathcal{L}_{3}-2 \zeta_{3} \mathcal{L}_{1,2}-6 \zeta_{3} \mathcal{L}_{2,0}-4 \zeta_{3} \mathcal{L}_{2,1}
\end{aligned}
$$


with all coefficients of the weight six SVHPLs being \pm 1 , or in the manifestly antisymmetric form with all weight six SVHPLs with coefficient +1

$$
\begin{aligned}
& E(1 / x, 1 / \bar{x})=L_{2,4}+L_{1,3,2}+L_{3,1,2}+L_{4,1,0}+L_{1,3,0,0} \\
& \quad+L_{1,3,1,0}+L_{2,2,1,0}+L_{3,0,0,0}+L_{2,1,1,0,0}+6 \zeta_{3} L_{3}-2 \zeta_{3} L_{2,1} .
\end{aligned}
$$

\subsection{Numerical consistency tests for $E$}

We have determined the analytic result for the Easy integral relying on the knowledge of its residues, symbol and asymptotic expansions. In order to check the correctness of the result, we evaluated $E_{14 ; 23}$ numerically ${ }^{7}$ and compared it to a direct numerical evaluation of the coordinate space integral using FIESTA $[76,77]$.

To be specific, we evaluate the conformally-invariant function $x_{13}^{2} x_{24}^{2} E_{14 ; 23}$. Applying a conformal transformation to send $x_{4}$ to infinity, the integral takes the simplified form,

$$
\lim _{x_{4} \rightarrow \infty} x_{13}^{2} x_{24}^{2} E_{14 ; 23}=\frac{1}{\pi^{6}} \int \frac{d^{4} x_{5} d^{4} x_{6} d^{4} x_{7} x_{13}^{2} x_{16}^{2}}{\left(x_{15}^{2} x_{25}^{2}\right) x_{56}^{2}\left(x_{26}^{2} x_{36}^{2}\right) x_{67}^{2}\left(x_{17}^{2} x_{37}^{2}\right)},
$$

with only 8 propagators. We use the remaining freedom to fix $x_{13}^{2}=1$ so that $u=x_{12}^{2}$ and $v=x_{23}^{2}$. Other numerical values for $x_{13}^{2}$ are possible, of course, but we found that this choice yields relatively stable numerics.

After Feynman parameterisation, the integral is only seven-dimensional and can be evaluated with off-the-shelf software. We generate the integrand with FIESTA and perform the numerical integration with a stand-alone version of CIntegrate. Using the algorithm Divonne, ${ }^{8}$ we obtain roughly five digits of precision after five million function evaluations.

In total, we checked 40 different pairs of values for the cross ratios and we found very good agreement in all cases. A sample of the numerical checks is shown in table 1. Note that $\delta$ denotes the relative error between the analytic result and the number obtained by FIESTA,

$$
\delta=\left|\frac{N_{\text {analytic }}-N_{\text {FIESTA }}}{N_{\text {analytic }}+N_{\text {FIESTA }}}\right| .
$$

\section{The Hard integral}

\subsection{Residues of the Hard integral}

To find all the leading singularities we consider each integration sequentially as follows

$$
H_{12 ; 34}=\frac{x_{34}^{2}}{\pi^{6}}\left\{\int \frac{d^{4} x_{6}}{x_{16}^{2} x_{26}^{2} x_{36}^{2} x_{46}^{2}}\left[\int \frac{d^{4} x_{5} x_{56}^{2}}{x_{15}^{2} x_{25}^{2} x_{35}^{2} x_{45}^{2}}\left(\int \frac{d^{4} x_{7}}{x_{37}^{2} x_{47}^{2} x_{57}^{2} x_{67}^{2}}\right)\right]\right\} .
$$

Let us start with the $x_{7}$ integration,

$$
\int \frac{d^{4} x_{7}}{x_{37}^{2} x_{47}^{2} x_{57}^{2} x_{67}^{2}}
$$

\footnotetext{
${ }^{7}$ All polylogarithms appearing in this paper have been evaluated numerically using the GINAC [73] and HPL $[74,75]$ packages.

${ }^{8}$ Experience shows that Divonne outperforms other algorithms of the Cuba library for problems roughly this size.
} 


\begin{tabular}{|ccccc|}
\hline$u$ & $v$ & Analytic & FIESTA & $\delta$ \\
\hline 0.1 & 0.2 & 82.3552 & 82.3553 & $6.6 \mathrm{e}-7$ \\
0.2 & 0.3 & 57.0467 & 57.0468 & $3.2 \mathrm{e}-8$ \\
0.3 & 0.1 & 90.3540 & 90.3539 & $5.9 \mathrm{e}-8$ \\
0.4 & 0.5 & 37.1108 & 37.1108 & $1.9 \mathrm{e}-8$ \\
0.5 & 0.6 & 31.9626 & 31.9626 & $1.9 \mathrm{e}-8$ \\
0.6 & 0.2 & 54.2881 & 54.2881 & $6.9 \mathrm{e}-8$ \\
0.7 & 0.3 & 42.6519 & 42.6519 & $4.4 \mathrm{e}-8$ \\
0.8 & 0.9 & 23.0199 & 23.0199 & $1.7 \mathrm{e}-8$ \\
0.9 & 0.5 & 30.8195 & 30.8195 & $2.4 \mathrm{e}-8$ \\
\hline
\end{tabular}

Table 1. Numerical comparison of the analytic result for $x_{13}^{2} x_{24}^{2} E_{14 ; 23}$ against FIESTA for several values of the conformal cross ratios.

This is simply the off-shell box considered in section 1, and so its leading singularities are (see eq. (1.14))

$$
\pm \frac{1}{4 \lambda_{3456}}
$$

Next we turn to the $x_{5}$ integration, which now takes the form

$$
\int \frac{d^{4} x_{5} x_{56}^{2}}{x_{15}^{2} x_{25}^{2} x_{35}^{2} x_{45}^{2} \lambda_{3456}} .
$$

There are five factors in the denominator, and we want to cut four of them to compute the leading singularity. The simplest option is to cut the four propagators $1 / x_{i 5}^{2}$. Doing so would yield a new Jacobian factor $1 / \lambda_{1234}$ (exactly as in the previous subsection) and freeze $\lambda_{3456 \mid \text { cut }}= \pm x_{56}^{2} x_{34}^{2}$. This latter factor simply cancels the numerator and we are left with the final $x_{6}$ integration being that of the box in the Introduction. Putting everything together, the leading singularity for this choice is

$$
\text { leading singularity } \# 1 \text { of } H_{12 ; 34}= \pm \frac{1}{64 \pi^{6} \lambda_{1234}^{2}} \text {. }
$$

Returning to the $x_{5}$ integration, eq. (5.4), we must consider the possibility of cutting $\lambda_{3456}$ and three other propagators. Cutting $x_{35}^{2}$ and $x_{45}^{2}$ immediately freezes $\lambda_{3456 \mid \text { cut }}=$ $\pm x_{56}^{2} x_{34}^{2}$ which is canceled by the numerator. Thus it is not possible to cut these two propagators and $\lambda_{3456}$. However, cutting $x_{15}^{2}, x_{25}^{2}, x_{35}^{2}$ and $\lambda_{3456}$ is possible (the only other possibility, i.e. cutting $x_{15}^{2}, x_{25}^{2}, x_{45}^{2}$ and $\lambda_{3456}$, gives the same result by by invariance of the integral under exchange of $x_{3}$ and $x_{4}$ ). Indeed one finds that when $x_{35}^{2}=0$,

$$
\lambda_{3456}= \pm\left(x_{45}^{2} x_{36}^{2}-x_{56}^{2} x_{34}^{2}\right) .
$$

To compute the leading singularity associated with this pole we need to compute the Jacobian

$$
J=\operatorname{det}\left(\frac{\partial\left(x_{15}^{2}, x_{25}^{2}, x_{35}^{2}, \lambda_{3456}\right)}{\partial x_{5}^{\mu}}\right)
$$


As in the box case, it is useful to consider the square of $J$ (on the cut),

$$
J^{2}=16 \operatorname{det}\left(\begin{array}{cc}
x_{i j}^{2} & -2 x_{i} \cdot \partial \lambda_{3456} / \partial x_{5} \\
-2 x_{i} \cdot \partial \lambda_{3456} / \partial x_{5} & \left(\partial \lambda_{3456} / \partial x_{5}\right)^{2}
\end{array}\right) .
$$

The result of the $x_{5}$ integration is then simply

$$
\left.\frac{x_{56}^{2}}{J x_{45}^{2}}\right|_{\text {cut }}=\left.\frac{x_{36}^{2}}{J x_{34}^{2}}\right|_{\text {cut }},
$$

where the second equality follows since $x_{56}^{2}$ and $x_{45}^{2}$ are to be evaluated on the cut (indicated by the vertical line) for which $x_{45}^{2} x_{36}^{2}-x_{56}^{2} x_{34}^{2}=0$. Finally we need to turn to the remaining $x_{6}$ integral. We are simply left with

$$
\left.\frac{1}{16 \pi^{6}} \int \frac{d^{4} x_{6}}{x_{16}^{2} x_{26}^{2} x_{46}^{2} J}\right|_{\text {cut }}
$$

where we note that the $x_{36}^{2}$ propagator term has canceled with the numerator in eq. (5.9). So we have no choice left for the quadruple cut as there are only four poles. In fact on the other cut of the three propagators we find $J_{\mid \text {cut }}=4\left(x_{14}^{2} x_{23}^{2}-x_{13}^{2} x_{24}^{2}\right) x_{36}^{2}$, and so this brings back the propagator $x_{36}^{2}$.

Computing the Jacobian associated with this final integration thus yields the final result for the leading singularity,

$$
\text { leading singularity } \# 2 \text { of } H_{12 ; 34}= \pm \frac{1}{64 \pi^{6}\left(x_{14}^{2} x_{23}^{2}-x_{13}^{2} x_{24}^{2}\right) \lambda_{1234}} \text {. }
$$

We conclude that the Hard integral can be written as these leading singularities times pure functions, i.e. it has the form

$$
H_{12 ; 34}=\frac{1}{x_{13}^{4} x_{24}^{4}}\left[\frac{H^{(a)}(x, \bar{x})}{(x-\bar{x})^{2}}+\frac{H^{(b)}(x, \bar{x})}{(v-1)(x-\bar{x})}\right],
$$

where $H^{(a),(b)}$ are pure polylogarithmic functions. The pure functions must furthermore satisfy the following properties

$$
\begin{array}{llrl}
H^{(a)}(x, \bar{x}) & =H^{(a)}(\bar{x}, x), & H^{(b)}(x, \bar{x})=-H^{(b)}(\bar{x}, x), \\
H^{(a)}(x, \bar{x})=H^{(a)}(x /(x-1), \bar{x} /(\bar{x}-1)), & H^{(b)}(x, \bar{x})=H^{(b)}(x /(x-1), \bar{x} /(\bar{x}-1)),
\end{array}
$$

in order that $H_{12 ; 34}$ be symmetric in $x, \bar{x}$ and under the permutation $x_{1} \leftrightarrow x_{2}$. Furthermore we would expect that $H^{(a)}(x, x)=0$ in order to cancel the pole at $x-\bar{x}$. In fact it will turn out in this section that even without imposing this condition by hand we will arrive at a unique result which nevertheless has this particular property.

By swapping the points around we automatically get

$$
\begin{aligned}
& H_{13 ; 24}=\frac{1}{x_{13}^{4} x_{24}^{4}}\left[\frac{H^{(a)}(1 / x, 1 / \bar{x})}{(x-\bar{x})^{2}}+\frac{H^{(b)}(1 / x, 1 / \bar{x})}{(u-v)(x-\bar{x})}\right], \\
& H_{14 ; 23}=\frac{1}{x_{13}^{4} x_{24}^{4}}\left[\frac{H^{(a)}(1-x, 1-\bar{x})}{(x-\bar{x})^{2}}+\frac{H^{(b)}(1-x, 1-\bar{x})}{(1-u)(x-\bar{x})}\right] .
\end{aligned}
$$




\begin{tabular}{|ccc|}
\hline Weight & Even & Odd \\
\hline 1 & 2 & 0 \\
2 & 3 & 1 \\
3 & 6 & 3 \\
4 & 12 & 9 \\
5 & 28 & 24 \\
6 & 69 & 65 \\
\hline
\end{tabular}

Table 2. Dimensions of the spaces of integrable symbols with entries drawn from the set $\{x, 1-$ $x, \bar{x}, 1-\bar{x}, x-\bar{x}\}$ and split according to the parity under exchange of $x$ and $\bar{x}$.

\subsection{The symbols of $H^{(a)}(x, \bar{x})$ and $H^{(b)}(x, \bar{x})$}

In order to determine the pure functions contributing to the Hard integral, we proceed just like for the Easy integral and first determine the symbol. For the Hard integral we have to start from two ansätze for the symbols $\mathcal{S}\left[H^{(a)}(x, \bar{x})\right]$ and $\mathcal{S}\left[H^{(b)}(x, \bar{x})\right]$. While both pure functions are invariant under the exchange $x_{1} \leftrightarrow x_{2}, \mathcal{S}\left[H^{(a)}\right]$ must be symmetric under the exchange of $x, \bar{x}$ and $\mathcal{S}\left[H^{(b)}\right]$ has to be antisymmetric, cf. eq. (5.13). Going through exactly the same steps as for $E$ we find that the single-variable limits of the symbols cannot be matched against the data from the asymptotic expansions using only entries from the set $\{x, 1-x, \bar{x}, 1-\bar{x}\}$. We thus need to enlarge the ansatz.

Previously, the letter $x-\bar{x} \sim \lambda_{1234}$ has been encountered in ref. $[58,78]$ in a similar context. We therefore consider all possible integrable symbols made from the letters $\{x, 1-x, \bar{x}, 1-\bar{x}, x-\bar{x}\}$ which obey the initial entry condition (2.13). In the case of the Easy integral, the integrability condition only implied that terms depending on both $x$ and $\bar{x}$ come from products of single-variable functions. Here, on the other hand, the condition is more non-trivial since, for example,

$$
\begin{aligned}
d \log \frac{x}{\bar{x}} \wedge d \log (x-\bar{x}) & =d \log x \wedge d \log \bar{x}, \\
d \log \frac{1-x}{1-\bar{x}} \wedge d \log (x-\bar{x}) & =d \log (1-x) \wedge d \log (1-\bar{x}) .
\end{aligned}
$$

We summarise the dimensions of the spaces of such symbols, split according to parity under exchange of $x$ and $\bar{x}$, in table 2 .

Given our ansatz for the symbols of the functions we are looking for, we then match against the twist two asymptotics as described previously. We find a unique solution for the symbols of both $H^{(a)}$ and $H^{(b)}$ compatible with all asymptotic limits. Interestingly, the limit of $H_{13 ; 24}$ leaves one undetermined parameter in $\mathcal{S}\left[H^{(a)}\right]$, which we may fix by appealing to another limit. In the resulting symbols, the letter $x-\bar{x}$ occurs only in the last two entries of $\mathcal{S}\left[H^{(a)}\right]$ while it is absent from $\mathcal{S}\left[H^{(b)}\right]$. Although we did not impose this as a constraint, $\mathcal{S}\left[H^{(a)}\right]$ goes to zero when $x \rightarrow \bar{x}$, which is necessary since the integral cannot have a pole at $x=\bar{x}$. 


\subsection{The analytic results for $H^{(a)}(x, \bar{x})$ and $H^{(b)}(x, \bar{x})$}

In this section we integrate the symbol of the Hard integral to a function, i.e. we determine the full answers for the functions $H^{(a)}(x, \bar{x})$ and $H^{(b)}(x, \bar{x})$ that contribute to the Hard integral $H_{12 ; 34}$.

In the previous section we already argued that the symbol of $H^{(b)}(x, \bar{x})$ has all its entries drawn from the set $\{x, 1-x, \bar{x}, 1-\bar{x}\}$, and so it is reasonable to assume that $H^{(b)}(x, \bar{x})$ can be expressed in terms of SVHPLs only. We may therefore proceed by lifting directly from the asymptotic form as we did in section 4.4 for the Easy integral. By comparing the form of $H_{13 ; 24}$, eq. (5.14), with its asymptotic value (1.14) we can read off the asymptotic form of $H(1 / x, 1 / \bar{x})$. Writing $\log u$ as $\log x+\log \bar{x}$, expanding out all the functions and neglecting $\log \bar{x}$ terms, we can the lift directly to the full function by simply converting HPLs to SVHPLs. In this way we arrive at

$$
\begin{aligned}
H^{(b)}(1 / x, 1 / \bar{x}) & =2 \mathcal{L}_{2,4}-2 \mathcal{L}_{3,3}-2 \mathcal{L}_{1,1,4}-2 \mathcal{L}_{1,4,0}+2 \mathcal{L}_{1,4,1}-2 \mathcal{L}_{2,3,1}+2 \mathcal{L}_{3,1,2} \\
& -2 \mathcal{L}_{4,0,0}+2 \mathcal{L}_{4,1,0}+2 \mathcal{L}_{1,1,1,3}+2 \mathcal{L}_{1,1,3,0}+2 \mathcal{L}_{1,3,0,0}-2 \mathcal{L}_{1,3,1,1} \\
& -2 \mathcal{L}_{2,1,1,2}+2 \mathcal{L}_{2,1,2,1}+2 \mathcal{L}_{3,0,0,0}-2 \mathcal{L}_{3,1,1,0}-2 \mathcal{L}_{1,1,1,2,1}-2 \mathcal{L}_{1,1,2,1,0} \\
& +2 \mathcal{L}_{1,1,2,1,1}-2 \mathcal{L}_{1,2,1,0,0}+2 \mathcal{L}_{1,2,1,1,0}-2 \mathcal{L}_{2,1,0,0,0}+2 \mathcal{L}_{2,1,1,0,0} \\
& +16 \zeta_{3} \mathcal{L}_{3}-16 \zeta_{3} \mathcal{L}_{2,1}
\end{aligned}
$$

Other orientations although still quite simple do not all share the property that they only have coefficients \pm 2 . Using the basis of SVHPLs that makes the parity under exchange of $x$ and $\bar{x}$ explicit, we can write the last equation in the equivalent form

$$
\begin{aligned}
H^{(b)}(x, \bar{x}) & =16 L_{2,4}-16 L_{4,2}-8 L_{1,3,2}-8 L_{1,4,1}+8 L_{2,1,3}-8 L_{2,2,2}+8 L_{2,3,1}-8 L_{3,1,2} \\
& +16 L_{3,2,0}+8 L_{3,2,1}-8 L_{4,1,1}+4 L_{1,2,2,1}-8 L_{1,3,1,1}-4 L_{2,1,1,2}+8 L_{2,1,2,1} \\
& -8 L_{2,2,1,0}-4 L_{2,2,1,1}+8 L_{3,1,1,0}-4 L_{1,1,2,1,1}-24 L_{2,1,1,1,0}
\end{aligned}
$$

Next, we turn to the function $H^{(a)}(x, \bar{x})$. As the symbol of $H^{(a)}(x, \bar{x})$ contains the entry $x-\bar{x}$, it cannot be expressed through SVHPLs only. Single-valued functions whose symbols have entries drawn from the set $\{x, 1-x, \bar{x}, 1-\bar{x}, x-\bar{x}\}$ have been studied up to weight four in ref. [58], and a basis for the corresponding space of functions was constructed. The resulting single-valued functions are combinations of logarithms of $x$ and $\bar{x}$ and multiple polylogarithms $G\left(a_{1}, \ldots, a_{n} ; 1\right)$, with $a_{i} \in\{0,1 / x, 1 / \bar{x}\}$. Note that the harmonic polylogarithms form a subalgebra of this class of functions, because we have, e.g.,

$$
G\left(0, \frac{1}{x}, \frac{1}{x} ; 1\right)=H(0,1,1 ; x) .
$$

This class of single-valued functions thus provides a natural extension of the SVHPLs we have encountered so far. In the following we show how we can integrate the symbol of $H^{(a)}(x, \bar{x})$ in terms of these functions. The basic idea is the same as for the case of the SVHPLs: we would like to write down the most general linear combination of multiple polylogarithms of this type and fix their coefficients by matching to the symbol and the 
asymptotic expansion of $H^{(a)}(x, \bar{x})$. Unlike the SVHPL case, however, some of the steps are technically more involved, and we therefore discuss these points in detail.

Let us denote by $\mathcal{G}$ the algebra generated by $\log x$ and $\log \bar{x}$ and by multiple polylogarithms $G\left(a_{1}, \ldots, a_{n} ; 1\right)$, with $a_{i} \in\{0,1 / x, 1 / \bar{x}\}$, with coefficients that are polynomials in multiple zeta values. Note that without loss of generality we may assume that $a_{n} \neq 0$. In the following we denote by $\mathcal{G}^{ \pm}$the linear subspaces of $\mathcal{G}$ of the functions that are respectively even and odd under an exchange of $x$ and $\bar{x}$. Our first goal will be to construct a basis for the algebra $\mathcal{G}$, as well as for its even and odd subspaces. As we know the generators of the algebra $\mathcal{G}$, we automatically know a basis for the underlying vector space for every weight. It is however often desirable to choose a basis that "recycles" as much as possible information from lower weights, i.e. we would like to choose a basis that explicitly includes all possible products of lower weight basis elements. Such a basis can always easily be constructed: indeed, a theorem by Radford [79] states that every shuffle algebra is isomorphic to the polynomial algebra constructed out of its Lyndon words. In our case, we immediately obtain a basis for $\mathcal{G}$ by taking products of $\log x$ and $\log \bar{x}$ and $G\left(a_{1}, \ldots, a_{n} ; 1\right)$, where $\left(a_{1}, \ldots, a_{n}\right)$ is a Lyndon word in the three letters $\{0,1 / x, 1 / \bar{x}\}$. Next, we can easily construct a basis for the eigenspaces $\mathcal{G}^{ \pm}$by decomposing each (indecomposable) basis function into its even and odd parts. In the following we use the shorthands

$$
G_{m_{1}, \ldots, m_{k}}^{ \pm}\left(x_{1}, \ldots, x_{k}\right)=\frac{1}{2} G(\underbrace{0, \ldots, 0}_{m_{1}-1}, \frac{1}{x_{1}}, \ldots, \underbrace{0, \ldots, 0}_{m_{k}-1}, \frac{1}{x_{k}} ; 1) \pm(x \leftrightarrow \bar{x}) .
$$

In doing so we have seemingly doubled the number of basis functions, and so not all the eigenfunctions corresponding to Lyndon words can be independent. Indeed, we have for example

$$
G_{1,1}^{+}(x, \bar{x})=\frac{1}{2} G_{1}^{+}(x)^{2}-\frac{1}{2} G_{1}^{-}(x)^{2} .
$$

It is easy to check this relation by computing the symbol of both sides of the equation. Similar relations can be obtained without much effort for higher weight functions. The resulting linearly independent set of functions are the desired bases for the eigenspaces. We can now immediately write down the most general linear combination of elements of weight six in $\mathcal{G}^{+}$and determine the coefficients by matching to the symbol of $H^{(a)}(x, \bar{x})$. As we are working with a basis, all the coefficients are fixed uniquely.

At this stage we have determined a function in $\mathcal{G}^{+}$whose symbol matches the symbol of $H^{(a)}(x, \bar{x})$. We have however not yet fixed the terms proportional to zeta values. We start by parametrising these terms by writing down all possible products of zeta values and basis functions in $\mathcal{G}^{+}$. Some of the free parameters can immediately be fixed by requiring the function to vanish for $x=\bar{x}$ and by matching to the asymptotic expansion. Note that our basis makes it particularly easy to compute the leading term in the limit $\bar{x} \rightarrow 0$, because

$$
\lim _{\bar{x} \rightarrow 0} G_{\vec{m}}^{ \pm}(\ldots, \bar{x}, \ldots)=0 .
$$

In other words, the small $u$ limit can easily be approached by dropping all terms which involve (non-trivial) basis functions that depend on $\bar{x}$. The remaining terms only depend 
on $\log \bar{x}$ and harmonic polylogarithms in $x$. However, unlike for SVHPLs, matching to the asymptotic expansions does not fix uniquely the terms proportional to zeta values. The reason for this is that, while in the SVHPL case we could rely on our knowledge of a basis for the single-valued subspace of harmonic polylogarithms, in the present case we have been working with a basis for the full space, and so the function we obtain might still contain non-trivial discontinuities. In the remainder of this section we discuss how on can fix this ambiguity.

In ref. [58] a criterion was given that allows one to determine whether a given function is single-valued. In order to understand the criterion, let us consider the algebra $\overline{\mathcal{G}}$ generated by multiple polylogarithms $G\left(a_{1}, \ldots, a_{n} ; a_{n+1}\right)$, with $a_{i} \in\{0,1 / x, 1 / \bar{x}\}$ and $a_{n+1} \in\{0,1,1 / x, 1 / \bar{x}\}$, with coefficients that are polynomials in multiple zeta values. Note that $\overline{\mathcal{G}}$ contains $\mathcal{G}$ as a subalgebra. The reason to consider the larger algebra $\overline{\mathcal{G}}$ is that $\overline{\mathcal{G}}$ carries a Hopf algebra structure ${ }^{9}$ [80], i.e. $\overline{\mathcal{G}}$ can be equipped with a coproduct $\Delta: \overline{\mathcal{G}} \rightarrow \overline{\mathcal{G}} \otimes \overline{\mathcal{G}}$. Consider now the subspace $\mathcal{G}_{S V}$ of $\overline{\mathcal{G}}$ consisting of single-valued functions. It is easy to see that $\mathcal{G}_{S V}$ is a subalgebra of $\overline{\mathcal{G}}$. However, it is not a sub-Hopf algebra, but rather $\mathcal{G}_{S V}$ is a $\overline{\mathcal{G}}$-comodule, i.e. $\Delta: \mathcal{G}_{S V} \rightarrow \mathcal{G}_{S V} \otimes \overline{\mathcal{G}}$. In other words, when acting with the coproduct on a single-valued function, the first factor in the coproduct must itself be single-valued. As a simple example, we have

$$
\Delta\left(L_{2}\right)=\frac{1}{2} L_{0} \otimes \log \frac{1-x}{1-\bar{x}}+\frac{1}{2} L_{1} \otimes \log \frac{\bar{x}}{x}
$$

Note that this is a natural extension of the first entry condition discussed in section 2. This criterion can now be used to recursively fix the remaining ambiguities to obtain a singlevalued function. In particular, in ref. [58] an explicit basis up to weight four was constructed for $\mathcal{G}_{S V}$. We extended this construction and obtained a complete basis at weight five, and we refer to ref. [58] about the construction of the basis. All the remaining ambiguities can then easily be fixed by requiring that after acting with the coproduct, the first factor can be decomposed into the basis of $\mathcal{G}_{S V}$ up to weight five. We then finally arrive at

$$
\begin{aligned}
H^{(a)}(x, \bar{x}) & =\mathcal{H}(x, \bar{x})-\frac{28}{3} \zeta_{3} L_{1,2}+164 \zeta_{3} L_{2,0}+\frac{136}{3} \zeta_{3} L_{2,1}-\frac{160}{3} L_{3} L_{2,1}-66 L_{0} L_{1,4} \\
& -\frac{148}{3} L_{0} L_{2,3}+\frac{64}{3} L_{2} L_{3,1}+\frac{52}{3} L_{0} L_{3,2}+16 L_{1} L_{3,2}+36 L_{0} L_{4,1}+64 L_{1} L_{4,1} \\
& +\frac{70}{3} L_{0} L_{1,2,2}+24 L_{0} L_{1,3,1}+\frac{26}{3} L_{1} L_{1,3,1}-8 L_{2} L_{2,1,1}+64 L_{0} L_{2,1,2} \\
& -\frac{58}{3} L_{0} L_{2,2,0}-4 L_{0} L_{2,2,1}+\frac{50}{3} L_{1} L_{2,2,1}-12 L_{0} L_{3,1,0}-\frac{88}{3} L_{0} L_{3,1,1} \\
& +18 L_{1} L_{3,1,1}-\frac{32}{3} L_{0} L_{1,1,2,1}-18 L_{0} L_{1,2,1,1}+\frac{166}{3} L_{0} L_{2,1,1,0}-8 L_{0} L_{2,1,1,1} \\
& +328 \zeta_{3} L_{3}+32 L_{3}^{2}-64 L_{2} L_{4} .
\end{aligned}
$$

\footnotetext{
${ }^{9}$ Note that we consider a slightly extended version of the Hopf algebra considered in ref. [80] that allows us to include consistently multiple zeta values of even weight, see ref. [81, 82].
} 
The function $\mathcal{H}(x, \bar{x})$ is a single-valued combination of multiple polylogarithms that cannot be expressed through SVHPLs alone,

$$
\begin{aligned}
& \mathcal{H}(x, \bar{x})=-128 G_{4, \overline{2}}^{+}-512 G_{5, \overline{1}}^{+}-64 G_{3,1, \overline{2}}^{+}+64 G_{3, \overline{1}, 2}^{+}-64 G_{3, \overline{1}, \overline{2}}^{+}-128 G_{3, \overline{2}, \overline{1}}^{+} \\
& +64 G_{4,1, \overline{1}}^{+}-64 G_{4, \overline{1}, 1}^{+}-448 G_{4, \overline{1}, \overline{1}}^{+}+64 G_{2, \overline{1}, 2,1}^{+}+64 G_{2, \overline{1}, \overline{2}, \overline{1}}^{+}+64 G_{2,2,1, \overline{1}}^{+}+64 G_{2,2, \overline{1}, 1}^{+} \\
& -64 G_{2,2, \overline{1}, \overline{1}}^{+}+128 G_{2, \overline{2}, 1,1}^{+}+128 G_{2, \overline{2}, \overline{1}, \overline{1}}^{+}+256 G_{3,1,1, \overline{1}}^{+}+128 G_{3,1, \overline{1}, 1}^{+}-128 G_{3,1, \overline{1}, \overline{1}}^{+} \\
& +192 G_{3, \overline{1}, 1,1}^{+}-64 G_{3, \overline{1}, 1, \overline{1}}^{+}-64 G_{3, \overline{1}, \overline{1}, 1}^{+}+192 G_{3, \overline{1}, \overline{1}, \overline{1}}^{+}+128 H_{2,4}^{+}-128 H_{4,2}^{+} \\
& +\frac{640}{3} H_{2,1,3}^{+}-\frac{64}{3} H_{2,3,1}^{+}-\frac{256}{3} H_{3,1,2}^{+}+64 H_{2,1,1,2}^{+}-64 H_{2,2,1,1}^{+}+64 L_{0} G_{3, \overline{2}}^{+} \\
& +256 L_{0} G_{4, \overline{1}}^{+}+32 L_{0} G_{2,1, \overline{2}}^{+}+64 L_{0} G_{2,2, \overline{1}}^{+}+96 L_{0} G_{3,1, \overline{1}}^{+}+32 L_{0} G_{3, \overline{1}, 1}^{+}+96 L_{0} G_{3, \overline{1}, \overline{1}}^{+} \\
& -64 L_{0} G_{2,1,1, \overline{1}}^{+}+64 L_{0} G_{2, \overline{1}, \overline{1}, \overline{1}}^{+}-32 L_{1} G_{3, \overline{2}}^{+}-128 L_{1} G_{4, \overline{1}}^{+}-16 L_{1} G_{2,1, \overline{2}}^{+} \\
& -32 L_{1} G_{2,2, \overline{1}}^{+}-80 L_{1} G_{3,1, \overline{1}}^{+}-16 L_{1} G_{3, \overline{1}, 1}^{+}-16 L_{1} G_{3, \overline{1}, \overline{1}}^{+}-64 L_{2} G_{2, \overline{1}, \overline{1}}^{-}+64 L_{4} G_{1, \overline{1}}^{-} \\
& +32 L_{2,2} G_{1, \overline{1}}^{-}-\frac{32}{3} H_{2}^{+} H_{2,2}^{+}-64 H_{2}^{+} H_{2,1,1}^{+}-128 H_{2}^{+} H_{4}^{+}-64 H_{1}^{-} L_{0} G_{2, \overline{1}, \overline{1}}^{-} \\
& -32 L_{0}^{2} G_{3, \overline{1}}^{+}-32 L_{0}^{2} G_{2, \overline{1}, \overline{1}}^{+}+32 L_{0}^{2} G_{1,1,1, \overline{1}}^{+}+32 L_{1} L_{0} G_{3, \overline{1}}^{+}+16 L_{1} L_{0} G_{2,1, \overline{1}}^{+} \\
& +16 L_{1} L_{0} G_{2, \overline{1}, \overline{1}}^{+}-\frac{80}{3} H_{1}^{-} L_{0} L_{2,2}-48 H_{1}^{-} L_{0} L_{2,1,1}+12 H_{1}^{-} L_{1} L_{2,2}+16 L_{0}^{2} H_{2,2}^{+} \\
& +32 L_{0}^{2} H_{2,1,1}^{+}-64 H_{1}^{-} L_{4} L_{0}+16 H_{1}^{-} L_{1} L_{4}+64 L_{3} G_{1,1, \overline{1}}^{+}-\frac{640}{3} H_{3}^{-} H_{2,1}^{-} \\
& +64\left(H_{2,1}^{-}\right)^{2}+128\left(H_{3}^{-}\right)^{2}+32 L_{0} L_{2} G_{2, \overline{1}}^{-}-32 L_{0} L_{2} G_{1,1, \overline{1}}^{-}-16 L_{1} L_{2} G_{2, \overline{1}}^{-} \\
& +\frac{16}{3} L_{0} L_{2} H_{2,1}^{-}+16 H_{1}^{-} L_{2} L_{2,1}-\frac{112}{3} H_{2}^{+} L_{0} L_{2,1}-8 H_{2}^{+} L_{1} L_{2,1}-32 H_{3}^{-} L_{0} L_{2} \\
& -48 H_{1}^{-} L_{3} L_{2}+32 H_{2}^{+} L_{0} L_{3}+16 H_{2}^{+} L_{1} L_{3}+32 H_{1}^{-} L_{0}^{2} G_{2, \overline{1}}^{-}-16 H_{1}^{-} L_{1} L_{0} G_{2, \overline{1}}^{-} \\
& +\frac{16}{3} L_{0}^{3} G_{2, \overline{1}}^{+}+\frac{16}{3} L_{0}^{3} G_{1,1, \overline{1}}^{+}-8 L_{1} L_{0}^{2} G_{2, \overline{1}}^{+}-8 L_{1} L_{0}^{2} G_{1,1, \overline{1}}^{+}+\frac{16}{3} H_{1}^{-} L_{0}^{2} H_{2,1}^{-} \\
& -16\left(H_{1}^{-}\right)^{2} L_{0} L_{2,1}-32 H_{1}^{-} H_{3}^{-} L_{0}^{2}+\frac{8}{3}\left(H_{1}^{-}\right)^{2} L_{3} L_{0}-12\left(H_{1}^{-}\right)^{2} L_{1} L_{3}+28 H_{2}^{+} L_{2}^{2} \\
& +\frac{368\left(H_{2}^{+}\right)^{3}}{9}-16 L_{0}^{2} L_{2} G_{1, \overline{1}}^{-}-8 L_{0} L_{1} L_{2} G_{1, \overline{1}}^{-}+\frac{56}{3} H_{1}^{-} H_{2}^{+} L_{0} L_{2}-8 H_{1}^{-} H_{2}^{+} L_{1} L_{2} \\
& +8\left(H_{1}^{-}\right)^{2} L_{2}^{2}+8\left(H_{2}^{+}\right)^{2} L_{0}^{2}+8\left(H_{2}^{+}\right)^{2} L_{0} L_{1}+\frac{28}{3}\left(H_{1}^{-}\right)^{2} H_{2}^{+} L_{0}^{2}-4\left(H_{1}^{-}\right)^{2} H_{2}^{+} L_{0} L_{1} \\
& -96 H_{2}^{-}\left(H_{1}^{-}\right)^{3} L_{0}+\frac{160}{3}\left(H_{1}^{-}\right)^{3} L_{0} L_{2}+\frac{52}{3} H_{1}^{-} L_{0}^{3} L_{2}+4 H_{1}^{-} L_{0} L_{1}^{2} L_{2} \\
& +4 H_{1}^{-} L_{0}^{2} L_{1} L_{2}+H_{2}^{+} L_{0} L_{1}^{3}+\frac{2}{3} H_{2}^{+} L_{0}^{2} L_{1}^{2}-8 H_{2}^{+} L_{0}^{3} L_{1}+\frac{148}{3}\left(H_{1}^{-}\right)^{4} L_{0}^{2} \\
& +\frac{10}{3}\left(H_{1}^{-}\right)^{2} L_{0}^{4}+5\left(H_{1}^{-}\right)^{2} L_{0}^{2} L_{1}^{2}-\frac{10}{3}\left(H_{1}^{-}\right)^{2} L_{0}^{3} L_{1}-128 \zeta_{3} G_{2, \overline{1}}^{+}-128 \zeta_{3} G_{1,1, \overline{1}}^{+} \\
& +\frac{16}{3} \zeta_{3}\left(H_{1}^{-}\right)^{2} L_{0}+24 \zeta_{3}\left(H_{1}^{-}\right)^{2} L_{1}+\frac{64}{3} \zeta_{3} H_{1}^{-} L_{2},
\end{aligned}
$$

where we used the obvious shorthand

$$
H_{\vec{m}}^{ \pm} \equiv \frac{1}{2} H_{\vec{m}}(x) \pm(x \leftrightarrow \bar{x}) .
$$




\begin{tabular}{|ccccc|}
\hline$u$ & $v$ & Analytic & FIESTA & $\delta$ \\
\hline 0.1 & 0.2 & 269.239 & 269.236 & $6.4 \mathrm{e}-6$ \\
0.2 & 0.3 & 136.518 & 136.518 & $1.9 \mathrm{e}-6$ \\
0.3 & 0.1 & 204.231 & 204.230 & $1.3 \mathrm{e}-6$ \\
0.4 & 0.5 & 61.2506 & 61.2505 & $5.0 \mathrm{e}-7$ \\
0.5 & 0.6 & 46.1929 & 46.1928 & $3.5 \mathrm{e}-7$ \\
0.6 & 0.2 & 82.7081 & 82.7080 & $7.4 \mathrm{e}-7$ \\
0.7 & 0.3 & 57.5219 & 57.5219 & $4.7 \mathrm{e}-7$ \\
0.8 & 0.9 & 24.6343 & 24.6343 & $2.0 \mathrm{e}-7$ \\
0.9 & 0.5 & 34.1212 & 34.1212 & $2.6 \mathrm{e}-7$ \\
\hline
\end{tabular}

Table 3. Numerical comparison of the analytic result for $x_{13}^{4} x_{24}^{4} H_{13 ; 24}$ against FIESTA for several values of the conformal cross ratios.

and similarly for $G_{\vec{m}}^{ \pm}$. In addition, for $G_{\vec{m}}^{ \pm}$the position of $\bar{x}$ is indicated by the bars in the indices, e.g.,

$$
G_{1, \overline{2}, \overline{3}}^{ \pm} \equiv G_{1,2,3}^{ \pm}(x, \bar{x}, \bar{x}) .
$$

Note that we have expressed $\mathcal{H}(x, \bar{x})$ entirely using the basis of $\mathcal{G}^{+}$constructed at the beginning of this section. As a consequence, all the terms are linearly independent and there can be no cancellations among different terms.

\subsection{Numerical consistency checks for $H$}

In the previous section we have determined the analytic result for the Hard integral. In order to check that our method indeed produced the correct result for the integral, we have compared our expression numerically against FIESTA. Specifically, we evaluate the conformally-invariant function $x_{13}^{4} x_{24}^{4} H_{13 ; 24}$. Applying a conformal transformation to send $x_{4}$ to infinity, the integral takes the simplified form,

$$
\lim _{x_{4} \rightarrow \infty} x_{13}^{4} x_{24}^{4} H_{13 ; 24}=\frac{1}{\pi^{6}} \int \frac{d^{4} x_{5} d^{4} x_{6} d^{4} x_{7} x_{13}^{4} x_{57}^{2}}{\left(x_{15}^{2} x_{25}^{2} x_{35}^{2}\right) x_{56}^{2}\left(x_{36}^{2}\right) x_{67}^{2}\left(x_{17}^{2} x_{27}^{2} x_{37}^{2}\right)},
$$

with 9 propagators. As we did for $E_{14 ; 23}$, we use the remaining freedom to fix $x_{13}^{2}=1$ so that $u=x_{12}^{2}$ and $v=x_{23}^{2}$, and perform the numerical evaluation using the same setup. We compare at 40 different values, and find excellent agreement in all cases. A small sample of the numerical checks is shown in table 3 .

\section{The analytic result for the three-loop correlator}

In the previous sections we computed the Easy and Hard integrals analytically. Using eq. (1.8), we can therefore immediately write down the analytic answer for the three-loop 
correlator of four stress tensor multiplets. We find

$$
\begin{aligned}
& x_{13}^{2} x_{24}^{2} F_{3}=\frac{6}{x-\bar{x}}\left[f^{(3)}(x)+f^{(3)}\left(1-\frac{1}{x}\right)+f^{(3)}\left(\frac{1}{1-x}\right)\right] \\
& +\frac{2}{(x-\bar{x})^{2}} f^{(1)}(x)\left[v f^{(2)}(x)+f^{(2)}\left(1-\frac{1}{x}\right)+u f^{(2)}\left(\frac{1}{1-x}\right)\right] \\
& +\frac{4}{x-\bar{x}}\left[\frac{1}{v-1} E(x)+\frac{v}{v-1} E\left(\frac{x}{x-1}\right)+\frac{1}{1-u} E(1-x)\right. \\
& \left.+\frac{u}{1-u} E\left(1-\frac{1}{x}\right)+\frac{u}{u-v} E\left(\frac{1}{x}\right)+\frac{v}{u-v} E\left(\frac{1}{1-x}\right)\right] \\
& +\frac{1}{(x-\bar{x})^{2}}\left[(1+v) H^{(a)}(x)+(1+u) H^{(a)}(1-x)+(u+v) H^{(a)}\left(\frac{1}{x}\right)\right] \\
& +\frac{1}{x-\bar{x}}\left[\frac{v+1}{v-1} H^{(b)}(x)+\frac{1+u}{1-u} H^{(b)}(1-x)+\frac{u+v}{u-v} H^{(b)}\left(\frac{1}{x}\right)\right] .
\end{aligned}
$$

The pure functions appearing in the correlator are defined in eqs. (2.3), (4.20), (5.17) and (5.24). For clarity, we suppressed the dependence of the pure functions on $\bar{x}$, i.e. we write $f^{(L)}(x) \equiv f^{(L)}(x, \bar{x})$ and so on. All the pure functions can be expressed in terms of SVHPLs, except for $H^{(a)}$ which contains functions whose symbols involve $x-\bar{x}$ as an entry. We checked that these contributions do not cancel in the sum over all contributions to the correlator.

\section{A four-loop example}

In this section we will discuss a four-loop integral to illustrate how our techniques can be applied at higher loops. The example we consider contributes to the four-loop four-point function of stress-tensor multiplets in $\mathcal{N}=4 \mathrm{SYM}$. Specifically, we consider the Euclidean, conformal, four-loop integral,

$$
I_{14 ; 23}^{(4)}=\frac{1}{\pi^{8}} \int \frac{d^{4} x_{5} d^{4} x_{6} d^{4} x_{7} d^{4} x_{8} x_{14}^{2} x_{24}^{2} x_{34}^{2}}{x_{15}^{2} x_{18}^{2} x_{25}^{2} x_{26}^{2} x_{37}^{2} x_{38}^{2} x_{45}^{2} x_{46}^{2} x_{47}^{2} x_{48}^{2} x_{56}^{2} x_{67}^{2} x_{78}^{2}}=\frac{1}{x_{13}^{2} x_{24}^{2}} f(u, v),
$$

where the cross ratios $u$ and $v$ are defined by eq. (1.11). As we will demonstrate in the following sections, this integral obeys a second-order differential equation whose solution is uniquely specified by imposing single-valued behaviour, similar to the generalised ladders considered in ref. [63].

The four-loop contribution to the stress-tensor four-point function in $\mathcal{N}=4 \mathrm{SYM}$ contains some integrals that do not obviously obey any such differential equations, and with the effort presented here we also wanted to learn to what extent the two-step procedure of deriving symbols and subsequently uplifting them to functions can be repeated for those cases. Our results are encouraging: the main technical obstacle is obtaining sufficient data from the asymptotic expansions; we show that this step is indeed feasible, at least for $I^{(4)}$, and present the results in section 7.1. Ultimately we find it simpler to evaluate $I^{(4)}$ by solving a differential equation, and in this case the asymptotic expansions provide stringent consistency checks. 


\subsection{Asymptotic expansions}

Let us first consider the limits of the four-loop integral (7.1) and its point permutations for $x_{12}, x_{34} \rightarrow 0$. We derive expressions for its asymptotic expansion in the limit where $u \rightarrow 0, v \rightarrow 1$ similar to those for the Easy and Hard integrals obtained in section 3 . The logarithmic terms can be fully determined, while the non-logarithmic part of the expansion requires four-loop IBP techniques that allow us to reach spin 15. This contains enough information to fix the $\zeta_{n} \log ^{0}(u)$ terms (important for beyond-the-symbol contributions) while the purely rational part of the asymptotic series remains partially undetermined. However, our experience with Easy and Hard has shown that each of the three coincidence limits is (almost) sufficient to pin down the various symbols. Inverting the integrals from one orientation to another ties non-logarithmic terms in one expansion to logarithmic ones in another, so that we do in fact command over much more data than it superficially seems. It is also conceivable to take into account more than the lowest order in $u$.

We start by investigating the asymptotic expansion of the integral $I_{14 ; 23}^{(4)}$ whose coincidence limit $x_{12}, x_{34} \rightarrow 0$ diverges as $\log ^{2} u$. There are three contributing regions: while in the first two regions the original integral factors into a product of two two-loop integrals or a one-loop integral and a trivial three-loop integral, the third part corresponds to the fourloop 'hard' region in which the original integral is simply expanded in the small distances. The coefficients of the logarithmically divergent terms in the asymptotic expansion, i.e. the coefficients of $\log ^{2} u$ and $\log u$, can be worked out from the first two regions alone. It is easy to reach high powers in $x$ and we obtain a safe match onto harmonic series of the type (3.2) with $i>1$. Similar to the case of the Easy and Hard integrals discussed in section 3, we can sum up the harmonic sums in terms of HPLs. Note that the absence of harmonic sums with $i=1$ implies the absence of HPLs of the form $H_{1, \ldots}(x)$.

In the hard region, we have explicitly worked out the contribution from spin zero through eight, i.e., up to and including terms of $\mathcal{O}\left(x^{8}\right)$. By what has been said above about the form of the series, this amount of data is sufficient to pin down the terms involving zeta values, while we cannot hope to fix the purely rational part where the dimension of the ansatz is larger than the number of constraints we can obtain. The linear combination displayed below was found from the limit $\bar{x} \rightarrow 0$ of the symbol of the four-loop integral derived in subsequent sections. Its expansion around $x=0$ reproduces the asymptotic expansion of the integral up to $O\left(x^{8}\right)$. We find

$$
\begin{aligned}
& x_{13}^{2} x_{24}^{2} I_{14 ; 23}^{(4)}= \\
& \frac{1}{2 x} \log ^{2} u\left[H_{2,1,3}-H_{2,3,1}+H_{3,1,2}-H_{3,2,1}+2 H_{2,1,1,2}-2 H_{2,2,1,1}+\zeta_{3}\left(6 H_{3}+6 H_{2,1}\right)\right]+ \\
& \frac{1}{x} \log u\left[-4 H_{2,1,4}+4 H_{2,4,1}-3 H_{3,1,3}+3 H_{3,3,1}-3 H_{4,1,2}+3 H_{4,2,1}-4 H_{2,1,1,3}-4 H_{2,1,2,2}\right. \\
& \left.+4 H_{2,2,2,1}+4 H_{2,3,1,1}-2 H_{3,1,1,2}+2 H_{3,2,1,1}+\zeta_{3}\left(-18 H_{4}-8 H_{2,2}-2 H_{3,1}+8 H_{2,1,1}\right)\right]+ \\
& \frac{1}{x}\left[10 H_{2,1,5}+2 H_{2,2,4}-2 H_{2,3,3}-10 H_{2,5,1}+8 H_{3,1,4}-8 H_{3,4,1}+6 H_{4,1,3}-6 H_{4,3,1}\right. \\
& +6 H_{5,1,2}-6 H_{5,2,1}+8 H_{2,1,1,4}+6 H_{2,1,2,3}+8 H_{2,1,3,2}-2 H_{2,1,4,1}+2 H_{2,2,2,2}-4 H_{2,2,3,1} \\
& -4 H_{2,3,1,2}-10 H_{2,3,2,1}-4 H_{2,4,1,1}+4 H_{3,1,1,3}+6 H_{3,1,2,2}-6 H_{3,2,2,1}-4 H_{3,3,1,1}+4 H_{2,1,1,2,2}
\end{aligned}
$$




$$
\begin{aligned}
& -4 H_{2,1,2,2,1}-4 H_{2,2,1,1,2}+4 H_{2,2,2,1,1}+\zeta_{3}\left(36 H_{5}+8 H_{2,3}+12 H_{3,2}-12 H_{4,1}-4 H_{2,1,2}\right. \\
& \left.\left.-16 H_{2,2,1}-8 H_{3,1,1}\right)+\zeta_{5}\left(10 H_{3}+10 H_{2,1}\right)\right]+\mathcal{O}(u) .
\end{aligned}
$$

Next we turn to the asymptotic expansion of the orientation $I_{12 ; 34}^{(4)}$. Here the Euclidean coincidence limit $x_{12}, x_{34} \rightarrow 0$ is finite, and thus the only region we need to analyse is the four-loop hard region, for which we have determined the asymptotic expansion up to and including terms of $\mathcal{O}\left(x^{15}\right)$. Just like for the non-logarithmic part in the asymptotic expansion of $I_{14 ; 23}^{(4)}$, eq. (7.2), we have fixed the terms proportional to zeta values by matching an ansatz in terms of HPLs onto this data, and once again, the terms not containing zeta values are taken from the relevant limit of the symbol. We find

$$
\begin{aligned}
& x_{13}^{2} x_{24}^{2} I_{12 ; 34}^{(4)}= \\
& \frac{1}{x}\left[4 H_{1,3,4}-4 H_{1,5,2}+2 H_{1,1,2,4}-2 H_{1,1,4,2}+2 H_{1,2,1,4}-2 H_{1,2,3,2}+2 H_{1,3,1,3}+2 H_{1,3,3,1}\right. \\
& -2 H_{1,4,1,2}-2 H_{1,5,1,1}+H_{1,1,2,1,3}+H_{1,1,2,3,1}-H_{1,1,3,1,2}-H_{1,1,3,2,1}+H_{1,2,1,1,3}+H_{1,2,1,3,1} \\
& -H_{1,2,2,1,2}+H_{1,2,2,2,1}-2 H_{1,2,3,1,1}+H_{1,3,1,2,1}-H_{1,3,2,1,1}+H_{1,2,1,1,2,1}-H_{1,2,1,2,1,1}+ \\
& \left.\zeta_{3}\left(8 H_{1,1,3}-8 H_{1,2,2}+4 H_{1,1,2,1}-4 H_{1,2,1,1}\right)+70 \zeta_{7} H_{1}\right]+\mathcal{O}(u) .
\end{aligned}
$$

The expansion around $x=0$ of this expression reproduces the asymptotic expansion of the integral up to $\mathcal{O}\left(x^{15}\right)$.

The most complicated integrals appearing in the asymptotic expansion of $I_{14 ; 23}^{(4)}, I_{12 ; 34}^{(4)}$ are four-loop two-point dimensionally regularised (in position space) integrals which belong to the family of integrals contributing to the evaluation of the five-loop contribution to the Konishi anomalous dimension [49],

$$
\begin{aligned}
G\left(a_{1}, \ldots, a_{14}\right)=\int & \frac{d^{d} x_{6} d^{d} x_{7} d^{d} x_{8} d^{d} x_{9}}{\left(x_{16}^{2}\right)^{a_{1}}\left(x_{17}^{2}\right)^{a_{2}}\left(x_{18}^{2}\right)^{a_{3}}\left(x_{19}^{2}\right)^{a_{4}}\left(x_{6}^{2}\right)^{a_{5}}\left(x_{7}^{2}\right)^{a_{6}}\left(x_{8}^{2}\right)^{a_{7}}} \\
& \times \frac{1}{\left(x_{9}^{2}\right)^{a_{8}}\left(x_{67}^{2}\right)^{a_{9}}\left(x_{68}^{2}\right)^{a_{10}}\left(x_{69}^{2}\right)^{a_{11}}\left(x_{78}^{2}\right)^{a_{12}}\left(x_{79}^{2}\right)^{a_{13}}\left(x_{89}^{2}\right)^{a_{14}}},
\end{aligned}
$$

with various integer indices $a_{1}, \ldots, a_{14}$ and $d=4-2 \epsilon$.

The complexity of the IBP reduction to master integrals is determined, in a first approximation, by the number of positive indices and the maximal deviation from the corner point of a sector, which has indices equal to 0 or 1 for non-positive and positive indices, correspondingly. This deviation can be characterised by the number $\sum_{i \in \nu_{+}}\left(a_{i}-\right.$ 1) $-\sum_{i \in \nu_{-}} a_{i}$ where $\nu_{ \pm}$are sets of positive (negative) indices. So the most complicated (for an IBP reduction) integrals appearing in the contribution of spin $s$ to the asymptotic expansion in the short-distance limit have nine positive indices and the deviation from the corner point is equal to $2 s-2$. It was possible to get results up to spin 15 .

As in ref. [49] the IBP reduction was performed by the c++ version of the code FIRE [68]. The master integrals of this family either reduce, via a dual transformation, to the corresponding momentum space master integrals $[83,84]$ or can be taken from ref. [49]. To arrive at contributions corresponding to higher spin values, FIRE was combined with a recently developed alternative code to solve IBP relations LiteRed [85] based on the algebraic properties of IBP relations revealed in ref. [86]. (See ref. [87] where this combination was presented within the Mathematica version of FIRE.) 


\subsection{A differential equation}

We can use the magic identity [52] on the two-loop ladder subintegral

$$
I^{(2)}\left(x_{1}, x_{2}, x_{4}, x_{7}\right)=h_{17 ; 24}=\frac{1}{\pi^{4}} \int \frac{d^{4} x_{5} d^{4} x_{6} x_{24}^{2}}{x_{15}^{2} x_{25}^{2} x_{26}^{2} x_{45}^{2} x_{46}^{2} x_{67}^{2} x_{56}^{2}} .
$$

The magic identity reads

$$
I^{(2)}\left(x_{1}, x_{2}, x_{4}, x_{7}\right)=I^{(2)}\left(x_{2}, x_{1}, x_{7}, x_{4}\right),
$$

and using it on the four-loop integral we find

$$
\begin{aligned}
I_{14 ; 23}^{(4)} & =\frac{1}{\pi^{4}} \int \frac{d^{4} x_{7} d^{4} x_{8} x_{14}^{2} x_{34}^{2}}{x_{18}^{2} x_{37}^{2} x_{38}^{2} x_{47}^{2} x_{48}^{2} x_{78}^{2}} I^{(2)}\left(x_{1}, x_{2}, x_{4}, x_{7}\right) \\
& =\frac{1}{\pi^{4}} \int \frac{d^{4} x_{7} d^{4} x_{8} x_{14}^{2} x_{34}^{2}}{x_{18}^{2} x_{37}^{2} x_{38}^{2} x_{47}^{2} x_{48}^{2} x_{78}^{2}} I^{(2)}\left(x_{2}, x_{1}, x_{7}, x_{4}\right) \\
& =\frac{1}{\pi^{8}} \int \frac{d^{4} x_{5} d^{4} x_{6} d^{4} x_{7} d^{4} x_{8} x_{17}^{2} x_{14}^{2} x_{34}^{2}}{x_{18}^{2} x_{37}^{2} x_{38}^{2} x_{47}^{2} x_{48}^{2} x_{78}^{2} x_{25}^{2} x_{15}^{2} x_{16}^{2} x_{75}^{2} x_{76}^{2} x_{64}^{2} x_{56}^{2}} .
\end{aligned}
$$

The resulting integral (7.7) is 'boxable', i.e. we may apply the Laplace operator at the point $x_{2}$. The only propagator which depends on $x_{2}$ is the one connected to the point $x_{5}$ and we have

$$
\square_{2} \frac{1}{x_{25}^{2}}=-4 \pi^{2} \delta^{4}\left(x_{25}\right)
$$

The effect of the Laplace operator is therefore to reduce the loop order by one [52]. Thus on the full integral $I^{(4)}$ we have

$$
\begin{aligned}
\square_{2} I_{14 ; 23}^{(4)} & =-\frac{4}{\pi^{6}} \int \frac{d^{4} x_{6} d^{4} x_{7} d^{4} x_{8} x_{17}^{2} x_{14}^{2} x_{34}^{2}}{x_{18}^{2} x_{37}^{2} x_{38}^{2} x_{47}^{2} x_{48}^{2} x_{78}^{2} x_{12}^{2} x_{16}^{2} x_{72}^{2} x_{76}^{2} x_{64}^{2} x_{26}^{2}} \\
& =-4 \frac{x_{14}^{2}}{x_{12}^{2} x_{24}^{2}} E_{14 ; 23},
\end{aligned}
$$

where we have recognised the Easy integral,

$$
E_{14 ; 23}=\frac{1}{\pi^{6}} \int \frac{d^{4} x_{6} d^{4} x_{7} d^{4} x_{8} x_{34}^{2} x_{24}^{2} x_{17}^{2}}{x_{18}^{2} x_{37}^{2} x_{38}^{2} x_{47}^{2} x_{48}^{2} x_{78}^{2} x_{16}^{2} x_{72}^{2} x_{76}^{2} x_{64}^{2} x_{26}^{2}}=\frac{1}{x_{13}^{2} x_{24}^{2}} f_{E}(u, v) .
$$

The differential equation (7.9) becomes an equation for the function $f$,

$$
\square_{2} \frac{1}{x_{13}^{2} x_{24}^{2}} f(u, v)=-4 \frac{x_{14}^{2}}{x_{12}^{2} x_{13}^{2} x_{24}^{4}} f_{E}(u, v) .
$$

Applying the chain rule we obtain the following equation in terms of $u$ and $v$,

$$
\Delta^{(2)} f(u, v)=-\frac{4}{u} f_{E}(u, v),
$$

where

$$
\Delta^{(2)}=4\left[2\left(\partial_{u}+\partial_{v}\right)+u \partial_{u}^{2}+v \partial_{v}^{2}-(1-u-v) \partial_{u} \partial_{v}\right] .
$$

In terms of $(x, \bar{x})$ we have

$$
x \bar{x} \partial_{x} \partial_{\bar{x}} \hat{f}(x, \bar{x})=-\hat{f}_{E}(x, \bar{x}),
$$


where

$$
\hat{f}(x, \bar{x})=-(x-\bar{x}) f(u, v)
$$

and similarly for $\hat{f}_{E}$. Note that $\hat{f}(x, \bar{x})=-\hat{f}(\bar{x}, x)$. Now we recall that the function $f_{E}(u, v)$ defined by eq. (7.10) in the orientation $E_{14 ; 23}$ is of the form

$$
f_{E}(u, v)=\frac{1}{(x-\bar{x})(1-x \bar{x})}\left[E(1-x, 1-\bar{x})+x \bar{x} E\left(1-\frac{1}{x}, 1-\frac{1}{\bar{x}}\right)\right] .
$$

Hence we find the following equation for $\hat{f}$,

$$
(1-x \bar{x}) x \bar{x} \partial_{x} \partial_{\bar{x}} \hat{f}(x, \bar{x})=-\left[E(1-x, 1-\bar{x})+x \bar{x} E\left(1-\frac{1}{x}, 1-\frac{1}{\bar{x}}\right)\right] .
$$

Without examining the equation in great detail we can immediately make the following observations about $\hat{f}$.

- The function $\hat{f}$ is a pure function of weight eight. From eq. (7.15) the only leading singularity of the four-loop integral $I^{(4)}$ is therefore of the $1 /(x-\bar{x})$ type, just as for the ladders.

- The final entries of the symbol of $\hat{f}(x, \bar{x})$ can be written as functions only of $x$ or of $\bar{x}$, but not both together. This follows because the right-hand side of eq. (7.17) contains only functions of weight six, whereas there would be a contribution of weight seven if the final entries could not be separated into functions of $x$ or $\bar{x}$ separately. Indeed the final entries can only be $x$ or $\bar{x}$ themselves due to the form of the prefactor on the 1.h.s. of (7.17).

- The factor $(1-x \bar{x})$ on the left-hand side implies that the next-to-final entries in the symbol of $\hat{f}(x, \bar{x})$ contain the letter $(1-x \bar{x})$. This can be seen from the fact that the r.h.s. of equation (7.17) has no overall factor of $(1-x \bar{x})$. Therefore the prefactor $(1-$ $x \bar{x})$ on the 1.h.s. of (7.17) must be cancelled by a denominator obtained by the action of the differential operator on the symbol entries of $\hat{f}$. Since the final entries are only $x$ or of $\bar{x}$, the factor $(1-x \bar{x})$ must appear in some of the penultimate slots of the symbol.

In ref. [63], slightly simpler, but very similar, equations were analysed for a class of generalised ladder integrals. The analysis of ref. [63] can be adapted to the case of the four-loop integral $I^{(4)}$ and, as in ref. [63], the solution to the equation (7.17) is uniquely determined by imposing single-valued behaviour on $\hat{f}$.

First of all we note that any expression of the form $h(x)-h(\bar{x})$ obeys the homogeneous equation and antisymmetry under the exchange of $x$ and $\bar{x}$ and hence can be added to any solution of eq. (7.17). However, the conditions of single-valuedness,

$$
\left[\operatorname{disc}_{x}-\operatorname{disc}_{\bar{x}}\right] \hat{f}(x, \bar{x})=0, \quad\left[\operatorname{disc}_{1-x}-\operatorname{disc}_{1-\bar{x}}\right] \hat{f}(x, \bar{x})=0,
$$

and that 0 and 1 are the only singular points, fix this ambiguity.

Let us see how the ambiguity is fixed. Imagine that we have a single-valued solution and we try to add $h(x)-h(\bar{x})$ to it so that it remains a single-valued solution. Then the 
conditions (7.18) on the discontinuites tell us that $h$ can have no branch cuts at $x=0$ or $x=1$. Since these are the only places that the integral has any singularities, we conclude it has no branch cuts at all. Since the only singularities of the integral are logarithmic branch points, $h$ has no singularities at all and the only allowed possibility is that $h$ is constant, which drops out of the combination $h(x)-h(\bar{x})$. Thus there is indeed a unique single-valued solution to eq. (7.17). The argument we have just outlined is identical to the one used in ref. [63] to solve for the generalised ladders.

A very direct way of obtaining the symbol of the single-valued solution to eq. (7.17) is to make an ansatz of weight eight from the five letters

$$
\{x, 1-x, \bar{x}, 1-\bar{x}, 1-x \bar{x}\}
$$

and impose integrability and the initial entry condition. Then imposing that the differential equation is satisfied directly at symbol level leads to a unique answer.

\subsection{An integral solution}

Now let us look at the differential equation (7.17) in detail and construct the single-valued solution. It will be convenient to organise the right-hand side of the differential equation (7.17) according to symmetry under $x \leftrightarrow 1 / x$. We define

$$
\begin{aligned}
& E_{+}(x, \bar{x})=\frac{1}{2}\left[E(1-x, 1-\bar{x})+E\left(1-\frac{1}{x}, 1-\frac{1}{\bar{x}}\right)\right], \\
& E_{-}(x, \bar{x})=\frac{1}{2}\left[E(1-x, 1-\bar{x})-E\left(1-\frac{1}{x}, 1-\frac{1}{\bar{x}}\right)\right] .
\end{aligned}
$$

Then the differential equation reads

$$
(1-x \bar{x}) x \bar{x} \partial_{x} \partial_{\bar{x}} \hat{f}(x, \bar{x})=-(1-x \bar{x}) E_{-}(x, \bar{x})-(1+x \bar{x}) E_{+}(x, \bar{x}) .
$$

We may now split the equation (7.21) into two parts

$$
\begin{aligned}
x \bar{x} \partial_{x} \partial_{\bar{x}} f_{a}(x, \bar{x}) & =-E_{-}(x, \bar{x}) \\
(1-x \bar{x}) x \bar{x} \partial_{x} \partial_{\bar{x}} f_{b}(x, \bar{x}) & =-(1+x \bar{x}) E_{+}(x, \bar{x}) .
\end{aligned}
$$

Note that we may take both $f_{a}$ and $f_{b}$ to be antisymmetric under $x \leftrightarrow 1 / x$.

The equation (7.22) is of exactly the same form as the equations considered in ref. [63]. Following the prescription given in ref. [63], section 6.1, it is a simple matter to find a singlevalued solution to the equation (7.22) in terms of single-valued polylogs. We find

$$
\begin{aligned}
f_{a}(x, \bar{x})= & L_{3,4,0}-2 L_{4,3,0}+L_{5,2,0}+2 L_{3,2,2,0}-2 L_{4,1,2,0}-L_{4,2,0,0}-2 L_{4,2,1,0}+L_{5,0,0,0} \\
& +2 L_{5,1,0,0}+2 L_{5,1,1,0}+2 L_{4,1,1,0,0}-4 \zeta_{3}\left(\bar{L}_{5}-2 \bar{L}_{3,2}+2 \bar{L}_{4,0}+3 \bar{L}_{4,1}\right)
\end{aligned}
$$

We now treat the equation (7.23) for $f_{b}$. Let us split it into two parts so that $f_{b}(x, \bar{x})=$ $f_{1}(x, \bar{x})+f_{2}(x, \bar{x})$,

$$
(1-x \bar{x}) x \bar{x} \partial_{x} \partial_{\bar{x}} f_{1}(x, \bar{x})=-E_{+}(x, \bar{x})
$$




$$
(1-x \bar{x}) \partial_{x} \partial_{\bar{x}} f_{2}(x, \bar{x})=-E_{+}(x, \bar{x}) .
$$

We may write integral solutions

$$
f_{1}(x, \bar{x})=-\int_{1}^{x} \frac{d t}{t} \int_{1}^{\bar{x}} \frac{d \bar{t}}{\bar{t}} \frac{E_{+}(t, \bar{t})}{1-t \bar{t}}
$$

and

$$
f_{2}(x, \bar{x})=-f_{1}(1 / x, 1 / \bar{x})=-\int_{1}^{x} d t \int_{1}^{\bar{x}} d \bar{t} \frac{E_{+}(t, \bar{t})}{1-t \bar{t}}
$$

which obey the equations (7.25).

It follows that the full function $\hat{f}$ is given by

$$
\hat{f}(x, \bar{x})=f_{a}(x, \bar{x})+f_{1}(x, \bar{x})+f_{2}(x, \bar{x})+h(x)-h(\bar{x})
$$

for some holomorphic function $h$. We note that $\hat{f}(x, 1)-f_{a}(x, 1)=h(x)-h(1)$.

Now we examine the function $f_{2}$ in more detail. Writing $E_{+}(t, \bar{t})=\sum_{i} H_{w_{i}}(t) H_{w_{i}^{\prime}}(\bar{t})$ we find

$$
f_{2}(x, \bar{x})=\sum_{i} \int_{1}^{x} \frac{d t}{t} H_{w_{i}}(t) I_{w_{i}^{\prime}}(t, \bar{x})
$$

where, for a word $w$ made of the letters 0 and 1 ,

$$
I_{w}(x, \bar{x})=\int_{1}^{\bar{x}} \frac{d \bar{t}}{\bar{t}-1 / x} H_{w}(\bar{t})=(-1)^{d}\left(G\left(\frac{1}{x}, w ; \bar{x}\right)-G\left(\frac{1}{x}, w ; 1\right)\right) .
$$

We may now calculate the symbol of $f_{2}$. We note that

$$
d f_{2}(x, \bar{x})=d \log x \sum_{i} H_{w_{i}}(x) I_{w_{i}^{\prime}}(x, \bar{x})-(x \leftrightarrow \bar{x}) .
$$

The symbol of $I_{w}$ is obtained recursively using

$$
\mathcal{S}\left(I_{w}(x, \bar{x})\right)=\mathcal{S}\left(H_{w}(\bar{x})\right) \otimes \frac{1-x \bar{x}}{1-x a_{0}}-(-1)^{a_{0}} \mathcal{S}\left(I_{w^{\prime}}(x, \bar{x})\right) \otimes \frac{x}{1-x a_{0}},
$$

where $w=a_{0} w^{\prime}$. When $w$ is the empty word $I(x, \bar{x})$ is a logarithm,

$$
I(x, \bar{x})=\log \frac{1-x \bar{x}}{1-x} .
$$

Using the relations $(7.31),(7.32),(7.33)$ we obtain the symbol of $f_{2}(x, \bar{x})$. One finds that the result does not obey the initial entry condition (i.e. the first letters in the symbol are not only of the form $u=x \bar{x}$ or $v=(1-x)(1-\bar{x}))$. However, the inital entry condition can be uniquely restored by adding the symbols of single-variable functions in the form $\mathcal{S}\left(h_{2}(x)\right)-\mathcal{S}\left(h_{2}(\bar{x})\right)$. Inverting $x \leftrightarrow 1 / x$ we may similarly treat $f_{1}(x, \bar{x})=-f_{2}(1 / x, 1 / \bar{x})$. Combining everything we obtain the symbol

$$
\mathcal{S}(\hat{f}(x, \bar{x}))=\mathcal{S}\left(f_{a}(x, \bar{x})+f_{2}(x, \bar{x})+h_{2}(x)-h_{2}(\bar{x})-f_{2}(1 / x, 1 / \bar{x})-h_{2}(1 / x)+h_{2}(1 / \bar{x})\right) .
$$

The symbol obtained this way agrees with that obtained by imposing the differential equation on an ansatz as described around eq. (7.19). 
Given that single-valuedness uniquely determines the solution of the differential equation (7.17) one might suspect that we can use this property to give an explicit representation of the function $h_{2}(x)$. Indeed this is the case. The integral formula (7.29) can, in principle, have discontinuities around any of the five divisors obtained by setting a letter from the set (7.19) to zero.

Let us consider the discontinuity of $f_{2}(x, \bar{x})$ at $x=1 / \bar{x}$,

$$
\begin{aligned}
\operatorname{disc}_{x=1 / \bar{x}} f_{2}(x, \bar{x}) & =\int_{1 / \bar{x}}^{x} d t \operatorname{disc}_{t=1 / \bar{x}} \int_{1}^{\bar{x}} d \bar{t} \frac{E_{+}(t, \bar{t})}{1-t \bar{t}} \\
& =\int_{1 / \bar{x}}^{x} d t \operatorname{disc}_{\bar{x}=1 / t} \int_{1}^{\bar{x}} d \bar{t} \frac{E_{+}(t, \bar{t})}{1-t \bar{t}} \\
& =-\int_{1 / \bar{x}}^{x} \frac{d t}{t}(2 \pi i) E_{+}(t, 1 / t) .
\end{aligned}
$$

The above expression vanishes due to the symmetry of $E_{+}$under $x \leftrightarrow 1 / x$ and the antisymmetry under $x \leftrightarrow \bar{x}$. The absence of such discontinuities is the reason that we split the original equation into two pieces, one for $f_{a}$ and one for $f_{b}$.

Now let us consider the discontinuity around $x=1$. We find

$$
\begin{aligned}
\operatorname{disc}_{1-x} f_{2}(x, \bar{x}) & =-\int_{1}^{x} \frac{d t}{t} \operatorname{disc}_{1-t} \int_{1}^{\bar{x}} \frac{d \bar{t}}{\bar{t}-1 / t} E_{+}(t, \bar{t}) \\
& =-(2 \pi i) \int_{1}^{x} \frac{d t}{t} E_{+}(t, 1 / t)+\int_{1}^{x} d t \int_{1}^{\bar{x}} d \bar{t} \frac{\operatorname{disc}_{1-t} E_{+}(t, \bar{t})}{1-t \bar{t}} .
\end{aligned}
$$

The first term above again vanishes due to the symmetries of $E_{+}$. The second term will cancel against the corresponding term involving $\operatorname{disc}_{1-\bar{t}} E_{+}(t, \bar{t})$ in the integrand when we take the combination $\left[\operatorname{disc}_{1-x}-\operatorname{disc}_{1-\bar{x}}\right] f_{2}(x, \bar{x})$. The discontinuities at $x=1$ and $\bar{x}=1$ of $f_{2}$ therefore satisfy the single-valuedness conditions (7.18) since $E_{+}$does.

For the discontinuities at $x=0$ we find

$$
\operatorname{disc}_{x} f_{2}(x, \bar{x})=\int_{0}^{x} d t \operatorname{disc}_{t} \int_{1}^{\bar{x}} d \bar{t} \frac{E_{+}(t, \bar{t})}{1-t \bar{t}}=\int_{0}^{x} d t \int_{1}^{\bar{x}} \frac{d \bar{t}}{1-t \bar{t}} \operatorname{disc}_{t} E_{+}(t, \bar{t}) .
$$

Now writing the $\bar{t}$ integral above as $\int_{1}^{\bar{x}}=\int_{0}^{\bar{x}}-\int_{0}^{1}$ and using $\left[\operatorname{disc}_{t}-\operatorname{disc}_{\bar{t}}\right] E_{+}(t, \bar{t})=0$ we find

$$
\begin{aligned}
{\left[\operatorname{disc}_{x}-\operatorname{disc}_{\bar{x}}\right] f_{2}(x, \bar{x}) } & =\left[-\int_{0}^{x} d t \int_{0}^{1} \frac{d \bar{t}}{1-t \bar{t}} \operatorname{disc}_{t} E_{+}(t, \bar{t})\right]+(x \leftrightarrow \bar{x}) \\
& =\operatorname{disc}_{x}\left[-\int_{0}^{x} d t \int_{0}^{1} \frac{d \bar{t}}{1-t \bar{t}} E_{+}(t, \bar{t})\right]+(x \leftrightarrow \bar{x})
\end{aligned}
$$

Thus $f_{2}(x, \bar{x})$ is not single-valued by itself since the above combination of discontinuities (7.38) does not vanish. Note however that eq. (7.38) is of the form $k(x)+k(\bar{x})$, as necessary in order for it to be cancelled by adding a term of the form $h_{2}(x)-h_{2}(\bar{x})$ to $f_{2}(x, \bar{x})$. We now construct such a function $h_{2}(x)$.

Let

$$
h_{2}^{0}(x)=\int_{0}^{x} d t \int_{0}^{1} \frac{d \bar{t}}{1-t \bar{t}} E_{+}(t, \bar{t}) .
$$


Writing $E_{+}(t, \bar{t})=\sum_{i} H_{w_{i}}(t) H_{w_{i}^{\prime}}(\bar{t})$ we find

$$
h_{2}^{0}(x)=-\int_{0}^{x} \frac{d t}{t} \sum_{i} H\left(w_{i} ; t\right) \int_{0}^{1} \frac{d \bar{t}}{\bar{t}-1 / t} H\left(w_{i}^{\prime} ; \bar{t}\right) .
$$

We can write

$$
\int_{0}^{1} \frac{d \bar{t}}{\bar{t}-1 / t} H_{w_{i}^{\prime}}(\bar{t})=(-1)^{d} G\left(1 / t, w_{i}^{\prime}(0,1) ; 1\right),
$$

where we have made explicit that $w_{i}^{\prime}$ is a word in the letters 0 and 1 and $d$ is the number of 1 letters. One can always rewrite this in terms of HPLs at argument $t$. Indeed we can recursively apply the formula

$$
G\left(\frac{1}{t}, a_{2}, a_{3} \ldots, a_{n} ; 1\right)=\int_{0}^{t} \frac{d r}{r-1} G\left(\frac{a_{2}}{r}, a_{3}, \ldots, a_{n} ; 1\right)-\int_{0}^{t} \frac{d r}{r} G\left(\frac{1}{r}, a_{3}, \ldots, a_{n} ; 1\right) .
$$

to achieve this. Note that $a_{i} \in\{0,1\}$ in the above formula. We also need

$$
G\left(\frac{1}{t}, 0_{q} ; 1\right)=(-1)^{q+1} H_{q+1}(t) .
$$

Once this has been done, one can use standard HPL relations to calculate the products

$$
H_{w_{i}}(t) G\left(1 / t, w_{i}^{\prime} ; 1\right)
$$

and perform the remaining integral from 0 to $x$ w.r.t. $t$. We thus obtain a function $h_{2}^{0}$ whose discontinuity at $x=0$ is minus that of the $x$-dependent contribution to [ $\operatorname{disc}_{x}-$ $\left.\operatorname{disc}_{\bar{x}}\right] f_{2}(x, \bar{x})$.

In ref. [63] an explicit projection operator $\mathcal{F}$ was introduced which removes the discontinuity at $x=0$ of a linear combination of HPLs while preserving the discontinuity at $x=1$. The orthogonal projector $(1-\mathcal{F})$ removes the discontinuity at $x=1$ while preserving that at $x=0$. We define

$$
h_{2}(x)=(1-\mathcal{F}) h_{2}^{0}(x) .
$$

Explicitly we find

$$
\begin{aligned}
& h_{2}(x)=\frac{151}{16} \zeta_{6} H_{2}+\frac{15}{2} \zeta_{3}^{2} H_{2}-\frac{3}{2} \zeta_{2} \zeta_{3} H_{2,0}-\frac{15}{4} \zeta_{5} H_{2,0}-\zeta_{2} \zeta_{3} H_{2,1}+\frac{19}{4} \zeta_{4} H_{2,2}+2 \zeta_{3} H_{2,3} \\
& -\zeta_{2} H_{2,4}+\frac{21}{8} \zeta_{4} H_{2,0,0}+\frac{19}{4} \zeta_{4} H_{2,1,0}+\frac{17}{2} \zeta_{4} H_{2,1,1}+5 \zeta_{3} H_{2,1,2}-\zeta_{2} H_{2,1,3}-\frac{3}{2} \zeta_{3} H_{2,2,0} \\
& +\zeta_{3} H_{2,2,1}-\zeta_{2} H_{2,2,2}+\frac{1}{2} \zeta_{2} H_{2,3,0}-\zeta_{2} H_{2,3,1}-\frac{3}{2} \zeta_{3} H_{2,0,0,0}-3 \zeta_{3} H_{2,1,0,0}-3 \zeta_{3} H_{2,1,1,0} \\
& +4 \zeta_{3} H_{2,1,1,1}+\zeta_{2} H_{2,1,2,0}-2 \zeta_{2} H_{2,1,2,1}+\frac{1}{2} H_{2,1,4,0}+\zeta_{2} H_{2,2,1,0}+\frac{1}{2} H_{2,3,2,0}-\frac{1}{2} H_{2,4,0,0} \\
& \quad-H_{2,4,1,0}+\frac{1}{2} \zeta_{2} H_{2,1,0,0,0}+\zeta_{2} H_{2,1,1,0,0}+2 \zeta_{2} H_{2,1,1,1,0}+H_{2,1,1,3,0}+H_{2,1,2,2,0}-H_{2,1,3,0,0} \\
& +2 H_{2,1,1,1,2,0}-H_{2,1,3,1,0}+H_{2,2,1,2,0}-\frac{1}{2} H_{2,2,2,0,0}-H_{2,2,2,1,0}+\frac{1}{2} H_{2,3,0,0,0}-H_{2,3,1,1,0}
\end{aligned}
$$




$$
\begin{aligned}
& -H_{2,1,1,2,0,0}+\frac{1}{2} H_{2,1,2,0,0,0}-H_{2,1,2,1,0,0}-2 H_{2,1,2,1,1,0}+H_{2,2,1,0,0,0}+H_{2,2,1,1,0,0} \\
& +H_{2,1,1,1,0,0,0}
\end{aligned}
$$

Here the $H$ functions are all implicitly evaluated at argument $x$.

The contribution from $f_{1}(x, \bar{x})=-f_{2}(1 / x, 1 / \bar{x})$ is made single-valued by inversion on $x$. So we define

$$
h(x)=h_{2}(x)-h_{2}(1 / x) .
$$

Finally we deduce that the combination

$$
\hat{f}(x, \bar{x})=f_{a}(x, \bar{x})+f_{1}(x, \bar{x})+f_{2}(x, \bar{x})+h(x)-h(\bar{x})
$$

is single-valued and obeys the differential equation (7.17) and hence describes the fourloop integral $I^{(4)}$ defined in equation (7.1). The equations (7.29), (7.46), (7.47) and (7.48) therefore explicitly define the function $\hat{f}$.

\subsection{Expression in terms of multiple polylogarithms}

Now let us rewrite the integral form (7.29), (7.30) for $f_{2}(x, \bar{x})$ in terms of multiple polylogarithms. We use the following generalisation of relation (7.42),

$$
\begin{aligned}
G\left(\frac{1}{y}, a_{2}, \ldots, a_{n} ; z\right)=( & \left.\left(\frac{1}{z} ; y\right)-G\left(\frac{1}{a_{2}} ; y\right)\right) G\left(a_{2}, \ldots, a_{n} ; z\right) \\
& +\int_{0}^{y}\left(\frac{d t}{t-\frac{1}{a_{2}}}-\frac{d t}{t}\right) G\left(\frac{1}{t}, a_{3}, \ldots, a_{n} ; z\right)
\end{aligned}
$$

to recursively rewrite the $G\left(\frac{1}{t}, \ldots\right)$ appearing in the $I_{w_{i}^{\prime}}(t, \bar{x})$ in eq. (7.29) so that the $t$ appears as the final argument. Note that in eq. (7.49), the two terms involving an explicit appearance of $1 / a_{2}$ vanish in the case $a_{2}=0$. The recursion begins with

$$
G\left(\frac{1}{y} ; z\right)=\log (1-y z)=G\left(\frac{1}{z} ; y\right) .
$$

The recursion allows us to write the products $H_{w_{i}}(t) I_{w_{i}^{\prime}}(t, \bar{x})$ as a sum of multiple polylogarithms of the form $G(w ; t)$ where the weight vectors depend on $\bar{x}$. Then we can perform the final integration $d t / t$ to obtain an expression for $f_{2}$ in terms of multiple polylogs.

We may relate $f_{1}(x, \bar{x})$ directly to $f_{2}(x, \bar{x})$ since

$$
\begin{aligned}
f_{1}(x, \bar{x}) & =-\int_{1}^{x} \frac{d t}{t} \int_{1}^{\bar{x}} \frac{d \bar{t}}{\bar{t}} \frac{E_{+}(t, \bar{t})}{1-t \bar{t}}=\int_{1}^{x} \frac{d t}{t^{2}} \int_{1}^{\bar{x}} \frac{d \bar{t}}{\bar{t}(\bar{t}-1 / t)} E_{+}(t, \bar{t}) \\
& =\int_{1}^{x} \frac{d t}{t}\left[\int_{1}^{\bar{x}} \frac{d \bar{t}}{\bar{t}-1 / t} E_{+}(t, \bar{t})-\int_{1}^{\bar{x}} \frac{d \bar{t}}{\bar{t}} E_{+}(t, \bar{t})\right] \\
& =f_{2}(x, \bar{x})-\sum_{i}\left[H_{0 w_{i}}(x)-H_{0 w_{i}}(1)\right]\left[H_{0 w_{i}^{\prime}}(\bar{x})-H_{0 w_{i}^{\prime}}(1)\right] .
\end{aligned}
$$

For a practical scheme we express $E_{+}$as a sum over $H_{w_{i}}(t) H_{w_{i}^{\prime}}(\bar{t})$ and do the $\bar{t}$ integration. In any single term of the integrand of $f_{2}$, the recursion (7.49) will lead to multiple polylogarithms of the type $G(\ldots, 1 / \bar{x} ; t)$. Next, we take the shuffle product with the second polylogarithm and integrate over $t$. 
In this raw form our result is not manifestly antisymmetric under $x \leftrightarrow \bar{x}$. Remarkably, in the sum over all terms only $G(0,1 / \bar{x}, \ldots ; x)$ remain. Upon rewriting

$$
G\left(0, \frac{1}{\bar{x}}, a_{1}, \ldots, a_{n} ; x\right)=G(0 ; x) G\left(\frac{1}{\bar{x}}, a_{1}, \ldots, a_{n} ; x\right)-\int_{0}^{x} \frac{d t}{t-\frac{1}{\bar{x}}} G(0 ; t) G\left(a_{1}, \ldots, a_{n} ; t\right)
$$

we can use (7.49) to swap $G(1 / \bar{x}, \ldots ; x)$ for (a sum over) $G(\ldots, 1 / x ; \bar{x})$. Replacing the original two-variable polylogarithms by $1 / 2$ themselves and $1 / 2$ the $x, \bar{x}$ swapped version, we can obtain a manifestly antisymmetric form. The shuffle algebra is needed to remove zeroes from the rightmost position of the weight vectors and to bring the letters $1 / x, 1 / \bar{x}$ to the left of all entries 1 . Finally we rescale to argument 1.

In analogy to the notation introduced for the Hard integral let us write

$$
G_{\hat{3}, 2,1}=G\left(0,0, \frac{1}{x \bar{x}}, 0, \frac{1}{x}, \frac{1}{x} ; 1\right)
$$

etc. Collecting terms, we find

$$
\begin{aligned}
& I_{14 ; 23}^{(4)}(x, \bar{x})= \\
& -L_{2,2,4}+2 L_{2,3,3}-L_{2,4,2}-2 L_{2,1,1,4}+2 L_{2,1,2,3}-2 L_{2,1,3,2}+2 L_{2,1,4,1}+2 L_{2,2,1,3}-2 L_{2,2,2,2} \\
& -2 L_{2,2,3,1}+2 L_{2,3,1,2}+2 L_{2,3,2,0}+2 L_{2,3,2,1}-2 L_{2,4,1,0}-2 L_{2,4,1,1}-2 L_{3,1,3,0}+2 L_{3,3,1,0} \\
& -4 L_{2,1,1,2,2}+4 L_{2,1,2,2,1}+4 L_{2,2,1,1,2}-4 L_{2,2,2,1,0}-4 L_{2,2,2,1,1}-4 L_{3,1,1,2,0}+2 L_{3,2,1,0,0} \\
& +4 L_{3,2,1,1,0}+L_{0}\left(-H_{1,2,4}^{-}+2 H_{1,3,3}^{-}-H_{1,4,2}^{-}-2 H_{1,1,1,4}^{-}+2 H_{1,1,2,3}^{-}-2 H_{1,1,3,2}^{-}+2 H_{1,1,4,1}^{-}\right. \\
& +2 H_{1,2,1,3}^{-}-2 H_{1,2,2,2}^{-}-2 H_{1,2,3,1}^{-}+2 H_{1,3,1,2}^{-}+2 H_{1,3,2,1}^{-}-2 H_{1,4,1,1}^{-}-4 H_{1,1,1,2,2}^{-}+4 H_{1,1,2,2,1}^{-} \\
& \left.+4 H_{1,2,1,1,2}^{-}-4 H_{1,2,2,1,1}^{-}\right)+4 H_{1,2,5}^{-}-4 H_{1,3,4}^{-}-4 H_{1,4,3}^{-}+4 H_{1,5,2}^{-}+8 H_{1,1,1,5}^{-}-4 H_{1,1,2,4}^{-} \\
& +4 H_{1,1,4,2}^{-}-8 H_{1,1,5,1}^{-}-4 H_{1,2,1,4}^{-}+8 H_{1,2,3,2}^{-}+4 H_{1,2,4,1}^{-}-8 H_{1,3,1,3}^{-}-4 H_{1,4,1,2}^{-}-4 H_{1,4,2,1}^{-} \\
& +8 H_{1,5,1,1}^{-}+8 H_{1,1,1,2,3}^{-}+8 H_{1,1,1,3,2}^{-}-8 H_{1,1,2,3,1}^{-}-8 H_{1,1,3,2,1}^{-}-8 H_{1,2,1,1,3}^{-}+8 H_{1,2,3,1,1}^{-} \\
& -8 H_{1,3,1,1,2}^{-}+8 H_{1,3,2,1,1}^{-}+\zeta_{3}\left(8 \bar{L}_{2,3}-12 \bar{L}_{3,2}-12 \bar{L}_{2,1,2}+12 \bar{L}_{2,2,1}-12 \bar{L}_{3,1,0}-16 \bar{L}_{3,1,1}\right. \\
& \left.-12 L_{0} H_{1,1,2}^{-}+12 L_{0} H_{1,2,1}^{-}+16 H_{1,1,3}^{-}-16 H_{1,3,1}^{-}-16 H_{1,1,1,2}^{-}+16 H_{1,2,1,1}^{-}\right)+2 \bar{L}_{3} \zeta_{5} \\
& +\zeta_{3}^{2}\left(-24 L_{2}-72 H_{2}^{-}-48 H_{1,1}^{-}\right)+ \\
& G_{\hat{2}}^{+}\left(-L_{4,2}-L_{4,0,0}-2 L_{4,1,0}-2 L_{4,1,1}-4 \bar{L}_{2,1} \zeta_{3}+4 L_{0} \zeta_{3} H_{2}^{-}+4 L_{1} \zeta_{3} H_{2}^{-}\right. \\
& +3 \bar{L}_{2} H_{4}^{-}+2 \bar{L}_{1,1} H_{4}^{-}-4 L_{1} H_{5}^{-}+4 \zeta_{3} H_{1,2}^{-}-2 \bar{L}_{0,0} H_{1,3}^{-}+5 L_{0} H_{1,4}^{-}-2 L_{1} H_{1,4}^{-}-12 H_{1,5}^{-} \\
& +12 \zeta_{3} H_{2,1}^{-}-\bar{L}_{0,0} H_{2,2}^{-}+2 L_{0} H_{2,3}^{-}-2 L_{1} H_{2,3}^{-}-6 H_{2,4}^{-}+L_{0} H_{3,2}^{-}-2 L_{1} H_{3,2}^{-}-4 L_{0} H_{4,1}^{-} \\
& -2 L_{1} H_{4,1}^{-}+2 H_{4,2}^{-}+16 H_{5,1}^{-}+16 \zeta_{3} H_{1,1,1}^{-}-2 \bar{L}_{0,0} H_{1,1,2}^{-}+6 L_{0} H_{1,1,3}^{-}-12 H_{1,1,4}^{-}-2 \bar{L}_{0,0} H_{1,2,1}^{-} \\
& +6 L_{0} H_{1,2,2}^{-}-8 H_{1,2,3}^{-}+2 L_{0} H_{1,3,1}^{-}-8 H_{1,3,2}^{-}+4 H_{1,4,1}^{-}+2 \bar{L}_{0,0} H_{2,1,1}^{-}+2 L_{0} H_{2,1,2}^{-}-4 H_{2,1,3}^{-} \\
& -2 L_{0} H_{2,2,1}^{-}-4 H_{2,2,2}^{-}+4 H_{2,3,1}^{-}-6 L_{0} H_{3,1,1}^{-}+4 H_{3,1,2}^{-}+12 H_{3,2,1}^{-}+12 H_{4,1,1}^{-} \\
& \left.+4 L_{0} H_{1,1,1,2}^{-}-8 H_{1,1,1,3}^{-}-8 H_{1,1,2,2}^{-}-4 L_{0} H_{1,2,1,1}^{-}+8 H_{1,2,2,1}^{-}+8 H_{1,3,1,1}^{-}-L_{2} H_{4}^{+}\right)+ \\
& G_{\hat{3}}^{+}\left(2 \bar{L}_{3,2}+4 \bar{L}_{3,1,0}+4 \bar{L}_{3,1,1}-8 \zeta_{3} H_{2}^{-}-6 \bar{L}_{2} H_{3}^{-}-2 \bar{L}_{0,0} H_{3}^{-}-4 \bar{L}_{1,1} H_{3}^{-}+8 L_{0} H_{4}^{-}\right. \\
& +6 L_{1} H_{4}^{-}-20 H_{5}^{-}-8 \zeta_{3} H_{1,1}^{-}+8 L_{0} H_{1,3}^{-}+4 L_{1} H_{1,3}^{-}-20 H_{1,4}^{-}+6 L_{0} H_{2,2}^{-}+4 L_{1} H_{2,2}^{-}
\end{aligned}
$$




$$
\begin{aligned}
& -16 H_{2,3}^{-}+4 L_{0} H_{3,1}^{-}+4 L_{1} H_{3,1}^{-}-16 H_{3,2}^{-}-8 H_{4,1}^{-}+4 L_{0} H_{1,1,2}^{-}-16 H_{1,1,3}^{-}+4 L_{0} H_{1,2,1}^{-} \\
& \left.-16 H_{1,2,2}^{-}-8 H_{1,3,1}^{-}-4 L_{0} H_{2,1,1}^{-}-8 H_{2,1,2}^{-}+8 H_{3,1,1}^{-}-8 H_{1,1,1,2}^{-}+8 H_{1,2,1,1}^{-}+2 L_{2} H_{3}^{+}\right)+ \\
& G_{\hat{2}, 1}^{+}\left(2 \bar{L}_{3,2}-\bar{L}_{4,0}-2 \bar{L}_{4,1}+2 \bar{L}_{3,1,0}-12 L_{2} \zeta_{3}+16 \zeta_{3} H_{2}^{-}-4 \bar{L}_{2} H_{3}^{-}\right. \\
& -2 \bar{L}_{0,0} H_{3}^{-}+8 L_{0} H_{4}^{-}+8 L_{1} H_{4}^{-}-20 H_{5}^{-}+16 \zeta_{3} H_{1,1}^{-}+4 L_{0} H_{1,3}^{-}-16 H_{1,4}^{-}+4 L_{0} H_{2,2}^{-} \\
& \left.-12 H_{2,3}^{-}-12 H_{3,2}^{-}+4 L_{0} H_{1,1,2}^{-}-8 H_{1,1,3}^{-}-8 H_{1,2,2}^{-}-4 L_{0} H_{2,1,1}^{-}+8 H_{2,2,1}^{-}+8 H_{3,1,1}^{-}\right)+ \\
& G_{\hat{4}}^{+}\left(3 L_{0} H_{3}^{-}-12 H_{4}^{-}+3 L_{0} H_{1,2}^{-}-12 H_{1,3}^{-}+6 L_{0} H_{2,1}^{-}-12 H_{2,2}^{-}-12 H_{3,1}^{-}+6 L_{0} H_{1,1,1}^{-}\right. \\
& \left.-12 H_{1,1,2}^{-}-12 H_{1,2,1}^{-}\right)+G_{\hat{3}, 1}^{+}\left(2 L_{3,0}+4 L_{3,1}-8 \zeta_{3} H_{1}^{-}+2 L_{0} H_{3}^{-}-4 L_{1} H_{3}^{-}-8 H_{4}^{-}\right. \\
& \left.+4 L_{0} H_{1,2}^{-}-8 H_{1,3}^{-}+4 L_{0} H_{2,1}^{-}-8 H_{2,2}^{-}-8 H_{1,1,2}^{-}+8 H_{2,1,1}^{-}\right)+G_{\hat{2}, 2}^{+}\left(L_{4}+L_{3,0}+4 L_{3,1}\right. \\
& \left.-16 \zeta_{3} H_{1}^{-}-4 L_{1} H_{3}^{-}+4 H_{1,3}^{-}+4 H_{2,2}^{-}+8 H_{3,1}^{-}-4 L_{0} H_{1,1,1}^{-}+8 H_{1,2,1}^{-}+8 H_{2,1,1}^{-}\right)+ \\
& G_{\hat{2}, 1,1}^{+}\left(-2 L_{4}+2 L_{3,0}+16 \zeta_{3} H_{1}^{-}+4 L_{0} H_{1,2}^{-}-8 H_{1,3}^{-}-8 H_{2,2}^{-}\right)+ \\
& G_{\hat{\tilde{5}}}^{+}\left(-4 H_{3}^{-}-4 H_{1,2}^{-}-8 H_{2,1}^{-}-8 H_{1,1,1}^{-}\right)+G_{\hat{4}, 1}^{+}\left(-6 \bar{L}_{2,1}+3 L_{0} H_{2}^{-}+6 L_{1} H_{2}^{-}-12 H_{3}^{-}\right. \\
& \left.-12 H_{1,2}^{-}-12 H_{2,1}^{-}\right)+G_{\hat{3}, 2}^{+}\left(-2 \bar{L}_{3}-8 \bar{L}_{2,1}+2 L_{0} H_{2}^{-}+8 L_{1} H_{2}^{-}-4 H_{3}^{-}-8 H_{1,2}^{-}\right. \\
& \left.-8 H_{2,1}^{-}+8 H_{1,1,1}^{-}\right)+G_{\hat{3}, 1,1}^{+}\left(4 \bar{L}_{3}+4 L_{0} H_{2}^{-}-16 H_{3}^{-}-8 H_{1,2}^{-}\right)+G_{\hat{2}, 3}^{+}\left(-4 \bar{L}_{3}-8 \bar{L}_{2,1}\right. \\
& \left.+2 L_{0} H_{2}^{-}+8 L_{1} H_{2}^{-}-4 H_{1,2}^{-}-8 H_{2,1}^{-}+8 H_{1,1,1}^{-}\right)+G_{\hat{2}, 2,1}^{+}\left(4 \bar{L}_{3}-8 H_{3}^{-}-8 H_{1,2}^{-}\right)+ \\
& G_{\hat{2}, 1,2}^{+}\left(4 \bar{L}_{3}+4 \bar{L}_{2,1}-4 L_{1} H_{2}^{-}-4 H_{3}^{-}+8 H_{2,1}^{-}\right)+G_{\hat{2}, 1,1,1}^{+}\left(4 L_{0} H_{2}^{-}-8 H_{3}^{-}\right)+ \\
& \left(10 G_{\hat{2}, 4}^{+}+8 G_{\hat{3}, 3}^{+}+3 G_{\hat{4}, 2}^{+}-8 G_{\hat{2}, 1,3}^{+}-8 G_{\hat{2}, 2,2}^{+}-4 G_{\hat{2}, 3,1}^{+}-8 G_{\hat{3}, 1,2}^{+}-8 G_{\hat{3}, 2,1}^{+}-6 G_{\hat{4}, 1,1}^{+}\right. \\
& \left.+4 G_{\hat{2}, 1,1,2}^{+}-4 G_{\hat{2}, 2,1,1}^{+}\right) L_{2}+\left(-16 G_{\hat{2}, 4}^{+}-12 G_{\hat{3}, 3}^{+}-6 G_{\hat{4}, 2}^{+}-4 G_{\hat{5}, 1}^{+}+12 G_{\hat{2}, 1,3}^{+}\right. \\
& \left.+12 G_{\hat{2}, 2,2}^{+}+8 G_{\hat{2}, 3,1}^{+}+8 G_{\hat{\mathrm{z}}, 1,2}^{+}+8 G_{\hat{3}, 2,1}^{+}-8 G_{\hat{2}, 1,1,2}^{+}-8 G_{\hat{2}, 1,2,1}^{+}-8 G_{\hat{\mathrm{3}}, 1,1,1}^{+}\right) H_{2}^{-}+ \\
& \left(-16 G_{\hat{2}, 4}^{+}-16 G_{\hat{3}, 3}^{+}-12 G_{\hat{4}, 2}^{+}-8 G_{\hat{5}, 1}^{+}+8 G_{\hat{2}, 1,3}^{+}+8 G_{\hat{2}, 2,2}^{+}+8 G_{\hat{3}, 1,2}^{+}\right) H_{1,1}^{-}+ \\
& \left(20 G_{\hat{2}, 5}^{+}+20 G_{\hat{3}, 4}^{+}+12 G_{\hat{4}, 3}^{+}+4 G_{\hat{5}, 2}^{+}-16 G_{\hat{2}, 1,4}^{+}-12 G_{\hat{2}, 2,3}^{+}-12 G_{\hat{2}, 3,2}^{+}-16 G_{\hat{3}, 1,3}^{+}\right. \\
& -16 G_{\hat{3}, 2,2}^{+}-8 G_{\hat{3}, 3,1}^{+}-12 G_{\hat{4}, 1,2}^{+}-12 G_{\hat{4}, 2,1}^{+}-8 G_{\hat{5}, 1,1}^{+}+8 G_{\hat{2}, 1,1,3}^{+}+8 G_{\hat{2}, 1,2,2}^{+}-8 G_{\hat{2}, 2,2,1}^{+} \\
& \left.-8 G_{\hat{2}, 3,1,1}^{+}+8 G_{\hat{3}, 1,1,2}^{+}-8 G_{\hat{3}, 2,1,1}^{+}\right) H_{1}^{-}+ \\
& G_{\hat{2}, 1}^{-}\left(2 L_{3,2}-L_{4,0}-2 L_{4,1}+L_{3,0,0}+2 L_{3,1,0}-12 \bar{L}_{2} \zeta_{3}+8 \zeta_{3} H_{2}^{+}-4 \bar{L}_{2} H_{3}^{+}\right. \\
& +8 L_{1} H_{4}^{+}-16 \zeta_{3} H_{1,1}^{+}-4 H_{2,3}^{+}+4 L_{0} H_{3,1}^{+}-4 H_{3,2}^{+}-16 H_{4,1}^{+}-4 L_{0} H_{1,1,2}^{+}+8 H_{1,1,3}^{+} \\
& \left.+8 H_{1,2,2}^{+}+4 L_{0} H_{2,1,1}^{+}-8 H_{2,2,1}^{+}-8 H_{3,1,1}^{+}\right)+ \\
& G_{\hat{3}, 1}^{-}\left(2 \bar{L}_{3,0}+4 \bar{L}_{3,1}+4 L_{1} \zeta_{3}-6 L_{0} H_{3}^{+}-4 L_{1} H_{3}^{+}+20 H_{4}^{+}-4 L_{0} H_{1,2}^{+}+16 H_{1,3}^{+}\right. \\
& \left.-4 L_{0} H_{2,1}^{+}+16 H_{2,2}^{+}+8 H_{3,1}^{+}+8 H_{1,1,2}^{+}-8 H_{2,1,1}^{+}\right)+G_{\hat{2}, 2}^{-}\left(\bar{L}_{4}+\bar{L}_{3,0}+4 \bar{L}_{3,1}+8 L_{1} \zeta_{3}\right. \\
& \left.-2 L_{0} H_{3}^{+}-4 L_{1} H_{3}^{+}+4 H_{4}^{+}+4 H_{1,3}^{+}+4 H_{2,2}^{+}+4 L_{0} H_{1,1,1}^{+}-8 H_{1,2,1}^{+}-8 H_{2,1,1}^{+}\right)+ \\
& G_{\hat{2}, 1,1}^{-}\left(-2 \bar{L}_{4}+2 \bar{L}_{3,0}-8 L_{1} \zeta_{3}-4 L_{0} H_{3}^{+}+16 H_{4}^{+}-4 L_{0} H_{1,2}^{+}+8 H_{1,3}^{+}+8 H_{2,2}^{+}\right)+ \\
& G_{\hat{4}, 1}^{-}\left(-3 L_{2,0}-6 L_{2,1}+3 L_{0} H_{2}^{+}+6 L_{1} H_{2}^{+}\right)+G_{\hat{3}, 2}^{-}\left(-2 L_{3}-2 L_{2,0}-8 L_{2,1}+\right.
\end{aligned}
$$




$$
\begin{aligned}
& \left.2 L_{0} H_{2}^{+}+8 L_{1} H_{2}^{+}-8 H_{1,2}^{+}-8 H_{2,1}^{+}-8 H_{1,1,1}^{+}\right)+G_{\hat{3}, 1,1}^{-}\left(4 L_{3}-2 L_{2,0}+8 H_{1,2}^{+}\right)+ \\
& G_{\hat{2}, 3}^{-}\left(-4 L_{3}-2 L_{2,0}-8 L_{2,1}-L_{0,0,0}-8 \zeta_{3}+2 L_{0} H_{2}^{+}+8 L_{1} H_{2}^{+}-12 H_{1,2}^{+}\right. \\
& \left.-8 H_{2,1}^{+}-8 H_{1,1,1}^{+}\right)+G_{\hat{2}, 2,1}^{-}\left(4 L_{3}+2 L_{0,0,0}+16 \zeta_{3}+8 H_{1,2}^{+}\right)+G_{\hat{2}, 1,2}^{-}\left(4 L_{3}+L_{2,0}\right. \\
& \left.+4 L_{2,1}+L_{0,0,0}+8 \zeta_{3}-2 L_{0} H_{2}^{+}-4 L_{1} H_{2}^{+}+8 H_{1,2}^{+}\right)+G_{\hat{2}, 1,1,1}^{-}\left(-2 L_{2,0}-2 L_{0,0,0}-16 \zeta_{3}\right)+ \\
& G_{\hat{\mathrm{5}}, 1}^{-}\left(4 H_{2}^{+}+8 H_{1,1}^{+}\right)+G_{\hat{4}, 2}^{-}\left(3 \bar{L}_{2}+12 H_{1,1}^{+}\right)+G_{\hat{4}, 1,1}^{-}\left(-6 \bar{L}_{2}+12 H_{2}^{+}\right)+G_{\hat{3}, 3}^{-}\left(8 \bar{L}_{2}+2 \bar{L}_{0,0}\right. \\
& \left.-4 H_{2}^{+}+16 H_{1,1}^{+}\right)+G_{\hat{3}, 2,1}^{-}\left(-8 \bar{L}_{2}-4 \bar{L}_{0,0}+8 H_{2}^{+}\right)+G_{\hat{3}, 1,2}^{-}\left(-8 \bar{L}_{2}-2 \bar{L}_{0,0}+8 H_{2}^{+}\right. \\
& \left.-8 H_{1,1}^{+}\right)+G_{\hat{3}, 1,1,1}^{-}\left(4 \bar{L}_{0,0}+8 H_{2}^{+}\right)+G_{\hat{2}, 4}^{-}\left(10 \bar{L}_{2}+4 \bar{L}_{0,0}-4 H_{2}^{+}+16 H_{1,1}^{+}\right)+ \\
& G_{\hat{2}, 3,1}^{-}\left(-4 \bar{L}_{2}-4 \bar{L}_{0,0}\right)+G_{\hat{2}, 2,2}^{-}\left(-8 \bar{L}_{2}-4 \bar{L}_{0,0}+4 H_{2}^{+}-8 H_{1,1}^{+}\right)+G_{\hat{2}, 2,1,1}^{-}\left(-4 \bar{L}_{2}+8 H_{2}^{+}\right)+ \\
& G_{\hat{2}, 1,3}^{-}\left(-8 \bar{L}_{2}-4 \bar{L}_{0,0}+4 H_{2}^{+}-8 H_{1,1}^{+}\right)+G_{\hat{2}, 1,2,1}^{-}\left(4 \bar{L}_{0,0}+8 H_{2}^{+}\right)+G_{\hat{2}, 1,1,2}^{-}\left(4 \bar{L}_{2}+4 \bar{L}_{0,0}\right)+ \\
& \left(-10 G_{\hat{2}, 5}^{-}-8 G_{\hat{3}, 4}^{-}-3 G_{\hat{4}, 3}^{-}+10 G_{\hat{2}, 1,4}^{-}+8 G_{\hat{2}, 2,3}^{-}+8 G_{\hat{2}, 3,2}^{-}+4 G_{\hat{2}, 4,1}^{-}+8 G_{\hat{3}, 1,3}^{-}\right. \\
& +8 G_{\hat{3}, 2,2}^{-}+8 G_{\hat{3}, 3,1}^{-}+3 G_{\hat{4}, 1,2}^{-}+6 G_{\hat{4}, 2,1}^{-}-8 G_{\hat{2}, 1,1,3}^{-}-8 G_{\hat{2}, 1,2,2}^{-}-4 G_{\hat{2}, 1,3,1}^{-}-4 G_{\hat{2}, 2,1,2}^{-} \\
& \left.+4 G_{\hat{2}, 3,1,1}^{-}-8 G_{\hat{3}, 1,1,2}^{-}-8 G_{\hat{3}, 1,2,1}^{-}-6 G_{\hat{4}, 1,1,1}^{-}+4 G_{\hat{2}, 1,1,1,2}^{-}-4 G_{\hat{2}, 1,2,1,1}^{-}\right) L_{0}+ \\
& \left(-10 G_{\hat{\hat{2}, 5}}^{-}-10 G_{\hat{\mathrm{3}}, 4}^{-}-6 G_{\hat{\mathrm{u}, 3}}^{-}-2 G_{\hat{\mathrm{5}, 2}}^{-}+8 G_{\hat{\mathrm{2}, 1,4}}^{-}+6 G_{\hat{2}, 2,3}^{-}+6 G_{\hat{\hat{2}, 3,2}}^{-}+8 G_{\hat{\mathrm{j}, 1,3}}^{-}\right. \\
& +8 G_{\hat{\mathrm{j}, 2,2}}^{-}+4 G_{\hat{3}, 3,1}^{-}+6 G_{\hat{4}, 1,2}^{-}+6 G_{\hat{4}, 2,1}^{-}+4 G_{\hat{\mathrm{5}, 1,1}}^{-}-4 G_{\hat{2}, 1,1,3}^{-}-4 G_{\hat{2}, 1,2,2}^{-}+4 G_{\hat{2}, 2,2,1}^{-} \\
& \left.+4 G_{\hat{2}, 3,1,1}^{-}-4 G_{\hat{3}, 1,1,2}^{-}+4 G_{\hat{3}, 2,1,1}^{-}\right) L_{1}+ \\
& 20 G_{\hat{2}, 6}^{-}+20 G_{\hat{3}, 5}^{-}+12 G_{\hat{4}, 4}^{-}+4 G_{\hat{5}, 3}^{-}-20 G_{\hat{2}, 1,5}^{-}-16 G_{\hat{2}, 2,4}^{-}-12 G_{\hat{2}, 3,3}^{-}-12 G_{\hat{2}, 4,2}^{-} \\
& -20 G_{\hat{3}, 1,4}^{-}-16 G_{\hat{3}, 2,3}^{-}-16 G_{\hat{3}, 3,2}^{-}-8 G_{\hat{3}, 4,1}^{-}-12 G_{\hat{4}, 1,3}^{-}-12 G_{\hat{4}, 2,2}^{-}-12 G_{\hat{4}, 3,1}^{-}-4 G_{\hat{\hat{j}, 1,2}}^{-} \\
& -8 G_{\hat{5}, 2,1}^{-}+16 G_{\hat{2}, 1,1,4}^{-}+12 G_{\hat{2}, 1,2,3}^{-}+12 G_{\hat{2}, 1,3,2}^{-}+8 G_{\hat{2}, 2,1,3}^{-}+8 G_{\hat{2}, 2,2,2}^{-}-8 G_{\hat{2}, 3,2,1}^{-}-8 G_{\hat{2}, 4,1,1}^{-} \\
& +16 G_{\hat{3}, 1,1,3}^{-}+16 G_{\hat{3}, 1,2,2}^{-}+8 G_{\hat{3}, 1,3,1}^{-}+8 G_{\hat{3}, 2,1,2}^{-}-8 G_{\hat{3}, 3,1,1}^{-}+12 G_{\hat{4}, 1,1,2}^{-}+12 G_{\hat{4}, 1,2,1}^{-}+8 G_{\hat{\hat{j}, 1,1,1}}^{-} \\
& -8 G_{\hat{2}, 1,1,1,3}^{-}-8 G_{\hat{2}, 1,1,2,2}^{-}+8 G_{\hat{2}, 1,2,2,1}^{-}+8 G_{\hat{2}, 1,3,1,1}^{-}-8 G_{\hat{3}, 1,1,1,2}^{-}+8 G_{\hat{3}, 1,2,1,1}^{-}
\end{aligned}
$$

\subsection{Numerical consistency tests for $I^{(4)}$}

In order to check the correctness of the result from the previous section, we evaluated $I^{(4)}$ numerically and compared it to a direct numerical evaluation of the coordinate space integral using FIESTA. In detail, we evaluate the conformally-invariant function $f(u, v)=$ $x_{13}^{2} x_{24}^{2} I^{(4)}\left(x_{1}, x_{2}, x_{3}, x_{4}\right)$ by first applying a conformal transformation to send $x_{4}$ to infinity, the integral takes the simplified form,

$$
\lim _{x_{4} \rightarrow \infty} x_{13}^{2} x_{24}^{2} I_{14 ; 23}^{(4)}=\frac{1}{\pi^{8}} \int \frac{d^{4} x_{5} d^{4} x_{6} d^{4} x_{7} d^{4} x_{8} x_{13}^{2}}{x_{15}^{2} x_{18}^{2} x_{25}^{2} x_{26}^{2} x_{37}^{2} x_{38}^{2} x_{56}^{2} x_{67}^{2} x_{78}^{2}},
$$

and then using the remaining freedom to fix $x_{13}^{2}=1$ so that $u=x_{12}^{2}$ and $v=x_{23}^{2}$. In comparison with the two 3-loop integrals, the extra loop in this case yields a moderately more cumbersome numerical evaluation. As such, we modify the setup for the 3-loop examples slightly and only perform $5 \times 10^{5}$ integral evaluations. We nevertheless obtain about 5 dig- 


\begin{tabular}{|ccccc|}
\hline$u$ & $v$ & Analytic & FIESTA & $\delta$ \\
\hline 0.1 & 0.2 & 156.733 & 156.733 & $4.9 \mathrm{e}-7$ \\
0.2 & 0.3 & 116.962 & 116.962 & $5.9 \mathrm{e}-8$ \\
0.3 & 0.1 & 110.366 & 110.366 & $2.8 \mathrm{e}-7$ \\
0.4 & 0.5 & 84.2632 & 84.2632 & $1.4 \mathrm{e}-7$ \\
0.5 & 0.6 & 75.2575 & 75.2575 & $1.4 \mathrm{e}-7$ \\
0.6 & 0.2 & 78.3720 & 78.3720 & $3.7 \mathrm{e}-8$ \\
0.7 & 0.3 & 70.7417 & 70.7417 & $6.8 \mathrm{e}-8$ \\
0.8 & 0.9 & 58.6362 & 58.6363 & $1.4 \mathrm{e}-7$ \\
0.9 & 0.5 & 60.1295 & 60.1295 & $1.1 \mathrm{e}-7$ \\
\hline
\end{tabular}

Table 4. Numerical comparison of the analytic result for $x_{13}^{2} x_{24}^{2} I^{(4)}\left(x_{1}, x_{2}, x_{3}, x_{4}\right)$ against FIESTA for several values of the conformal cross ratios.

its of precision, and excellent agreement with the analytic function at 40 different points. See table 4 for an illustrative sample of points.

\section{Conclusions}

Recent years have seen a lot of advances in the analytic computation of Feynman integrals contributing to the perturbative expansion of physical observables. In particular, a more solid understanding of the mathematics underlying the leading singularities and the classes of functions that appear at low loop orders have opened up new ways of evaluating multi-scale multi-loop Feynman integrals analytically.

In this paper we applied some of these new mathematical techniques to the computation of the two so far unknown integrals appearing in the three-loop four-point stress-tensor correlator in $\mathcal{N}=4 \mathrm{SYM}$, and even a first integral occurring in the planar four-loop contribution to the same function. The computation was made possible by postulating that these integrals can be written as a sum over all the leading singularities (defined as the residues at the global poles of the loop integrand), each leading singularity being multiplied by a pure transcendental function that can be written as a $\mathbb{Q}$-linear combination of single-valued multiple polylogarithms in one complex variable. After a suitable choice was made for the entries that can appear in the symbols of these functions, the coefficients can easily be fixed by matching to some asymptotic expansions of the integrals in the limit where one of the cross ratios vanishes. In all cases we were able to integrate the symbols obtained from this procedure to a unique polylogarithmic function, thus completing the analytic computation of the three-loop four-point stress-tensor correlator in $\mathcal{N}=4$ SYM. While for the Easy integral the space of polylogarithmic function is completely classified in the mathematical literature, new classes of multiple polylogarithms appear in the analytic results for the Hard integral and the four-loop integral we considered. 
One might wonder, given that the Hard integral function $H^{(a)}$ involves genuine twovariable functions, whether there could have been a similar contribution to the Easy integral, compatible with all asymptotic limits. Indeed there does exist a symbol of a singlevalued function, not expressible in terms of SVHPLs alone, which evades all constraints from the asymptotic limits. In other words the function is power suppressed in all limits, possibly up to terms proportional to zeta values. However, the evidence we have presented (in particular the numerical checks) strongly suggests that such a contribution is absent and therefore the Easy integral is expressible in terms of SVHPLs only.

We emphasise that the techniques we used for the computation are not limited to the rather special setting of the $\mathcal{N}=4$ model. First, by sending a point to infinity a conformal four-point integral becomes a near generic three-point integral. Such integrals appear as master integrals for phenomenologically relevant processes, like for example the quantum corrections to the decay of a heavy particle into two massive particles. Second, the conformal integrals we calculated have the structure $\sum R_{i} F_{i}$ (so residue times pure function) that is also observed for integrals contributing to on-shell amplitudes. However, we believe that this is in fact a common feature of large classes of Feynman integrals (if not all) and one purpose of this work is to advocate our combination of techniques as a means of solving many other diagrams.

Further increasing the loop-order or the number of points might eventually hamper our prospects of success. Indeed, beyond problems of merely combinatorial nature there are also more fundamental issues, for example to what extent multiple polylogarithms exhaust the function spaces. It is anticipated in ref. $[88,89]$ that elliptic integrals will eventually appear in higher-point on-shell amplitudes. Via the correlator/amplitude duality this observation will eventually carry over to our setting. Nevertheless, some papers $[89,90]$ also hint at a more direct albeit related way of evaluating loop-integrals by casting them into a ' $d$ log-form', which should have a counterpart for off-shell correlators.

\section{Acknowledgments}

We acknowledge stimulating discussions with Simon Caron-Huot. We are grateful to Alexander Smirnov for the possibility to use his c++ version of FIRE. PH would also like to acknowledge many inspirational discussions with Hugh Osborn and Francis Dolan (to whom this work is dedicated) from 2005-6 in which we were attempting to find the threeloop correlator from the corresponding twist two anomalous dimensions using many similar ideas to the current work. CD is supported by the ERC grant 'IterQCD'. BE is supported by the DFG 'eigene Stelle' 32302603. PH is supported by STFC through the Consolidated Grant number ST/J000426/1. JP is supported in part by the US Department of Energy under contract DE-AC02-76SF00515. The work of VS was supported by the Alexander von Humboldt Foundation (Humboldt Forschungspreis) and by the Russian Foundation for Basic Research through grant 11-02-01196. 


\section{A Asymptotic expansions of the Easy and Hard integrals}

In this appendix we collect the asymptotic expansions of the different orientations of the Easy and Hard integrals in terms of harmonic polylogarithms. The results for $E_{14 ; 23}$ and $H_{12 ; 34}$ were already presented in section 3 . The results for the other orientations are given below.

$$
\begin{aligned}
& x_{13}^{2} x_{24}^{2} E_{12 ; 34}=\log ^{3} u\left[-\frac{1}{3 x^{2}}\left(2 H_{1,2}+H_{1,1,1}\right)+\frac{1}{3 x}\left(H_{1,2}+H_{1,1,1}\right)\right] \\
& +\log ^{2} u\left[\frac{2}{x^{2}}\left(2 H_{2,2}+H_{2,1,1}+2 H_{1,3}+H_{1,1,2}\right)\right. \\
& \left.-\frac{1}{2 x}\left(-4 H_{2,2}-3 H_{2,1,1}-4 H_{1,3}-H_{1,2,1}-4 H_{1,1,2}\right)\right] \\
& +\log u\left[\frac { 1 } { x ^ { 2 } } \left(-16 H_{3,2}-8 H_{3,1,1}-16 H_{2,3}-8 H_{2,1,2}-8 H_{1,4}+4 H_{1,3,1}\right.\right. \\
& -4 H_{1,2,2}-H_{1,2,1,1}-4 H_{1,1,3}+2 H_{1,1,2,1}-H_{1,1,1,2} \\
& +\frac{1}{x}\left(8 H_{3,2}+5 H_{3,1,1}+8 H_{2,3}+H_{2,2,1}+6 H_{2,1,2}+4 H_{1,4}-H_{1,3,1}\right. \\
& \left.\left.+5 H_{1,2,2}+H_{1,2,1,1}+4 H_{1,1,3}-2 H_{1,1,2,1}+H_{1,1,1,2}\right)\right] \\
& +\frac{1}{x^{2}}\left(4 \zeta_{3} H_{1,2}+2 \zeta_{3} H_{1,1,1}+32 H_{4,2}+16 H_{4,1,1}+32 H_{3,3}+16 H_{3,1,2}\right. \\
& +16 H_{2,4}-8 H_{2,3,1}+8 H_{2,2,2}+2 H_{2,2,1,1}+8 H_{2,1,3}-4 H_{2,1,2,1}+2 H_{2,1,1,2} \\
& -8 H_{1,4,1}+4 H_{1,3,2}+4 H_{1,3,1,1}+4 H_{1,2,3}-2 H_{1,2,2,1}+2 H_{1,2,1,2}-4 H_{1,1,3,1} \\
& \left.+H_{1,1,2,1,1}-H_{1,1,1,2,1}\right)+\frac{1}{x}\left(-4 \zeta_{3} H_{2,1}-6 \zeta_{3} H_{1,2}-2 \zeta_{3} H_{1,1,1}-16 H_{4,2}\right. \\
& -10 H_{4,1,1}-16 H_{3,3}-10 H_{3,1,2}-8 H_{2,4}+4 H_{2,3,1}-8 H_{2,2,2}-2 H_{2,2,1,1} \\
& -6 H_{2,1,3}+4 H_{2,1,2,1}-2 H_{2,1,1,2}+2 H_{1,4,1}-6 H_{1,3,2}-4 H_{1,3,1,1}-6 H_{1,2,3} \\
& +2 H_{1,2,2,1}-2 H_{1,2,1,2}+4 H_{1,1,3,1}-H_{1,1,2,1,1}+H_{1,1,1,2,1}-8 \zeta_{3} H_{3} \\
& \left.+20 \zeta_{5} H_{1}\right)+\mathcal{O}(u) \\
& x_{13}^{2} x_{24}^{2} E_{13 ; 24}=\frac{\log u}{x}\left(H_{2,2,1}-H_{2,1,2}+H_{1,3,1}-H_{1,2,1,1}-H_{1,1,3}+H_{1,1,2,1}-6 \zeta_{3} H_{2}\right) \\
& +\frac{1}{x}\left(4 \zeta_{3} H_{2,1}-2 \zeta_{3} H_{1,2}-2 H_{3,2,1}+2 H_{3,1,2}-2 H_{2,3,1}+H_{2,2,1,1}+2 H_{2,1,3}\right. \\
& -2 H_{2,1,2,1}+H_{2,1,1,2}-4 H_{1,4,1}+3 H_{1,3,1,1}+H_{1,2,1,2}+4 H_{1,1,4}-2 H_{1,1,3,1} \\
& \left.-H_{1,1,2,2}-H_{1,1,2,1,1}-H_{1,1,1,3}+H_{1,1,1,2,1}+12 \zeta_{3} H_{3}\right)+\mathcal{O}(u), \\
& x_{13}^{4} x_{24}^{4} H_{13 ; 24}=\log ^{3} u\left[\frac{1}{3 x^{2}}\left(2 H_{2,1}-H_{1,2}-H_{1,1,1}\right)+\frac{1}{3(1-x) x}\left(H_{2,1}-H_{3}\right)\right] \\
& +\log ^{2} u\left[\frac{1}{x^{2}}\left(-4 H_{3,1}-2 H_{2,2}+2 H_{1,3}+2 H_{1,2,1}+2 H_{1,1,2}\right)\right. \\
& \left.+\frac{1}{(1-x) x}\left(-2 H_{3,1}-H_{2,2}-H_{2,1,1}-H_{1,3}+H_{1,2,1}+4 H_{4}\right)\right] \\
& +\log u\left[\frac { 1 } { x ^ { 2 } } \left(16 H_{3,2}+8 H_{3,1,1}+8 H_{2,3}-8 H_{2,2,1}-4 H_{1,4}-12 H_{1,3,1}\right.\right. \\
& \left.-4 H_{1,2,2}+2 H_{1,2,1,1}-4 H_{1,1,3}-2 H_{1,1,1,2}\right)+\frac{1}{(1-x) x}\left(4 H_{4,1}+4 H_{3,2}\right.
\end{aligned}
$$




$$
\begin{aligned}
& +6 H_{3,1,1}+4 H_{2,3}+2 H_{2,1,2}+8 H_{1,4}-4 H_{1,3,1}-2 H_{1,2,2}-2 H_{1,2,1,1} \\
& \left.\left.-2 H_{1,1,3}+2 H_{1,1,2,1}-20 H_{5}\right)\right]+\frac{1}{x^{2}}\left(32 \zeta_{3} H_{2,1}-16 \zeta_{3} H_{1,2}-16 \zeta_{3} H_{1,1,1}\right. \\
& +64 H_{5,1}-32 H_{4,2}-32 H_{4,1,1}-24 H_{3,3}+16 H_{3,2,1}-16 H_{3,1,2}-24 H_{2,4} \\
& +40 H_{2,3,1}-4 H_{2,2,1,1}-8 H_{2,1,3}+4 H_{2,1,1,2}+40 H_{1,4,1}+4 H_{1,3,2} \\
& \left.-8 H_{1,3,1,1}-4 H_{1,2,3}+4 H_{1,2,2,1}-4 H_{1,2,1,2}+8 H_{1,1,1,3}\right) \\
& +\frac{1}{(1-x) x}\left(16 \zeta_{3} H_{2,1}-4 H_{4,2}-12 H_{4,1,1}-4 H_{3,3}-12 H_{3,2,1}-8 H_{3,1,2}\right. \\
& -12 H_{2,4}+4 H_{2,3,1}+2 H_{2,2,1,1}-4 H_{2,1,2,1}+2 H_{2,1,1,2}-20 H_{1,5}+4 H_{1,4,1} \\
& +4 H_{1,3,2}+6 H_{1,3,1,1}+4 H_{1,2,3}+2 H_{1,2,1,2}+8 H_{1,1,4}-4 H_{1,1,3,1}-2 H_{1,1,2,2} \\
& \left.-2 H_{1,1,2,1,1}-2 H_{1,1,1,3}+2 H_{1,1,1,2,1}-16 \zeta_{3} H_{3}+40 H_{6}\right)+\mathcal{O}(u), \\
& x_{13}^{4} x_{24}^{4} H_{14 ; 23}=\frac{\log ^{3} u}{3 x}\left[\frac{1}{x}\left(2 H_{2,1}-H_{1,2}+2 H_{1,1,1}\right)-2 H_{2,1}-H_{1,2}-2 H_{1,1,1}-H_{3}\right] \\
& +\log ^{2} u\left[-\frac{2}{x^{2}}\left(2 H_{3,1}+H_{2,2}-H_{1,3}+2 H_{1,2,1}+2 H_{1,1,2}\right)\right. \\
& \left.+\frac{4}{x}\left(H_{3,1}+H_{2,2}+H_{1,3}+H_{1,2,1}+H_{1,1,2}+H_{4}\right)\right] \\
& +\log u\left[\frac { 4 } { x ^ { 2 } } \left(4 H_{3,2}-4 H_{3,1,1}+2 H_{2,3}+4 H_{2,1,2}-H_{1,4}+2 H_{1,3,1}+4 H_{1,2,2}\right.\right. \\
& \left.-2 H_{1,2,1,1}+4 H_{1,1,3}+2 H_{1,1,1,2}\right)+\frac{4}{x}\left(2 H_{4,1}+4 H_{3,2}-2 H_{3,1,1}+4 H_{2,3}\right. \\
& +2 H_{2,1,2}+5 H_{1,4}+2 H_{1,3,1}+4 H_{1,2,2}-2 H_{1,2,1,1}+4 H_{1,1,3}+2 H_{1,1,1,2} \\
& \left.\left.+5 H_{5}\right)\right]+\frac{8}{x^{2}}\left(4 \zeta_{3} H_{2,1}-2 \zeta_{3} H_{1,2}+4 \zeta_{3} H_{1,1,1}+8 H_{5,1}-4 H_{4,2}+8 H_{4,1,1}\right. \\
& -6 H_{3,3}+4 H_{3,2,1}-4 H_{3,1,2}-3 H_{2,4}+2 H_{2,3,1}-4 H_{2,2,2}+2 H_{2,2,1,1} \\
& -6 H_{2,1,3}-2 H_{2,1,1,2}+2 H_{1,4,1}-3 H_{1,3,2}+4 H_{1,3,1,1}-5 H_{1,2,3}+2 H_{1,2,2,1} \\
& \left.-2 H_{1,2,1,2}-4 H_{1,1,4}-2 H_{1,1,2,2}-2 H_{1,1,1,3}\right)+\frac{8}{x}\left(-4 \zeta_{3} H_{2,1}-2 \zeta_{3} H_{1,2}\right. \\
& -4 \zeta_{3} H_{1,1,1}+3 H_{4,2}-2 H_{4,1,1}+3 H_{3,3}-2 H_{3,2,1}+4 H_{2,4}+2 H_{2,2,2} \\
& +2 H_{2,1,3}+5 H_{1,5}+3 H_{1,3,2}-2 H_{1,3,1,1}+3 H_{1,2,3}-2 H_{1,2,2,1}+4 H_{1,1,4} \\
& \left.+2 H_{1,1,2,2}+2 H_{1,1,1,3}-2 \zeta_{3} H_{3}+5 H_{6}\right)+\mathcal{O}(u),
\end{aligned}
$$

\section{B An integral formula for the Hard integral}

We want to find an integral formula for pure functions which involve $x-\bar{x}$ in the symbol as well as $x, \bar{x}, 1-x, 1-\bar{x}$. We are interested in single-valued functions, i.e. ones obeying the constraints on the discontinuities,

$$
\left[\operatorname{disc}_{x}-\operatorname{disc}_{\bar{x}}\right] f(x, \bar{x})=0, \quad\left[\operatorname{disc}_{1-x}-\operatorname{disc}_{1-\bar{x}}\right] f(x, \bar{x})=0 .
$$

and with no other discontinuities. 
It will be sufficient for us to consider functions whose symbols have final letters drawn from a restricted set of letters,

$$
\mathcal{S}(F)=\mathcal{S}(X) \otimes \frac{x}{\bar{x}}+\mathcal{S}(Y) \otimes \frac{1-x}{1-\bar{x}}+\mathcal{S}(Z) \otimes(x-\bar{x}) .
$$

where $X, Y, Z$ are single-valued functions of $x, \bar{x}$.

We will suppose also that the function $F$ obeys $F(x, x)=0$, as required to remove the poles at $x=\bar{x}$ present in the leading singularities of the conformal integrals. We therefore take $Z(x, x)=0$ also. If $F$ has a definite parity under $x \leftrightarrow \bar{x}$ then $X$ and $Y$ have the opposite parity while $Z$ has the same parity.

The functions $X, Y$ and $Z$ are not independent of each other. Integrability (i.e. $d^{2} F=$ $0)$ imposes the following restrictions,

$$
d X \wedge d \log \frac{x}{\bar{x}}+d Y \wedge d \log \frac{1-x}{1-\bar{x}}+d Z \wedge d \log (x-\bar{x})=0 .
$$

We may then define the derivative of $F$ w.r.t. $x$ to be

$$
\partial_{x} F(x, \bar{x})=\frac{X}{x}-\frac{Y}{1-x}+\frac{Z}{x-\bar{x}}
$$

so that

$$
F(x, \bar{x})=\int_{\bar{x}}^{x} d t\left[\frac{X(t, \bar{x})}{t}-\frac{Y(t, \bar{x})}{1-t}+\frac{Z(t, \bar{x})}{t-\bar{x}}\right] .
$$

A trivial example is the Bloch-Wigner dilogarithm function, defined via,

$$
F_{2}(x, \bar{x})=\log x \bar{x}\left(H_{1}(x)-H_{1}(\bar{x})\right)-2\left(H_{2}(x)-H_{2}(\bar{x})\right) .
$$

It has a symbol of the form (B.2) where

$$
X_{1}=\log (1-x)(1-\bar{x}), \quad Y_{1}=-\log x \bar{x} \quad Z_{1}=0 .
$$

Thus we can write the integral formula (B.5) for $F_{2}$.

\section{B.1 Limits}

We want to be able to calculate the limits of the functions to compare with the asymptotic expressions obtained in section 3. The formula (B.5) allows us to calculate the limit $\bar{x} \rightarrow 0$ (which means dropping any power suppressed terms in this limit). We may commute the limit and integration

$$
\lim _{\bar{x} \rightarrow 0} F(x, \bar{x})=\int_{\bar{x}}^{x} d t \lim _{\bar{x} \rightarrow 0}\left[\frac{X(t, \bar{x})}{t}-\frac{Y(t, \bar{x})}{1-t}+\frac{Z(t, \bar{x})}{t}\right] .
$$

In the second and third terms one may also set the lower limit of integration to zero. directly. In the first one should take care that contributions from $X(t, \bar{x})$ which do not vanish as $t \rightarrow 0$ produce extra logarithms of $\bar{x}$, beyond those explicitly appearing in the limit of $X$, as the lower limit approaches zero. 


\section{B.2 First non-trivial example (weight three)}

The first example of a single-valued function whose symbol involves $x-\bar{x}$ is at weight three [58]. There is exactly one such function at this weight, i.e. all single-valued functions can be written in terms of this one and single-valued functions constructed from single-variable HPLs with arguments $x$ and $\bar{x}$ only. It obeys $F_{3}(x, \bar{x})=-F_{3}(\bar{x}, x)$. The symbol takes the form (B.2) with

$$
\begin{aligned}
X_{2} & =-\log (x \bar{x})\left(H_{1}(x)+H_{1}(\bar{x})\right)+\frac{1}{2}\left(H_{1}(x)+H_{1}(\bar{x})\right)^{2}, \\
Y_{2} & =-\frac{1}{2} \log ^{2}(x \bar{x})+\log (x \bar{x})\left(H_{1}(x)+H_{1}(\bar{x})\right), \\
Z_{2} & =2 \log x \bar{x}\left(H_{1}(x)-H_{1}(\bar{x})\right)-4\left(H_{2}(x)-H_{2}(\bar{x})\right) .
\end{aligned}
$$

Note that $X_{2}, Y_{2}$ and $Z_{2}$ are single-valued and that $Z_{2}$ is proportional to the Bloch-Wigner dilogarithm (it is the only antisymmetric weight-two single-valued function so it had to be). They obey the integrability condition (B.3) so we can write the integral formula (B.5) to define the function $F_{3}$.

We have constructed a single-valued function with a given symbol, but in fact this function is uniquely defined since there is no antisymmetric function of weight one which is single-valued which could be multiplied by $\zeta_{2}$ and added to our result. Moreover, since it is antisymmetric in $x$ and $\bar{x}$, we cannot add a constant term proportional to $\zeta_{3}$.

Looking at the limit $\bar{x} \rightarrow 0$ we find, following the discussion above,

$$
\begin{aligned}
\lim _{\bar{x} \rightarrow 0} F_{3}(x, \bar{x})= & \frac{1}{2} \log ^{2} \bar{x} H_{1}(x)+\log \bar{x}\left(H_{2}(x)+H_{1,0}(x)-H_{1,1}(x)\right) \\
& -3 H_{3}(x)-H_{1,2}(x)+H_{2,0}(x)+H_{2,1}(x)+H_{1,0,0}(x)-H_{1,1,0}(x)
\end{aligned}
$$

Starting from the original symbol for $F_{3}$ and taking the limit $\bar{x} \rightarrow 0$ we see that the above formula indeed correctly captures the limit.

\section{B.3 Weight five example}

We now give an example directly analogous to the weight-three example above but at weight five. The example we are interested in is symmetric $F_{5}(x, \bar{x})=F_{5}(\bar{x}, x)$. It has a symbol of the canonical form (B.2) with

$$
\begin{aligned}
X_{4}(x, \bar{x}) & =\left(\mathcal{L}_{0,0,1,1}-\mathcal{L}_{1,1,0,0}-\mathcal{L}_{0,1,1,1}+\mathcal{L}_{1,1,1,0}\right), \\
Y_{4}(x, \bar{x}) & =\left(\mathcal{L}_{0,0,0,1}-\mathcal{L}_{1,0,0,0}-\mathcal{L}_{0,0,1,1}+\mathcal{L}_{1,1,0,0}\right), \\
Z_{4}(x, \bar{x}) & =\left(\mathcal{L}_{0,0,1,1}+\mathcal{L}_{1,1,0,0}-\mathcal{L}_{0,1,1,0}-\mathcal{L}_{1,0,0,1}\right) .
\end{aligned}
$$

The above functions are single-valued and obey the integrability condition and therefore define a single-valued function of two variables of weight five via the integral formula.

Taking the limit $\bar{x} \rightarrow 0$ we find

$$
\begin{aligned}
\lim _{\bar{x} \rightarrow 0} F_{5}(x, \bar{x})= & H_{1,1} \bar{H}_{0,0,0}+\left(H_{1,1,0}-H_{1,1,1}\right) \bar{H}_{0,0} \\
& +\left(-H_{3,1}+H_{2,1,1}+H_{1,1,0,0}-H_{1,1,1,0}\right) \bar{H}_{0}
\end{aligned}
$$




$$
\begin{aligned}
& -H_{1,4}-H_{2,3}+2 H_{4,1}+H_{1,3,1}-H_{3,1,0}-H_{3,1,1} \\
& +H_{2,1,1,0}+H_{1,1,0,0,0}-H_{1,1,1,0,0}+2 H_{1,1} \zeta_{3} .
\end{aligned}
$$

This formula correctly captures the limit taken directly on the symbol of $F_{5}$. This weightfive function plays a role in the construction of the Hard integral.

\section{B.4 The function $H^{(a)}$ from the Hard integral}

The function $H^{(a)}$ from the Hard integral is a weight-six symmetric function obeying the condition $H^{(a)}(x, x)=0$. The symbol of $H^{(a)}$ is known but is not of the form (B.2). However, we can use shuffle relations to rewrite the symbol in terms of logarithms of $u$ and $v$ and functions which end with our preferred set of letters. We find the symbol can be represented by a function of the form

$$
\begin{aligned}
H^{(a)}(1 & -x, 1-\bar{x}) \\
= & \left(2 H_{0,0}(u)+4 H_{0}(u) H_{0}(v)+8 H_{0,0}(v)\right)\left(\mathcal{L}_{0,0,1,1}+\mathcal{L}_{1,1,0,0}-\mathcal{L}_{0,1,1,0}-\mathcal{L}_{1,0,0,1}\right) \\
& -8 F_{5}\left(H_{0}(u)+2 H_{0}(v)\right)+F_{6} .
\end{aligned}
$$

Here $F_{5}$ is the weight-five function defined in section B.3. The function $F_{6}$ is now one whose symbol is of the form (B.2), where the functions $X_{5}, Y_{5}$ and $Z_{5}$ take the form

$$
\begin{aligned}
X_{5}= & 20 \mathcal{L}_{0,0,0,1,1}+12 \mathcal{L}_{0,0,1,1,0}-32 \mathcal{L}_{0,0,1,1,1}-8 \mathcal{L}_{0,1,0,1,1}-12 \mathcal{L}_{0,1,1,0,0}-8 \mathcal{L}_{0,1,1,0,1} \\
& +16 \mathcal{L}_{0,1,1,1,1}-8 \mathcal{L}_{1,0,0,1,1}+8 \mathcal{L}_{1,0,1,1,0}-20 \mathcal{L}_{1,1,0,0,0}+8 \mathcal{L}_{1,1,0,0,1}+8 \mathcal{L}_{1,1,0,1,0} \\
& +32 \mathcal{L}_{1,1,1,0,0}-16 \mathcal{L}_{1,1,1,1,0}-16 \mathcal{L}_{1,1} \zeta_{3} \\
Y_{5}= & 20 \mathcal{L}_{0,0,0,0,1}-32 \mathcal{L}_{0,0,0,1,1}-8 \mathcal{L}_{0,0,1,1,0}+16 \mathcal{L}_{0,0,1,1,1}-8 \mathcal{L}_{0,1,0,0,1}+\mathcal{L}_{0,1,1,0,0} \\
& -20 \mathcal{L}_{1,0,0,0,0}+8 \mathcal{L}_{1,0,0,1,0}+16 \mathcal{L}_{1,0,0,1,1}+8 \mathcal{L}_{1,0,1,0,0}+32 \mathcal{L}_{1,1,0,0,0}-16 \mathcal{L}_{1,1,0,0,1} \\
& -16 \mathcal{L}_{1,1,1,0,0}-16 \mathcal{L}_{1,0} \zeta_{3}+64 \mathcal{L}_{1,1} \zeta_{3} . \\
Z_{5}= & 32 F_{5} .
\end{aligned}
$$

Note that the $\zeta_{3}$ terms have been chosen in such a way the functions $X_{5}, Y_{5}$ and $Z_{5}$ obey the integrability condition (B.3). The integral formula for $F_{6}$ based on the above functions will give a single-valued function with the correct symbol, i.e. one such that $H^{(a)}$ defined in eq. (B.13) has the correct symbol and is single-valued.

We recall that the Hard integral takes the form

$$
H_{14 ; 23}=\frac{1}{x_{13}^{4} x_{24}^{4}}\left[\frac{H^{(a)}(1-x, 1-\bar{x})}{(x-\bar{x})^{2}}+\frac{H^{(b)}(1-x, 1-\bar{x})}{(1-x \bar{x})(x-\bar{x})}\right] .
$$

Calculating the limit $\bar{x} \rightarrow 0$ we find that $H^{(a)}$ reproduces the terms proportional to $1 / x^{2}$ in the limit exactly, including the zeta terms. Note that in this limit the contributions of $H^{(a)}$ and $H^{(b)}$ are distinguishable since the harmonic polylogarithms come with different powers of $x$. Since there are no functions of weight four or lower which are symmetric in $x$ and $\bar{x}$ and which vanish at $x=\bar{x}$ and which vanish in the limit $\bar{x} \rightarrow 0$, we conclude that $H^{(a)}$ defined in eq. (B.13) is indeed the function. Comparing numerically with the formula obtained in section 5 we indeed find agreement to at least five significant figures. 


\section{A symbol-level solution of the four-loop differential equation}

In this appendix we sketch an alternative approach to the evaluation of the four-loop integral. More precisely, we will show how the function $I^{(4)}$ can be determined using symbols and the coproduct on multiple polylogarithms. We start from the differential equation (7.17), which we recall here for convenience,

$$
\partial_{x} \partial_{\bar{x}} \hat{f}(x, \bar{x})=-\frac{1}{(1-x \bar{x}) x \bar{x}} E_{1}(x, \bar{x})-\frac{1}{(1-x \bar{x})} E_{2}(x, \bar{x}),
$$

where we used the abbreviations $E_{1}(x, \bar{x})=E(1-x, 1-\bar{x})$ and $E_{2}(x, \bar{x})=E(1-1 / x, 1-$ $1 / \bar{x})$. We now act with the symbol map $\mathcal{S}$ on the differential equation, and we get

$$
\partial_{x} \partial_{\bar{x}} \mathcal{S}[\hat{f}(x, \bar{x})]=-\frac{1}{(1-x \bar{x}) x \bar{x}} \mathcal{S}\left[E_{1}(x, \bar{x})\right]-\frac{1}{(1-x \bar{x})} \mathcal{S}\left[E_{2}(x, \bar{x})\right],
$$

where the differential operators act on tensors only in the last entry, e.g.,

$$
\partial_{x}\left[a_{1} \otimes \ldots \otimes a_{n}\right]=\left[\partial_{x} \log a_{n}\right] a_{1} \otimes \ldots \otimes a_{n-1},
$$

and similarly for $\partial_{\bar{x}}$. It is easy to see that the tensor

$$
S_{1}=\mathcal{S}\left[E_{1}(x, \bar{x})\right] \otimes\left(1-\frac{1}{x \bar{x}}\right) \otimes(x \bar{x})+\mathcal{S}\left[E_{2}(x, \bar{x})\right] \otimes(1-x \bar{x}) \otimes(x \bar{x})
$$

solves the equation (C.2). However, $S_{1}$ is not integrable in the pair of entries $(6,7)$, and so $S_{1}$ is not yet the symbol of a solution of the differential equation. In order to obtain an integrable solution, we need to add a solution to the homogeneous equation associated to eq. (C.2). The homogeneous solution can easily be obtained by writing down the most general tensor $S_{2}$ with entries drawn from the set $\{x, \bar{x}, 1-x, 1-\bar{x}, 1-x \bar{x}\}$ that has the correct symmetries and satisfies the first entry condition and

$$
\partial_{x} \partial_{\bar{x}} S_{2}=0
$$

In addition, we may assume that $S_{2}$ satisfies the integrability condition in all factors of the tensor product except for the pair of entries $(6,7)$, because $S_{1}$ satisfies this condition as well. The symbol of the solution of the differential equation is then given by $S_{1}+S_{2}$, subject to the constraint that the sum is integrable. It turns out that there is a unique solution, which can be written in the schematic form

$$
\begin{aligned}
\mathcal{S}[\hat{f}(x, \bar{x})] & =s_{1}^{-} \otimes u \otimes u+s_{2}^{-} \otimes v \otimes u+s_{3}^{-} \otimes \frac{1-x}{1-\bar{x}} \otimes \frac{x}{\bar{x}}+s_{4}^{+} \otimes \frac{x}{\bar{x}} \otimes u \\
& +s_{5}^{+} \otimes u \otimes \frac{x}{\bar{x}}+s_{6}^{+} \otimes \frac{1-x}{1-\bar{x}} \otimes u+s_{7}^{+} \otimes v \otimes \frac{x}{\bar{x}}+s_{8}^{-} \otimes \frac{x}{\bar{x}} \otimes \frac{x}{\bar{x}} \\
& +s_{9}^{-} \otimes(1-u) \otimes u,
\end{aligned}
$$

where $s_{i}^{ \pm}$are (integrable) tensor that have all their entries drawn from the set $\{x, \bar{x}, 1-x, 1-\bar{x}\}$ and the superscript refers to the parity under an exchange of $x$ and $\bar{x}$. 
The form (C.6) of the symbol of $\hat{f}(x, \bar{x})$ allows us to make the following more refined ansatz: as the $s_{i}^{ \pm}$are symbols of SVHPLs, and using the fact that the symbol is the maximal iteration of the coproduct, we conclude that there are linear combinations $f_{i}^{ \pm}(x, \bar{x})$ of SVHPLs of weight six (including products of zeta values and SVHPLs of lower weight) such that $\mathcal{S}\left[f_{i}^{ \pm}(x, \bar{x})\right]=s_{i}^{ \pm}$and

$$
\begin{aligned}
\Delta_{6,1,1}[\hat{f}(x, \bar{x})] & =f_{1}^{-}(x, \bar{x}) \otimes \log u \otimes \log u+f_{2}^{-}(x, \bar{x}) \otimes \log v \otimes \log u \\
& +f_{3}^{-}(x, \bar{x}) \otimes \log \frac{1-x}{1-\bar{x}} \otimes \log \frac{x}{\bar{x}}+f_{4}^{+}(x, \bar{x}) \otimes \log \frac{x}{\bar{x}} \otimes \log u \\
& +f_{5}^{+}(x, \bar{x}) \otimes \log u \otimes \log \frac{x}{\bar{x}}+f_{6}^{+}(x, \bar{x}) \otimes \log \frac{1-x}{1-\bar{x}} \otimes \log u \\
& +f_{7}^{+}(x, \bar{x}) \otimes \log v \otimes \log \frac{x}{\bar{x}}+f_{8}^{-}(x, \bar{x}) \otimes \log \frac{x}{\bar{x}} \otimes \log \frac{x}{\bar{x}} \\
& +f_{9}^{-}(x, \bar{x}) \otimes \log (1-u) \otimes u .
\end{aligned}
$$

The coefficients of the terms proportional to zeta values and SVHPLs of lower weight (which were not captured by the symbol) can easy be fixed by appealing to the differential equation, written in the form ${ }^{10}$

$$
\left(\mathrm{id} \otimes \partial_{x} \otimes \partial_{\bar{x}}\right) \Delta_{6,1,1}[\hat{f}(x, \bar{x})]=-\frac{1}{(1-x \bar{x}) x \bar{x}} E_{1}(x, \bar{x}) \otimes 1 \otimes 1-\frac{1}{(1-x \bar{x})} E_{2}(x, \bar{x}) \otimes 1 \otimes 1 .
$$

The expression (C.7) has the advantage that it captures more information about the function $\hat{f}(x, \bar{x})$ than the symbol alone. In particular, we can use eq. (C.7) to derive an iterated integral representation for $\hat{f}(x, \bar{x})$ with respect to $x$ only. To see how this works, first note that there must be functions $A^{ \pm}(x, \bar{x})$, that are respectively even and odd under an exchange of $x$ and $\bar{x}$, such that

$$
\Delta_{7,1}[\hat{f}(x, \bar{x})]=A^{-}(x, \bar{x}) \otimes \log u+A^{+}(x, \bar{x}) \otimes \log \frac{x}{\bar{x}} .
$$

with

$$
\begin{aligned}
\Delta_{6,1}\left[A^{-}(x, \bar{x})\right] & =f_{1}^{-}(x, \bar{x}) \otimes \log u+f_{2}^{-}(x, \bar{x}) \otimes \log v+f_{4}^{+}(x, \bar{x}) \otimes \log \frac{x}{\bar{x}} \\
& +f_{6}^{+}(x, \bar{x}) \otimes \log \frac{1-x}{1-\bar{x}}+f_{9}^{-}(x, \bar{x}) \otimes \log (1-u), \\
\Delta_{6,1}\left[A^{+}(x, \bar{x})\right] & =f_{3}^{-}(x, \bar{x}) \otimes \log \frac{1-x}{1-\bar{x}}+f_{5}^{+}(x, \bar{x}) \otimes \log u \\
& +f_{7}^{+}(x, \bar{x}) \otimes \log v+f_{8}^{-}(x, \bar{x}) \otimes \log \frac{x}{\bar{x}} .
\end{aligned}
$$

The $(6,1)$ component of the coproduct of $A^{+}(x, \bar{x})$ does not involve $\log (1-u)$, and so it can entirely be expressed in terms of SVHPLs. We can thus easily obtain the result for $A^{+}(x, \bar{x})$ by writing down the most general linear combination of SVHPLs of weight seven that are even under an exchange of $x$ and $\bar{x}$ and fix the coefficients by requiring the $(6,1)$ component of the coproduct of the linear combination to agree with eq. (C.10). In this

\footnotetext{
${ }^{10}$ We stress that differential operators act in the last factor of the coproduct, just like for the symbol.
} 
way we can fix $A^{+}(x, \bar{x})$ up to zeta values of weight seven (which are integration constants of the original differential equation).

The coproduct of $A^{-}(x, \bar{x})$, however, does involve $\log (1-u)$, and so it cannot be expressed in terms of SVHPLs alone. We can nevertheless derive a first-order differential equation for $A^{-}(x, \bar{x})$. We find

$$
\begin{aligned}
\partial_{x} A^{-}(x, \bar{x}) & =\frac{1}{x}\left[f_{1}^{-}(x, \bar{x})+f_{4}^{+}(x, \bar{x})\right]-\frac{1}{1-x}\left[f_{2}^{-}(x, \bar{x})+f_{6}^{+}(x, \bar{x})\right] \\
& -\frac{\bar{x}}{1-x \bar{x}} f_{9}^{-}(x, \bar{x}) \\
& \equiv K(x, \bar{x}) .
\end{aligned}
$$

The solution to this equation is

$$
A^{-}(x, \bar{x})=h(\bar{x})+\int_{\bar{x}}^{x} d t K(t, \bar{x}),
$$

where $h(\bar{x})$ is an arbitrary function of $\bar{x}$. The integral can easily be performed in terms of multiple polylogarithms. Antisymmetry of $A^{-}(x, \bar{x})$ under an exchange of $x$ and $\bar{x}$ requires $h(\bar{x})$ to vanish identically, because

$$
A^{-}(x, \bar{x})=h(\bar{x})+\int_{\bar{x}}^{x} d t \partial_{t} A^{-}(t, \bar{x})=h(\bar{x})+A^{-}(x, \bar{x})-A^{-}(\bar{x}, \bar{x})=h(\bar{x})+A^{-}(x, \bar{x}) .
$$

We thus obtain a unique solution for $A^{-}(x, \bar{x})$.

Having obtained the analytic expressions for $A^{ \pm}(x, \bar{x})$ (up to the integration constants in $A^{+}(x, \bar{x})$ ), we can easily obtain a first-order differential equation for $\hat{f}(x, \bar{x})$,

$$
\partial_{x} \hat{f}(x, \bar{x})=\frac{1}{x}\left[A^{-}(x, \bar{x})+A^{+}(x, \bar{x})\right] .
$$

The solution reads

$$
\hat{f}(x, \bar{x})=\int_{\bar{x}}^{x} \frac{d t}{t}\left[A^{-}(t, \bar{x})+A^{+}(t, \bar{x})\right] .
$$

The integral can again easily be performed in terms of multiple polylogarithms and the antisymmetry of $\hat{f}(x, \bar{x})$ under an exchange of $x$ and $\bar{x}$ again excludes any arbitrary function of $\bar{x}$ only. The solution to eq. (C.14) is however not yet unique, because of the integration constants in $A^{+}(x, \bar{x})$, and we are left with three free coefficients of the form,

$$
\left(c_{1} \zeta_{7}+c_{2} \zeta_{5} \zeta_{2}+c_{3} \zeta_{4} \zeta_{3}\right) \log \frac{x}{\bar{x}} .
$$

The free coefficients can be fixed using the requirement that $\hat{f}(x, \bar{x})$ be single-valued (see the discussion in section 7$)$. Alternatively, they can be fixed by requiring that $\hat{f}(x, \bar{x})$ be odd under inversion of $(x, \bar{x})$ and vanish at $x=\bar{x}$. We checked that the resulting function agrees analytically with the result derived in section 7 .

Open Access. This article is distributed under the terms of the Creative Commons Attribution License which permits any use, distribution and reproduction in any medium, provided the original author(s) and source are credited. 


\section{References}

[1] M.T. Grisaru, M. Roček and W. Siegel, Zero three loop $\beta$-function in $N=4$ super Yang-Mills theory, Phys. Rev. Lett. 45 (1980) 1063 [INSPIRE].

[2] W.E. Caswell and D. Zanon, Vanishing three loop $\beta$-function in $N=4$ supersymmetric Yang-Mills theory, Phys. Lett. B 100 (1981) 152 [INSPIRE].

[3] P.S. Howe, K. Stelle and P. Townsend, The relaxed hypermultiplet: an unconstrained $N=2$ superfield theory, Nucl. Phys. B 214 (1983) 519 [INSPIRE].

[4] S. Mandelstam, Light cone superspace and the vanishing of the $\beta$-function for the $N=4$ model, in Proc. $21^{\text {st }}$ Int. Conf. on High Energy Physics, P. Petiau and M. Proneuf eds., J. Phys. (France) 43 (1982) C-3 [inSPIRE].

[5] S. Mandelstam, Light cone superspace and the ultraviolet finiteness of the $N=4$ model, Nucl. Phys. B 213 (1983) 149 [inSPIRE].

[6] L. Brink, O. Lindgren and B.E. Nilsson, $N=4$ Yang-Mills theory on the light cone, Nucl. Phys. B 212 (1983) 401 [inSPIRE].

[7] L. Brink, O. Lindgren and B.E. Nilsson, The ultraviolet finiteness of the $N=4$ Yang-Mills theory, Phys. Lett. B 123 (1983) 323 [InSPIRE].

[8] P.S. Howe, K. Stelle and P. Townsend, Miraculous ultraviolet cancellations in supersymmetry made manifest, Nucl. Phys. B 236 (1984) 125 [INSPIRE].

[9] J. Minahan and K. Zarembo, The Bethe ansatz for $N=4$ super Yang-Mills, JHEP 03 (2003) 013 [hep-th/0212208] [INSPIRE].

[10] N. Beisert, V. Dippel and M. Staudacher, A novel long range spin chain and planar $N=4$ super Yang-Mills, JHEP 07 (2004) 075 [hep-th/0405001] [INSPIRE].

[11] N. Beisert and M. Staudacher, Long-range PSU(2,2|4) Bethe ansätze for gauge theory and strings, Nucl. Phys. B 727 (2005) 1 [hep-th/0504190] [INSPIRE].

[12] J.M. Maldacena, The large- $N$ limit of superconformal field theories and supergravity, Adv. Theor. Math. Phys. 2 (1998) 231 [Int. J. Theor. Phys. 38 (1999) 1113] [hep-th/9711200] [INSPIRE].

[13] S. Gubser, I.R. Klebanov and A.M. Polyakov, Gauge theory correlators from noncritical string theory, Phys. Lett. B 428 (1998) 105 [hep-th/9802109] [INSPIRE].

[14] E. Witten, Anti-de Sitter space and holography, Adv. Theor. Math. Phys. 2 (1998) 253 [hep-th/9802150] [INSPIRE].

[15] L.F. Alday and J.M. Maldacena, Gluon scattering amplitudes at strong coupling, JHEP 06 (2007) 064 [arXiv:0705.0303] [INSPIRE].

[16] G. Korchemsky, J. Drummond and E. Sokatchev, Conformal properties of four-gluon planar amplitudes and Wilson loops, Nucl. Phys. B 795 (2008) 385 [arXiv:0707.0243] [INSPIRE].

[17] A. Brandhuber, P. Heslop and G. Travaglini, $M H V$ amplitudes in $N=4$ super Yang-Mills and Wilson loops, Nucl. Phys. B 794 (2008) 231 [arXiv:0707.1153] [INSPIRE].

[18] J. Drummond, J. Henn, G. Korchemsky and E. Sokatchev, Hexagon Wilson loop = six-gluon MHV amplitude, Nucl. Phys. B 815 (2009) 142 [arXiv:0803.1466] [INSPIRE].

[19] Z. Bern et al., The two-loop six-gluon MHV amplitude in maximally supersymmetric Yang-Mills theory, Phys. Rev. D 78 (2008) 045007 [arXiv: 0803.1465] [InSPIRE]. 
[20] G. Korchemsky and A. Radyushkin, Loop space formalism and renormalization group for the infrared asymptotics of QCD, Phys. Lett. B 171 (1986) 459 [INSPIRE].

[21] S. Ivanov, G. Korchemsky and A. Radyushkin, Infrared asymptotics of perturbative QCD: contour gauges, Yad. Fiz. 44 (1986) 230 [Sov. J. Nucl. Phys. 44 (1986) 145] [INSPIRE].

[22] G. Korchemsky and A. Radyushkin, Infrared asymptotics of perturbative QCD. Quark and gluon propagators, Yad. Fiz. 45 (1987) 198 [Sov. J. Nucl. Phys. 45 (1987) 127] [INSPIRE].

[23] G. Korchemsky and A. Radyushkin, Infrared asymptotics of perturbative QCD. Vertex functions, Yad. Fiz. 45 (1987) 1466 [Sov. J. Nucl. Phys. 45 (1987) 910] [InSPIRE].

[24] G. Korchemsky and A. Radyushkin, Renormalization of the Wilson loops beyond the leading order, Nucl. Phys. B 283 (1987) 342 [INSPIRE].

[25] G. Korchemsky and G. Marchesini, Structure function for large $x$ and renormalization of Wilson loop, Nucl. Phys. B 406 (1993) 225 [hep-ph/9210281] [INSPIRE].

[26] L.F. Alday, B. Eden, G.P. Korchemsky, J. Maldacena and E. Sokatchev, From correlation functions to Wilson loops, JHEP 09 (2011) 123 [arXiv: 1007.3243] [INSPIRE].

[27] B. Eden, G.P. Korchemsky and E. Sokatchev, From correlation functions to scattering amplitudes, JHEP 12 (2011) 002 [arXiv: 1007.3246] [INSPIRE].

[28] B. Eden, G.P. Korchemsky and E. Sokatchev, More on the duality correlators/amplitudes, Phys. Lett. B 709 (2012) 247 [arXiv: 1009.2488] [INSPIRE].

[29] E. D'Hoker, D.Z. Freedman, S.D. Mathur, A. Matusis and L. Rastelli, Graviton exchange and complete four point functions in the AdS/CFT correspondence,

Nucl. Phys. B 562 (1999) 353 [hep-th/9903196] [InSPIRE].

[30] E. D'Hoker, S.D. Mathur, A. Matusis and L. Rastelli, The operator product expansion of $N=4 S Y M$ and the 4 point functions of supergravity, Nucl. Phys. B 589 (2000) 38 [hep-th/9911222] [INSPIRE].

[31] G. Arutyunov and S. Frolov, Four point functions of lowest weight CPOs in $N=4 \mathrm{SYM}_{4}$ in supergravity approximation, Phys. Rev. D 62 (2000) 064016 [hep-th/0002170] [INSPIRE].

[32] G. Arutyunov, F. Dolan, H. Osborn and E. Sokatchev, Correlation functions and massive Kaluza-Klein modes in the AdS/CFT correspondence, Nucl. Phys. B 665 (2003) 273 [hep-th/0212116] [INSPIRE].

[33] B. Eden, P.S. Howe, C. Schubert, E. Sokatchev and P.C. West, Four point functions in $N=4$ supersymmetric Yang-Mills theory at two loops, Nucl. Phys. B 557 (1999) 355 [hep-th/9811172] [INSPIRE].

[34] B. Eden, P.S. Howe, C. Schubert, E. Sokatchev and P.C. West, Simplifications of four point functions in $N=4$ supersymmetric Yang-Mills theory at two loops,

Phys. Lett. B 466 (1999) 20 [hep-th/9906051] [INSPIRE].

[35] F. Gonzalez-Rey, I. Park and K. Schalm, A note on four point functions of conformal operators in $N=4$ super Yang-Mills, Phys. Lett. B 448 (1999) 37 [hep-th/9811155] [INSPIRE].

[36] B. Eden, C. Schubert and E. Sokatchev, Three loop four point correlator in $N=4 S Y M$, Phys. Lett. B 482 (2000) 309 [hep-th/0003096] [INSPIRE].

[37] M. Bianchi, S. Kovacs, G. Rossi and Y.S. Stanev, Anomalous dimensions in N $=4$ SYM theory at order $g^{4}$, Nucl. Phys. B 584 (2000) 216 [hep-th/0003203] [INSPIRE]. 
[38] A. Galperin, E. Ivanov, S. Kalitsyn, V. Ogievetsky and E. Sokatchev, Unconstrained $N=2$ matter, Yang-Mills and supergravity theories in harmonic superspace, Class. Quant. Grav. 1 (1984) 469 [InSPIRE].

[39] G. Hartwell and P.S. Howe, $(N, p, q)$ harmonic superspace, Int. J. Mod. Phys. A 10 (1995) 3901 [hep-th/9412147] [INSPIRE].

[40] B. Eden, A.C. Petkou, C. Schubert and E. Sokatchev, Partial nonrenormalization of the stress tensor four point function in $N=4 S Y M$ and $A d S / C F T$, Nucl. Phys. B 607 (2001) 191 [hep-th/0009106] [INSPIRE].

[41] B. Eden, P.S. Howe, A. Pickering, E. Sokatchev and P.C. West, Four point functions in $N=2$ superconformal field theories, Nucl. Phys. B 581 (2000) 523 [hep-th/0001138] [INSPIRE].

[42] F. Dolan and H. Osborn, Superconformal symmetry, correlation functions and the operator product expansion, Nucl. Phys. B 629 (2002) 3 [hep-th/0112251] [INSPIRE].

[43] P. Heslop and P. Howe, Four point functions in N=4 SYM, JHEP 01 (2003) 043 [hep-th/0211252] [INSPIRE].

[44] B. Eden, P. Heslop, G.P. Korchemsky and E. Sokatchev, Hidden symmetry of four-point correlation functions and amplitudes in $N=4$ SYM, Nucl. Phys. B 862 (2012) 193 [arXiv: 1108.3557] [INSPIRE].

[45] B. Eden, P. Heslop, G.P. Korchemsky and E. Sokatchev, Constructing the correlation function of four stress-tensor multiplets and the four-particle amplitude in $N=4 S Y M$, Nucl. Phys. B 862 (2012) 450 [arXiv:1201.5329] [INSPIRE].

[46] Z. Bern, L.J. Dixon and V.A. Smirnov, Iteration of planar amplitudes in maximally supersymmetric Yang-Mills theory at three loops and beyond, Phys. Rev. D 72 (2005) 085001 [hep-th/0505205] [INSPIRE].

[47] A.B. Goncharov, Multiple polylogarithms, cyclotomy and modular complexes, Math. Res. Lett. 5 (1998) 497 [arXiv:1105.2076] [INSPIRE].

[48] F. Cachazo, Sharpening the leading singularity, arXiv:0803.1988 [INSPIRE].

[49] B. Eden, P. Heslop, G.P. Korchemsky, V.A. Smirnov and E. Sokatchev, Five-loop Konishi in $N=4$ SYM, Nucl. Phys. B 862 (2012) 123 [arXiv:1202.5733] [INSPIRE].

[50] B. Eden, Three-loop universal structure constants in $N=4$ SUSY Yang-Mills theory, arXiv:1207.3112 [INSPIRE].

[51] V.A. Smirnov, Applied asymptotic expansions in momenta and masses, Springer Tracts Mod. Phys. 177 (2002) 1 [INSPIRE].

[52] J. Drummond, J. Henn, V. Smirnov and E. Sokatchev, Magic identities for conformal four-point integrals, JHEP 01 (2007) 064 [hep-th/0607160] [INSPIRE].

[53] N. Usyukina and A.I. Davydychev, An approach to the evaluation of three and four point ladder diagrams, Phys. Lett. B 298 (1993) 363 [INSPIRE].

[54] N. Usyukina and A.I. Davydychev, Exact results for three and four point ladder diagrams with an arbitrary number of rungs, Phys. Lett. B 305 (1993) 136 [INSPIRE].

[55] F.C.S. Brown, Single-valued multiple polylogarithms in one variable Comptes Rendus Math. 338 (2004) 527. 
[56] N. Arkani-Hamed, J.L. Bourjaily, F. Cachazo and J. Trnka, Local integrals for planar scattering amplitudes, JHEP 06 (2012) 125 [arXiv:1012.6032] [INSPIRE].

[57] S. Caron-Huot, Loops in spacetime, in ECT*, Trento workshop - Scattering amplitudes: from QCD to maximally supersymmetric Yang-Mills theory and back, http://sites.google.com/site/trentoworkshop/program/, Trento Italy July 16-20 2012.

[58] F. Chavez and C. Duhr, Three-mass triangle integrals and single-valued polylogarithms, JHEP 11 (2012) 114 [arXiv: 1209.2722] [INSPIRE].

[59] A.B. Goncharov, M. Spradlin, C. Vergu and A. Volovich, Classical polylogarithms for amplitudes and Wilson loops, Phys. Rev. Lett. 105 (2010) 151605 [arXiv:1006.5703] [INSPIRE].

[60] D. Gaiotto, J. Maldacena, A. Sever and P. Vieira, Pulling the straps of polygons, JHEP 12 (2011) 011 [arXiv:1102.0062] [INSPIRE].

[61] E. Remiddi and J. Vermaseren, Harmonic polylogarithms, Int. J. Mod. Phys. A 15 (2000) 725 [hep-ph/9905237] [INSPIRE].

[62] J. Fleischer, A. Kotikov and O. Veretin, Analytic two loop results for selfenergy type and vertex type diagrams with one nonzero mass, Nucl. Phys. B 547 (1999) 343 [hep-ph/9808242] [INSPIRE].

[63] J. Drummond, Generalised ladders and single-valued polylogarithms, JHEP 02 (2013) 092 [arXiv: 1207.3824] [INSPIRE].

[64] M. Beneke and V.A. Smirnov, Asymptotic expansion of Feynman integrals near threshold, Nucl. Phys. B 522 (1998) 321 [hep-ph/9711391] [INSPIRE].

[65] V.A. Smirnov, Analytic tools for Feynman integrals, Springer Tracts Mod. Phys. 250 (2012) 1 [INSPIRE].

[66] A. Pak and A. Smirnov, Geometric approach to asymptotic expansion of Feynman integrals, Eur. Phys. J. C 71 (2011) 1626 [arXiv:1011.4863] [INSPIRE].

[67] B. Jantzen, A.V. Smirnov and V.A. Smirnov, Expansion by regions: revealing potential and Glauber regions automatically, Eur. Phys. J. C 72 (2012) 2139 [arXiv:1206.0546] [INSPIRE].

[68] A. Smirnov, Algorithm FIRE - Feynman Integral REduction, JHEP 10 (2008) 107 [arXiv:0807.3243] [INSPIRE].

[69] S. Gorishnii, S. Larin, L. Surguladze and F. Tkachov, MINCER: program for multiloop calculations in quantum field theory for the Schoonschip system, Comput. Phys. Commun. 55 (1989) 381 [INSPIRE].

[70] J. Vermaseren, Harmonic sums, Mellin transforms and integrals, Int. J. Mod. Phys. A 14 (1999) 2037 [hep-ph/9806280] [INSPIRE].

[71] S. Moch, P. Uwer and S. Weinzierl, Nested sums, expansion of transcendental functions and multiscale multiloop integrals, J. Math. Phys. 43 (2002) 3363 [hep-ph/0110083] [InSPIRE].

[72] E.I. Buchbinder and F. Cachazo, Two-loop amplitudes of gluons and octa-cuts in $N=4$ super Yang-Mills, JHEP 11 (2005) 036 [hep-th/0506126] [INSPIRE].

[73] C.W. Bauer, A. Frink and R. Kreckel, Introduction to the GiNaC framework for symbolic computation within the $\mathrm{C}++$ programming language, cs/0004015 [INSPIRE]. 
[74] D. Maître, HPL, a Mathematica implementation of the harmonic polylogarithms, Comput. Phys. Commun. 174 (2006) 222 [hep-ph/0507152] [INSPIRE].

[75] D. Maître, Extension of HPL to complex arguments, Comput. Phys. Commun. 183 (2012) 846 [hep-ph/0703052] [inSPIRE].

[76] A. Smirnov and M. Tentyukov, Feynman Integral Evaluation by a Sector decomposiTion Approach (FIESTA), Comput. Phys. Commun. 180 (2009) 735 [arXiv:0807.4129] [INSPIRE].

[77] A. Smirnov, V. Smirnov and M. Tentyukov, FIESTA 2: parallelizeable multiloop numerical calculations, Comput. Phys. Commun. 182 (2011) 790 [arXiv:0912.0158] [INSPIRE].

[78] O. Schnetz, Graphical functions and single-valued multiple polylogarithms, arXiv:1302.6445 [INSPIRE].

[79] D.E. Radford, A natural ring basis for the shuffle algebra and an application to group schemes, J. Alg. 58 (1979) 432.

[80] A.B. Goncharov, Multiple polylogarithms and mixed Tate motives, math.AG/0103059.

[81] F. Brown, On the decomposition of motivic multiple zeta values, arXiv:1102.1310 [INSPIRE].

[82] C. Duhr, Hopf algebras, coproducts and symbols: an application to Higgs boson amplitudes, JHEP 08 (2012) 043 [arXiv: 1203.0454] [INSPIRE].

[83] P. Baikov and K. Chetyrkin, Four loop massless propagators: an algebraic evaluation of all master integrals, Nucl. Phys. B 837 (2010) 186 [arXiv:1004.1153] [INSPIRE].

[84] R. Lee, A. Smirnov and V. Smirnov, Master integrals for four-loop massless propagators up to transcendentality weight twelve, Nucl. Phys. B 856 (2012) 95 [arXiv:1108.0732] [InSPIRE].

[85] R. Lee, Presenting LiteRed: a tool for the Loop InTEgrals REDuction, arXiv:1212.2685 [INSPIRE].

[86] R. Lee, Group structure of the integration-by-part identities and its application to the reduction of multiloop integrals, JHEP 07 (2008) 031 [arXiv:0804.3008] [INSPIRE].

[87] A. Smirnov and V. Smirnov, FIRE4, LiteRed and accompanying tools to solve integration by parts relations, arXiv: 1302.5885 [INSPIRE].

[88] S. Caron-Huot and K.J. Larsen, Uniqueness of two-loop master contours, JHEP 10 (2012) 026 [arXiv: 1205.0801] [INSPIRE].

[89] N. Arkani-Hamed et al., Scattering amplitudes and the positive Grassmannian, arXiv: 1212.5605 [INSPIRE].

[90] A.E. Lipstein and L. Mason, From the holomorphic Wilson loop to 'd log' loop-integrands for super-Yang-Mills amplitudes, JHEP 05 (2013) 106 [arXiv:1212.6228] [INSPIRE]. 